\title{
Rhodium(II)-Catalyzed Annulative Coupling of $\beta$-Ketothioamides with $\alpha$-Diazo compounds: Access to Highly Functionalized Thiazolidin-4-ones and Thiazolines
}

Monish Arbaz Ansari, Dhananjay Yadav and Maya Shankar Singh*

Department of Chemistry, Institute of Science, Banaras Hindu University, Varanasi 221005, India

\section{Table of Contents}

Structure of diverse $\beta$-ketothioamides utilized for thiazoilidinones and thiazolines synthesis

(Table S1)

NMR Spectra of Compounds

Thermal ellipsoid plot and crystallization detail of compound $\mathbf{3 e}$

ORTEP diagram of compound 3e

The positive-ion ESI mass spectrum of reaction mixture

(Showing existence of $N, S$-acetal intermediate $\mathbf{C}$ ) 
Table S1. Structure of diverse $\beta$-ketothioamides utilized for thiazoilidinones and thiazolines synthesis

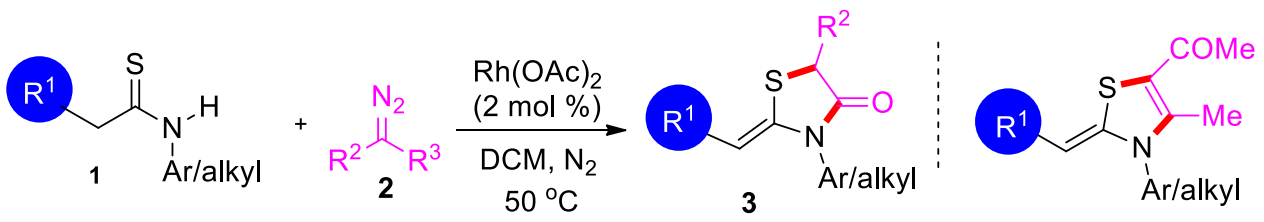

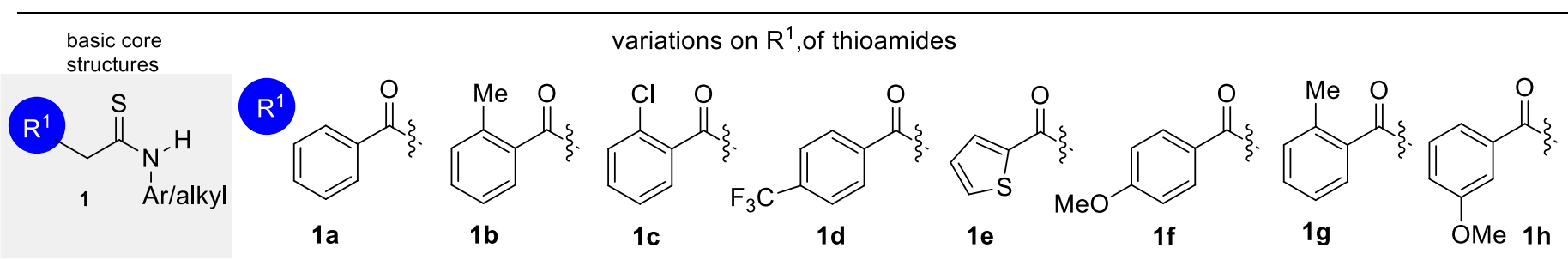<smiles>Cc1ccc(C(=O)C(=O)c2ccc(C(=O)C(=O)c3ccc(C(=O)c4ccc(Br)cc4)cc3)cc2)cc1</smiles>

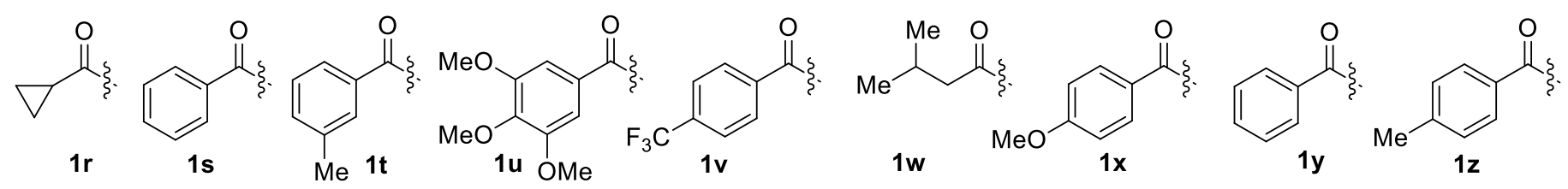

Note: $1 \mathrm{a}=1 \mathrm{~s}=1 \mathrm{y}, 1 \mathrm{~b}=1 \mathrm{~g}, 1 \mathrm{~d}=1 \mathrm{v}, 1 \mathrm{f}=1 \mathrm{x}, 1 \mathrm{~m}=1 \mathrm{q}=1 \mathrm{z}$ 
NMR Spectra of the compounds (3a-3ec and 4a-4b)

${ }^{1} \mathrm{H}$ and ${ }^{1} \mathrm{H}$ decoupled ${ }^{13} \mathrm{C}$ NMR spectra of compound 3a

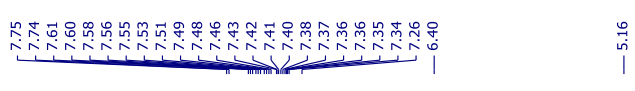

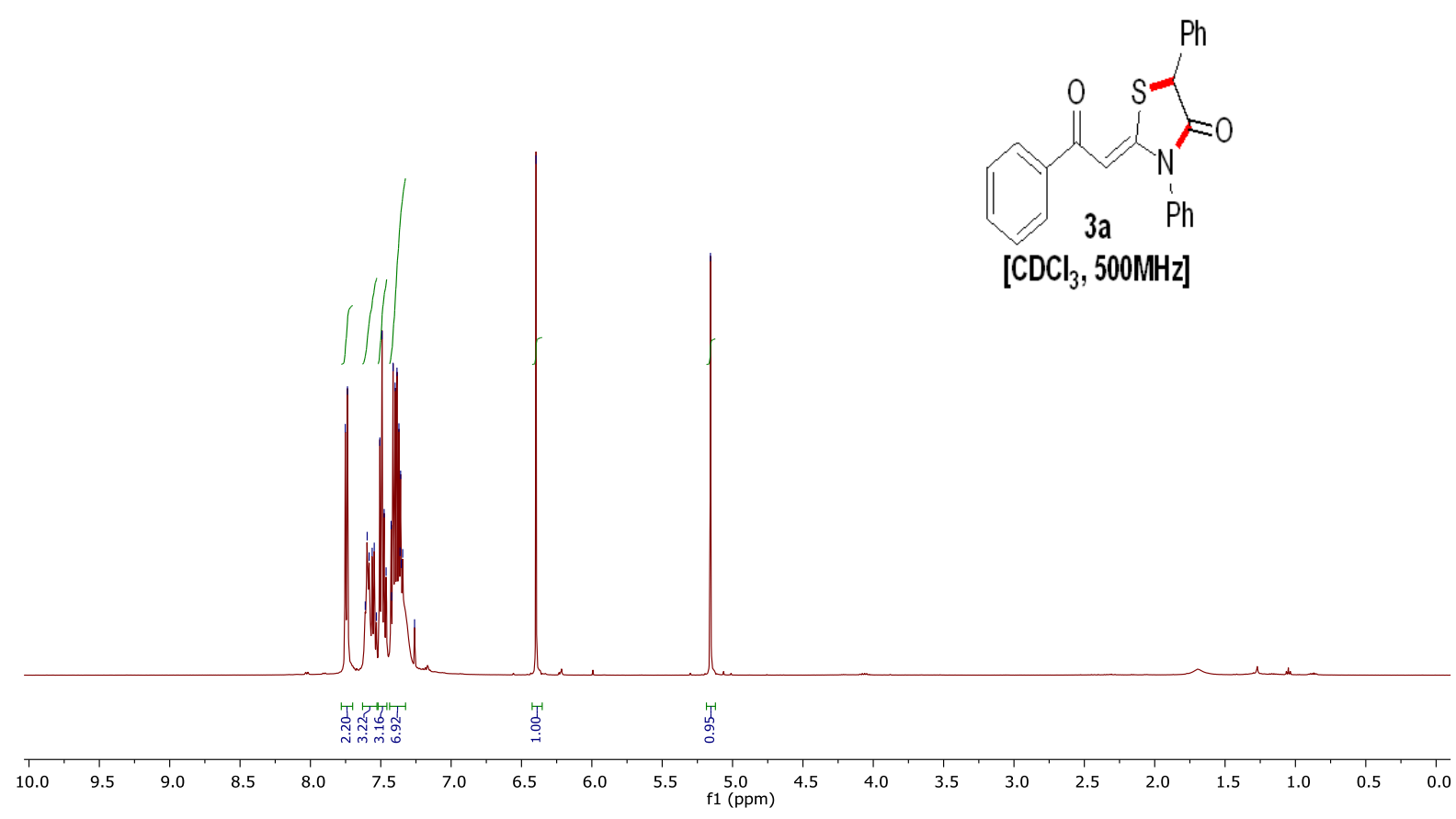

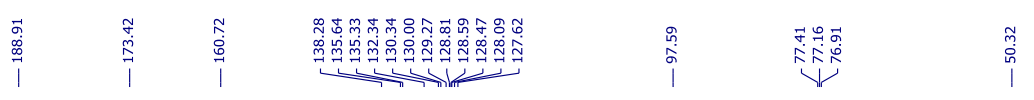<smiles>O=C(/C=C1\SC(c2ccccc2)C(=O)N1c1ccccc1)c1ccccc1</smiles>

$\left[\mathrm{CDCl}_{3}, 125 \mathrm{MHz}\right]$

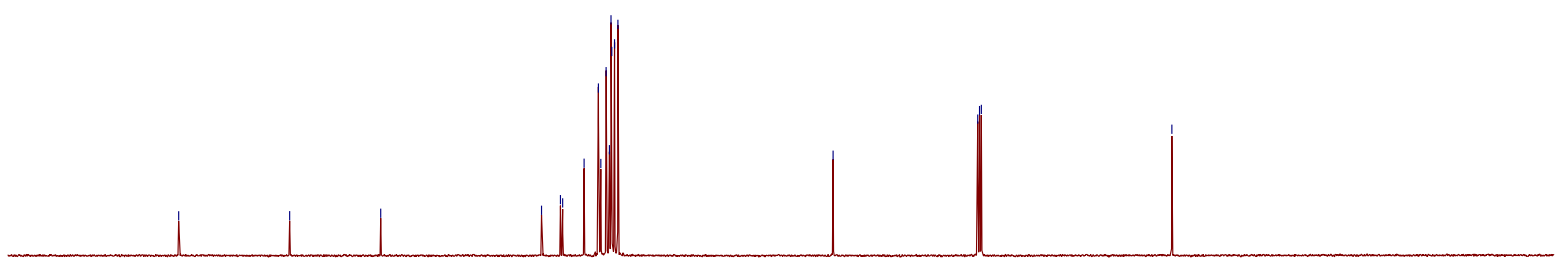

$\begin{array}{lllllllllll}210 & 200 & 190 & 180 & 170 & 160 & 150 & 140 & 130 & 120 & \begin{array}{l}110 \\ \mathrm{f} 1(\mathrm{ppm})\end{array}\end{array}$ 
${ }^{1} \mathrm{H}$ and ${ }^{1} \mathrm{H}$ decoupled ${ }^{13} \mathrm{C}$ NMR spectra of compound $\mathbf{3 b}$

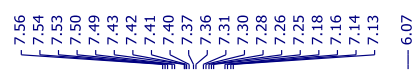

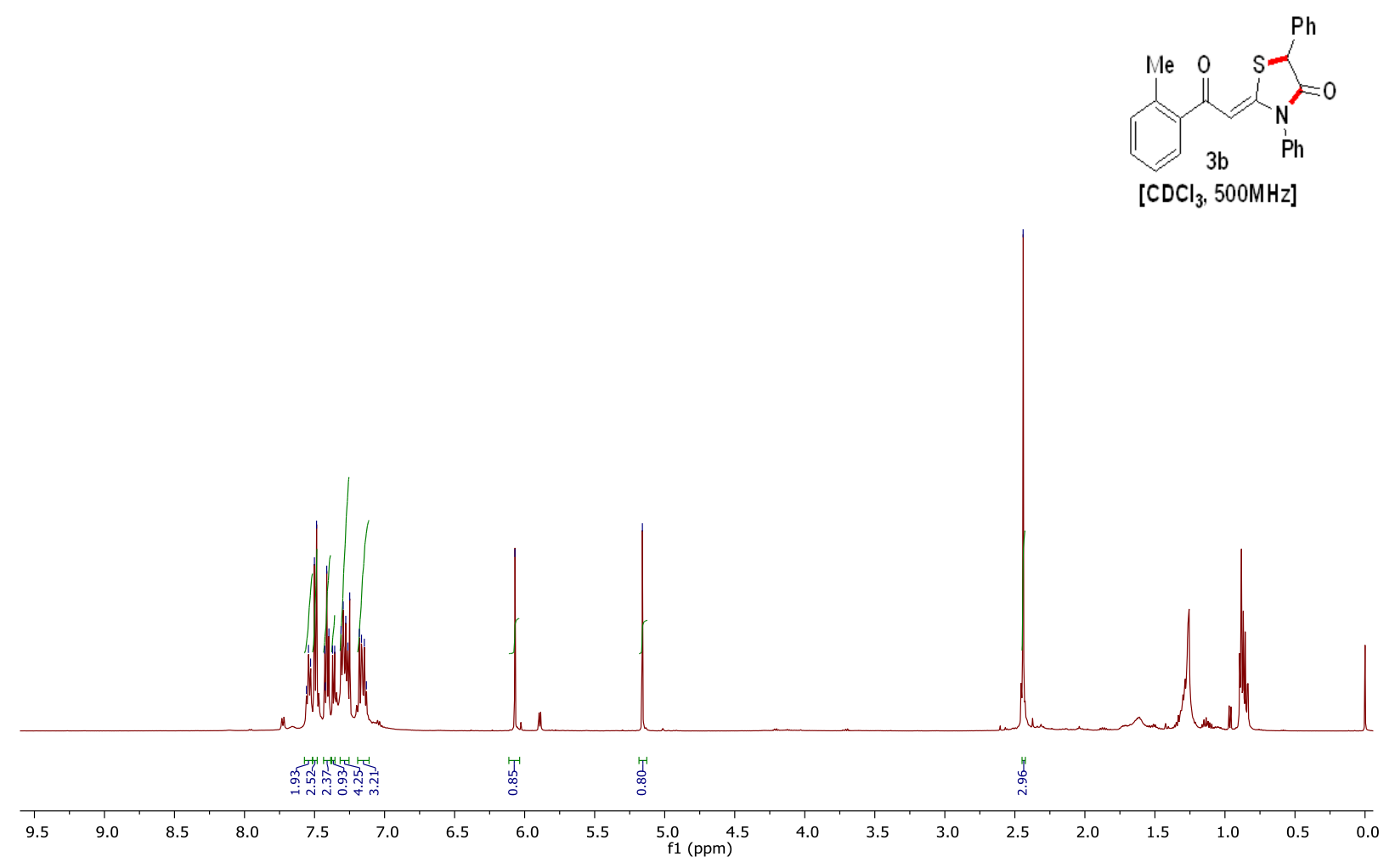

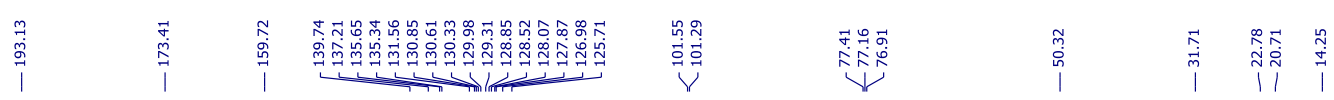

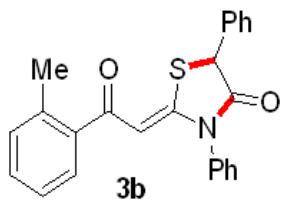

[CDCl $3,125 \mathrm{MHz}]$

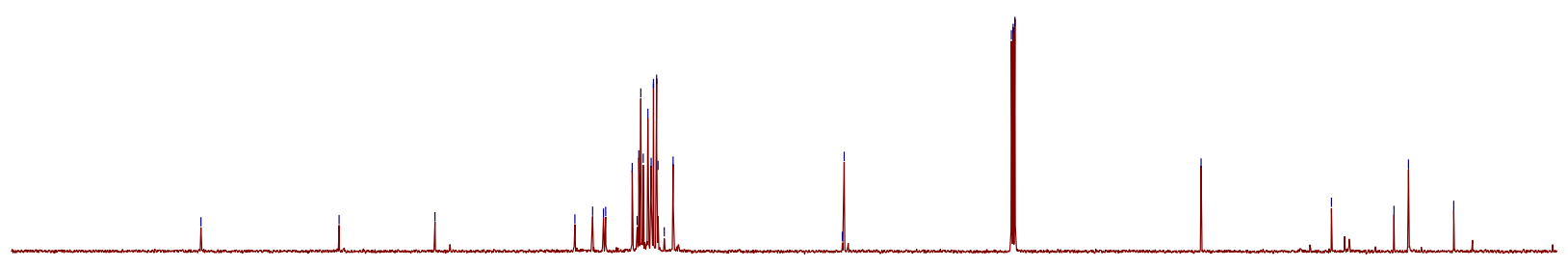

$\begin{array}{lllllllllllllllllllllllllllllllllll}220 & 210 & 200 & 190 & 180 & 170 & 160 & 150 & 140 & 130 & 120 & 110 & 100 & 90 & 80 & 70 & 60 & 50 & 40 & 30 & 20 & 10 & 0\end{array}$ 
${ }^{1} \mathrm{H}$ and ${ }^{1} \mathrm{H}$ decoupled ${ }^{13} \mathrm{C}$ NMR spectra of compound $\mathbf{3 c}$

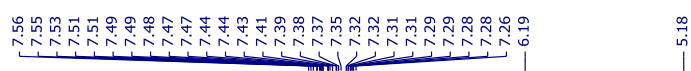

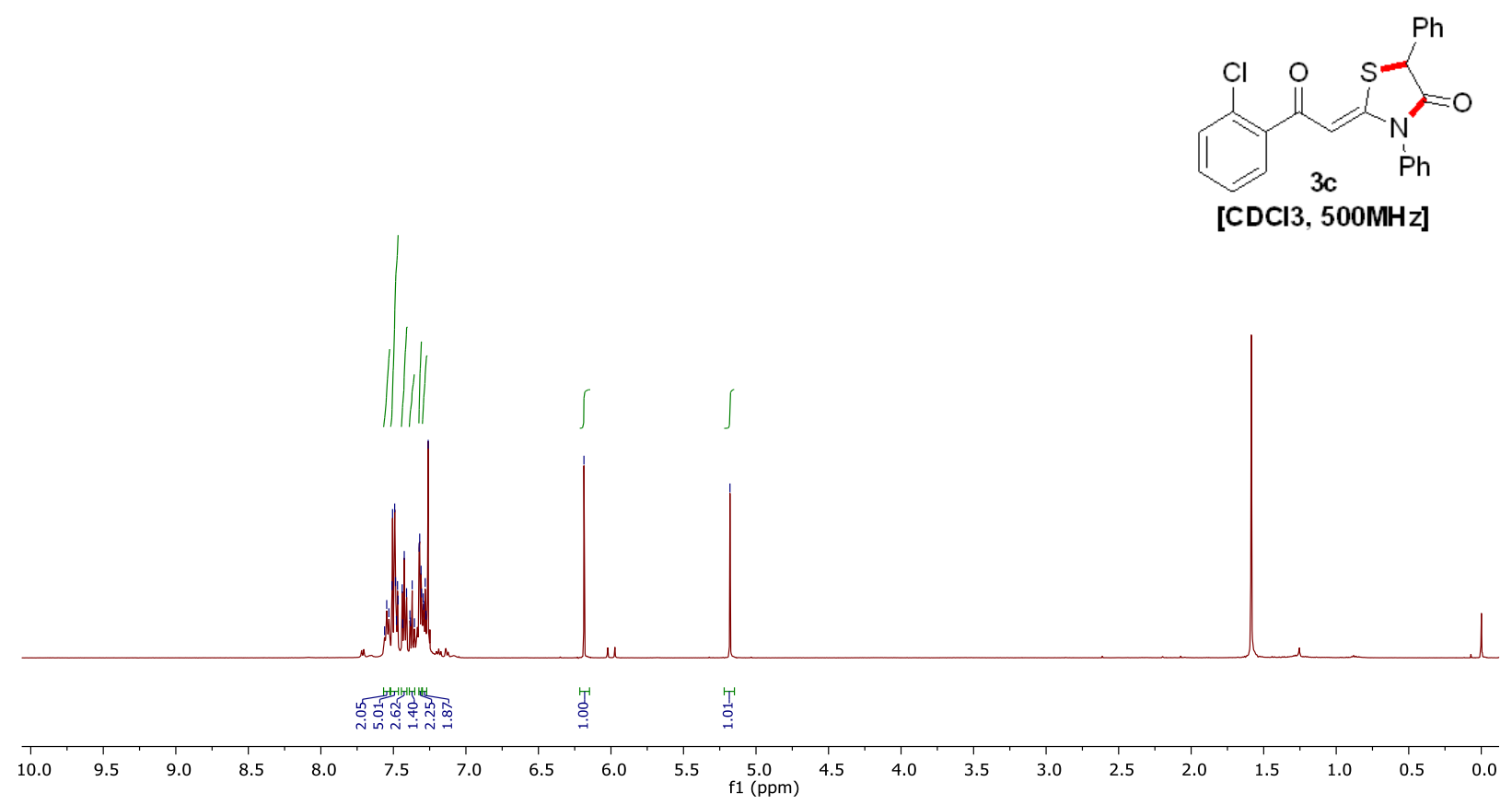

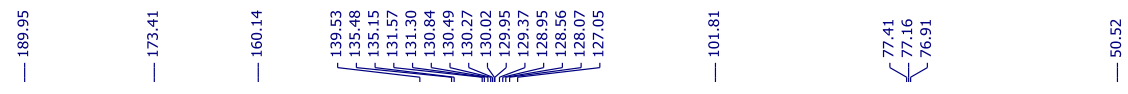

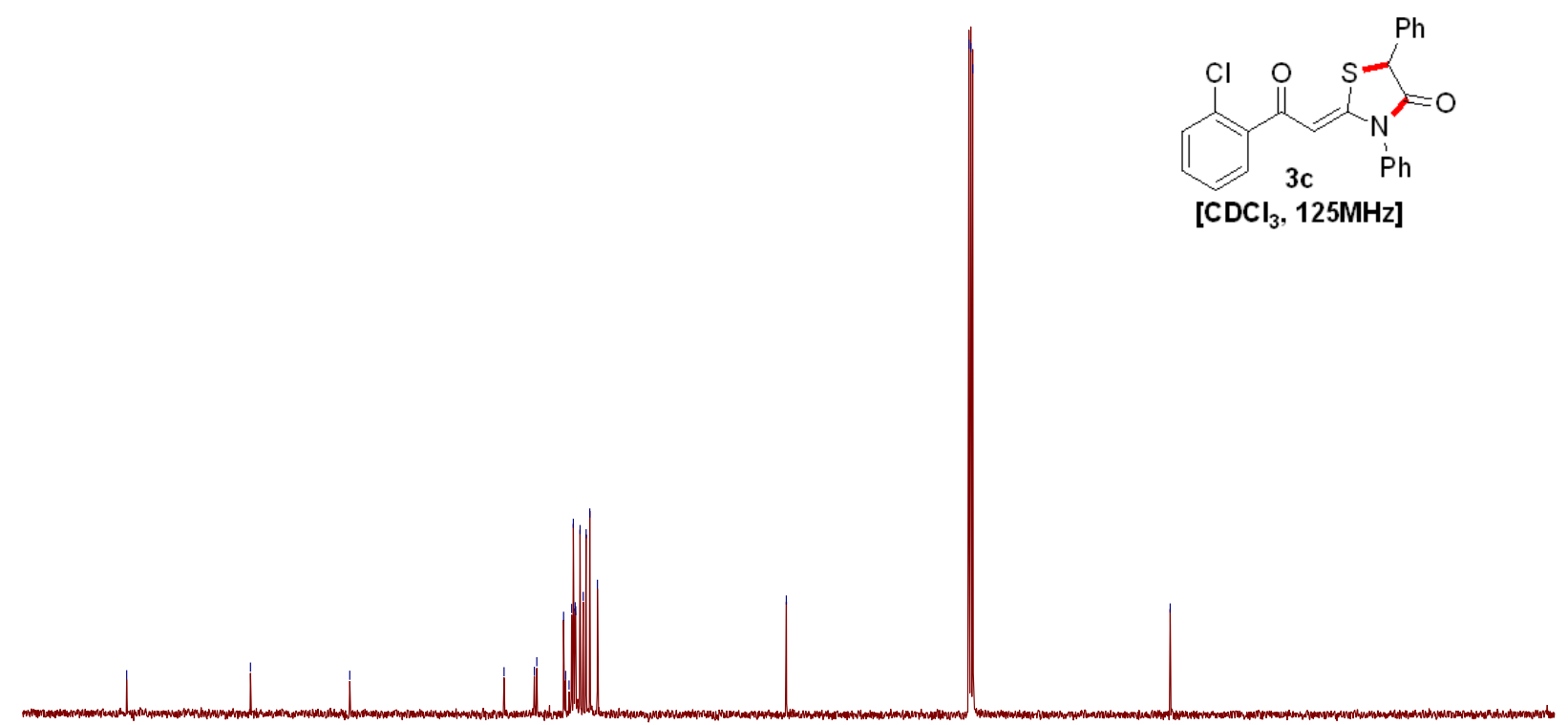

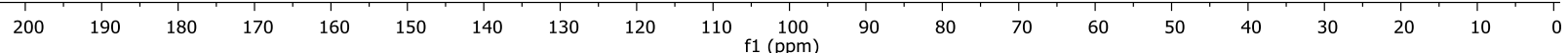


${ }^{1} \mathrm{H}$ and ${ }^{1} \mathrm{H}$ decoupled ${ }^{13} \mathrm{C}$ NMR spectra of compound 3d

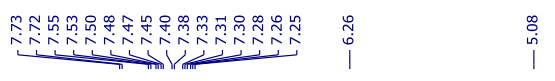
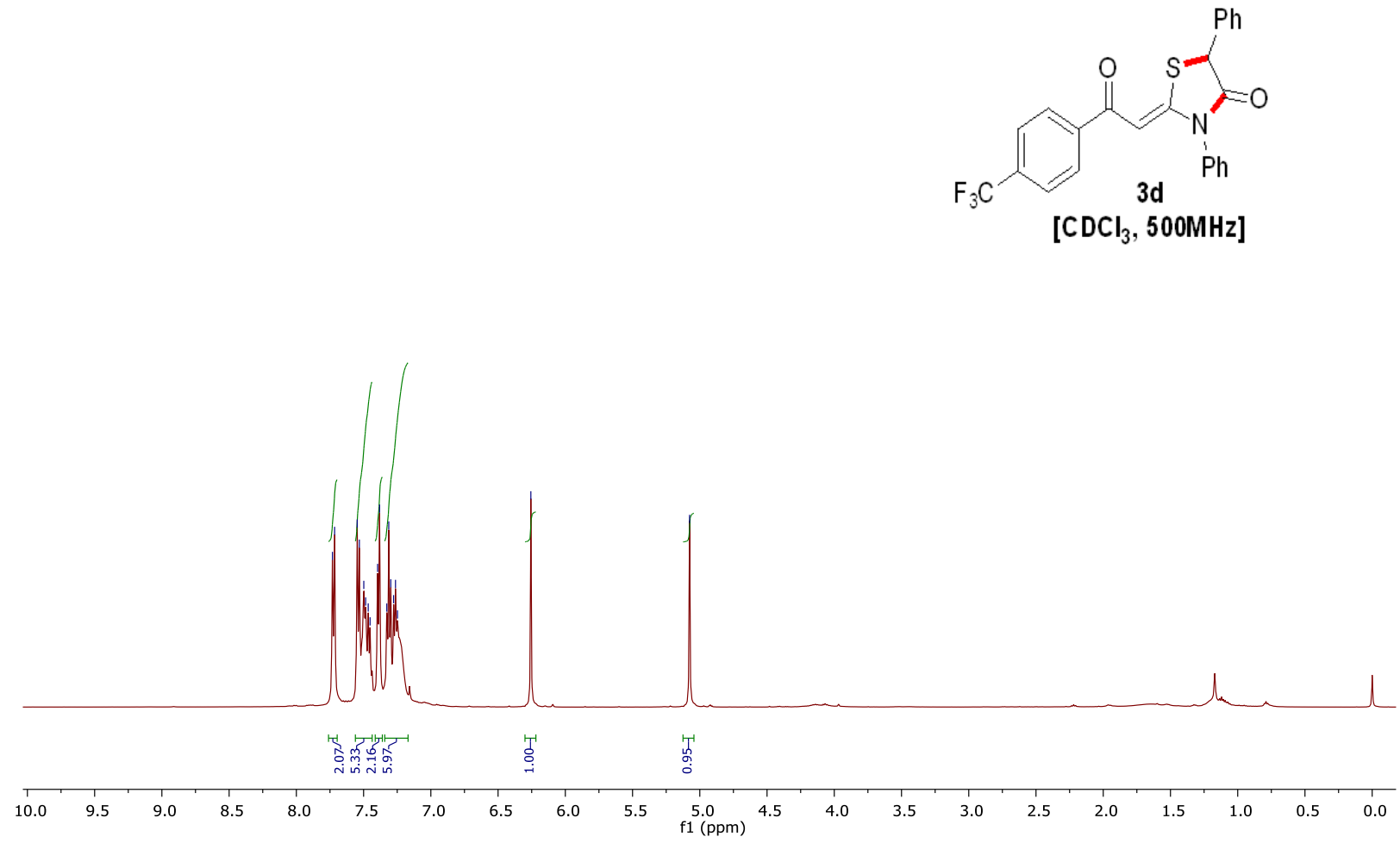

$\stackrel{\substack{n \\ 0}}{\substack{n \\ \infty}}$

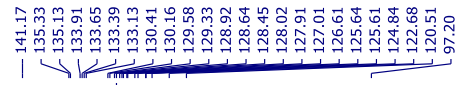

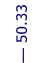

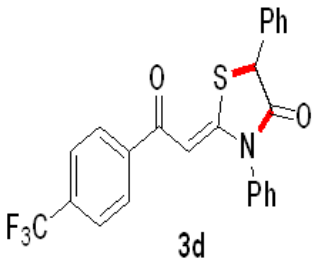

[CDCl $3,125 \mathrm{MHz}]$

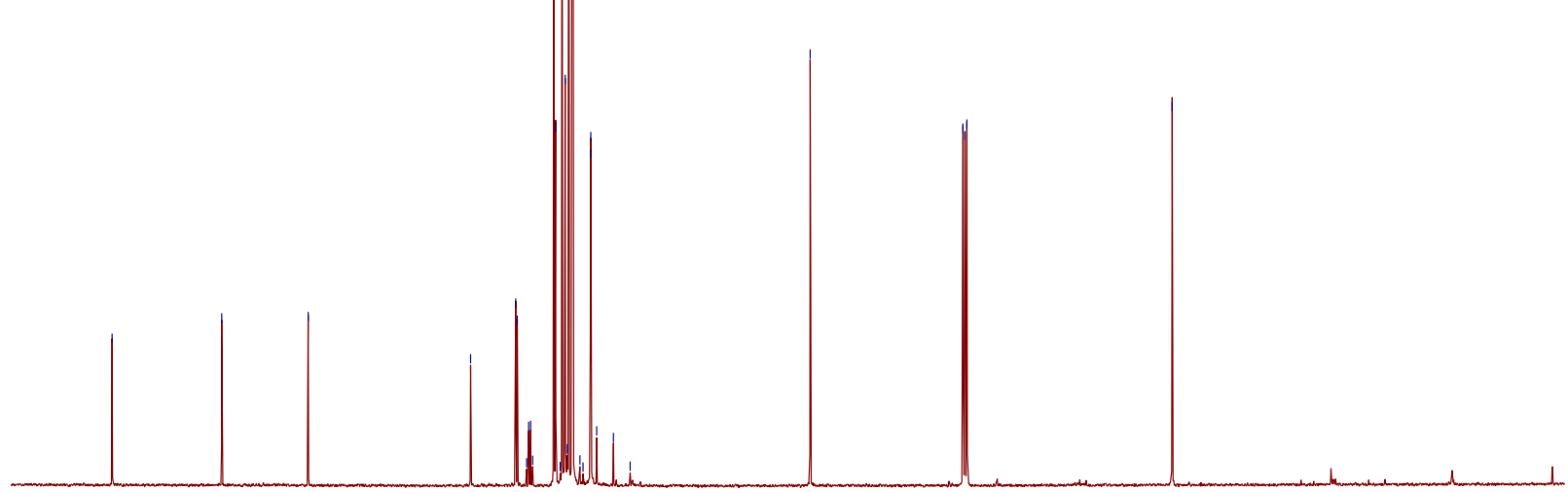

200
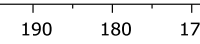

$160 \quad 150$

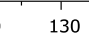

$\begin{array}{lll}120 & 110 & 100 \\ f 1(\mathrm{ppm})\end{array}$ 
${ }^{1} \mathrm{H}$ and ${ }^{1} \mathrm{H}$ decoupled ${ }^{13} \mathrm{C}$ NMR spectra of compound $\mathbf{3 e}$

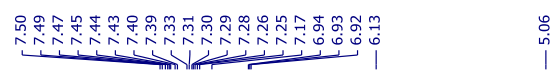

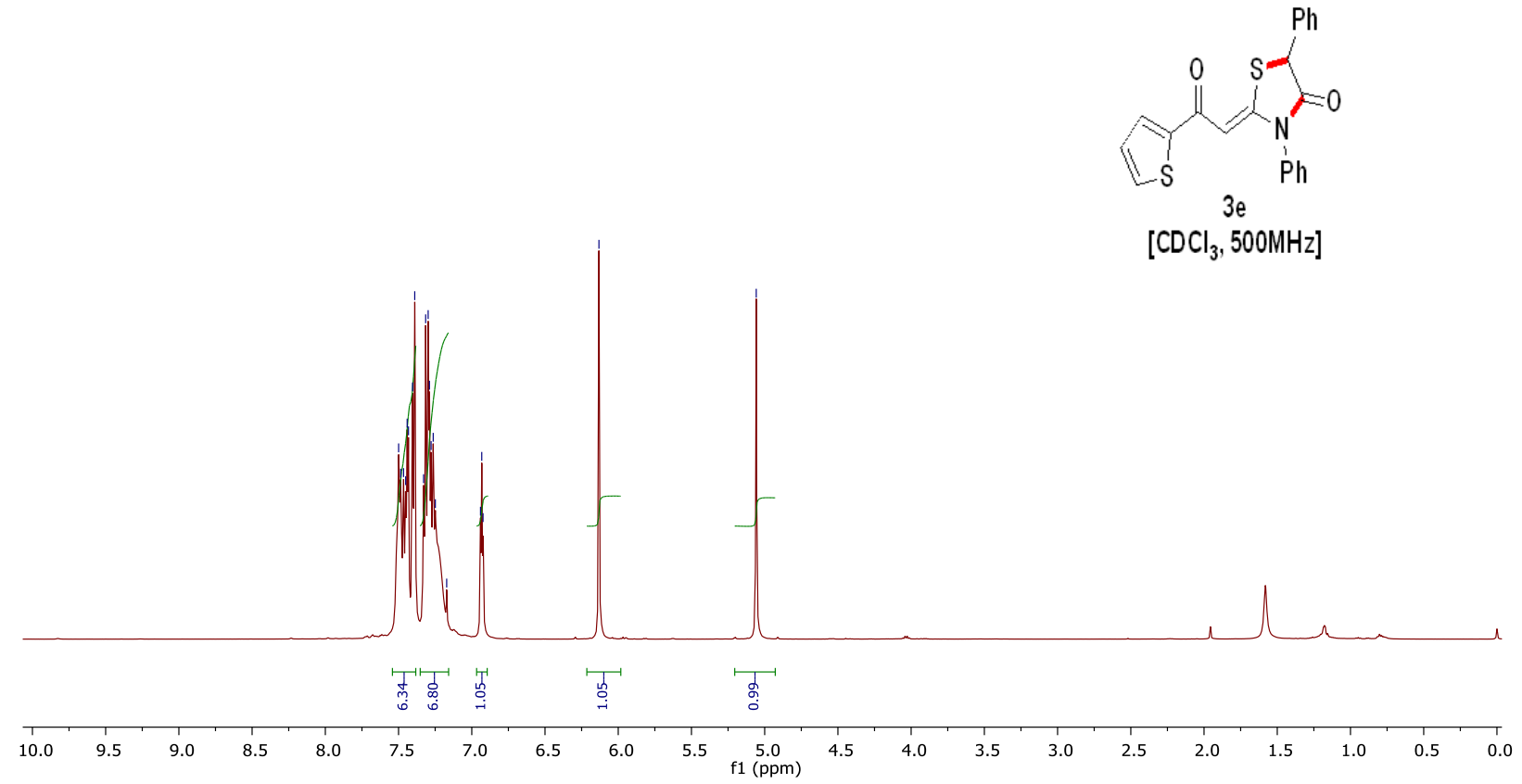

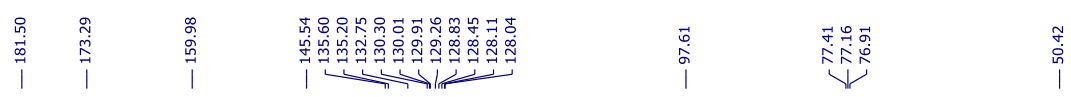

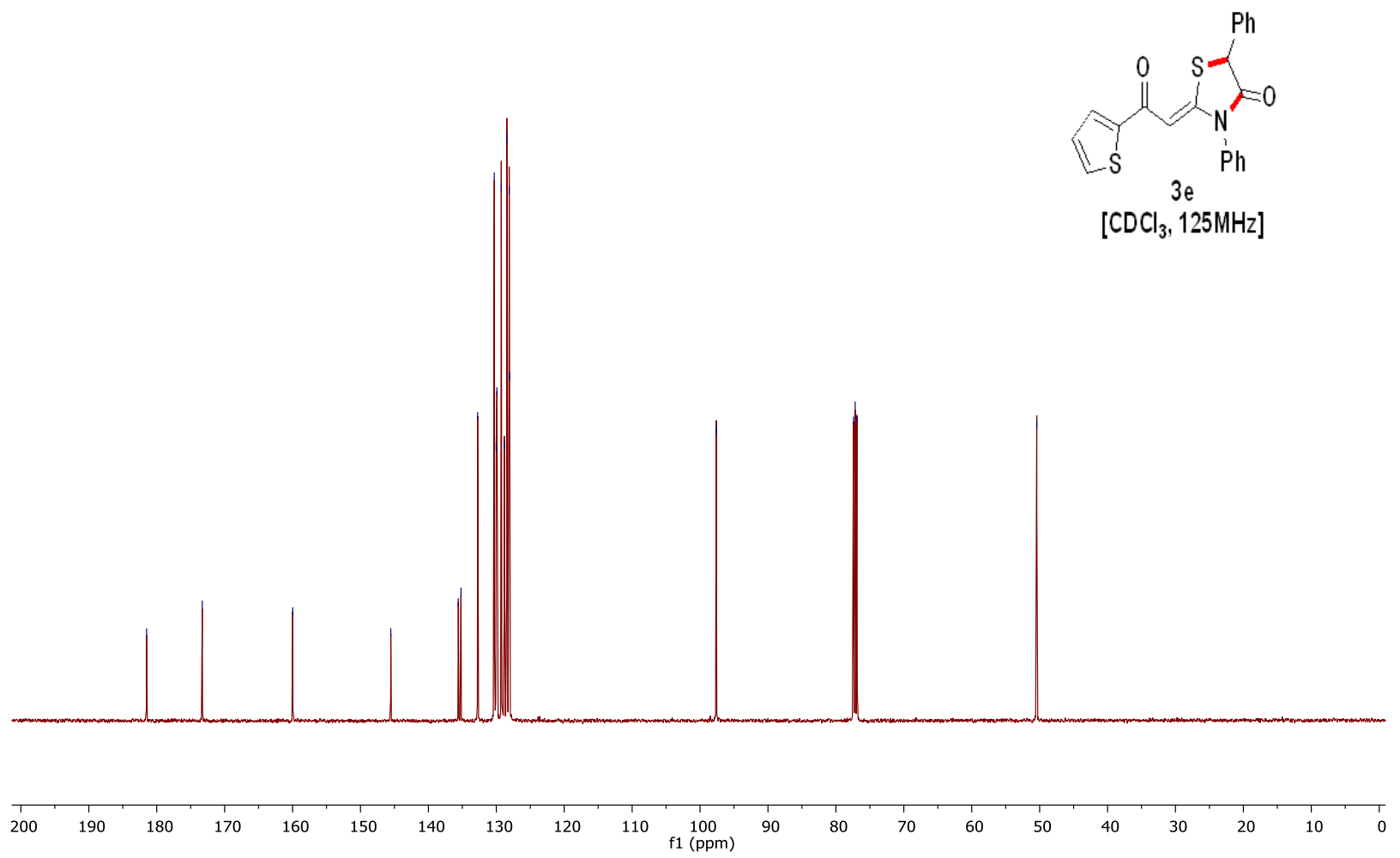


${ }^{1} \mathrm{H}$ and ${ }^{1} \mathrm{H}$ decoupled ${ }^{13} \mathrm{C}$ NMR spectra of compound $\mathbf{3 f}$

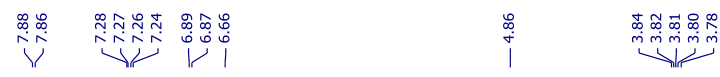

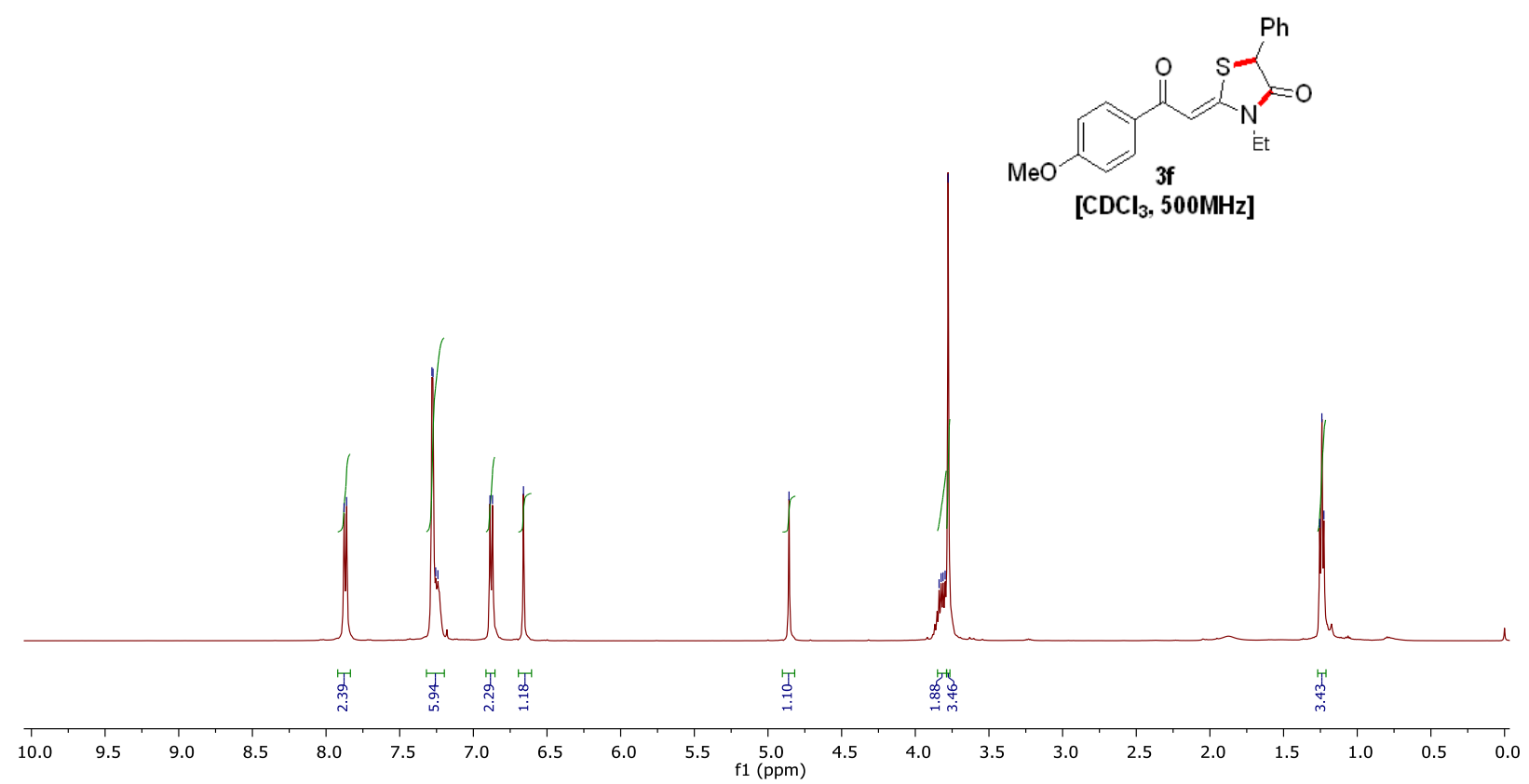

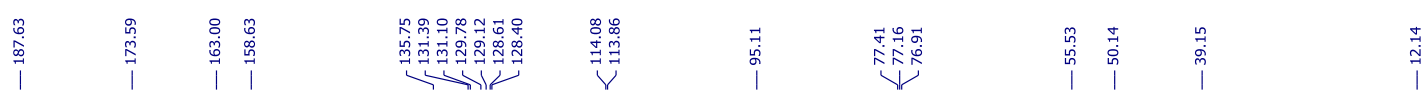

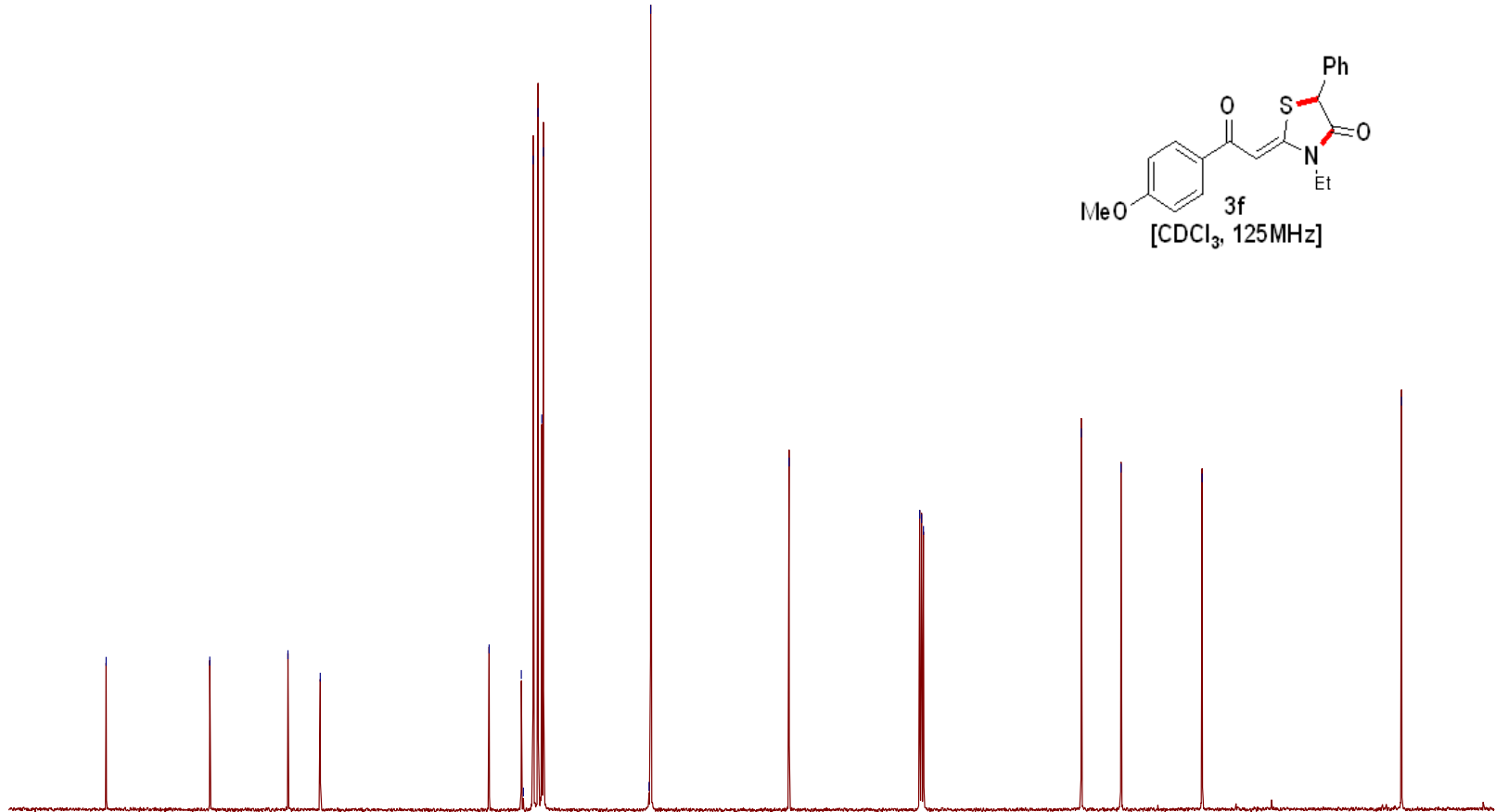

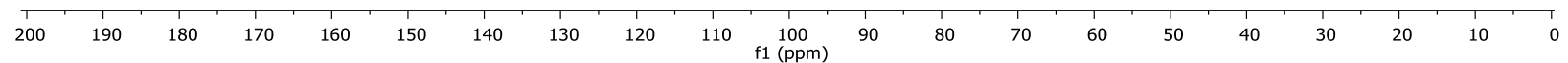


${ }^{1} \mathrm{H}$ and ${ }^{1} \mathrm{H}$ decoupled ${ }^{13} \mathrm{C}$ NMR spectra of compound $\mathbf{3 g}$

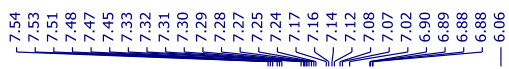

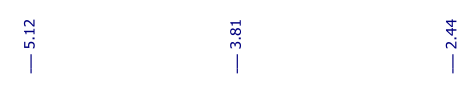
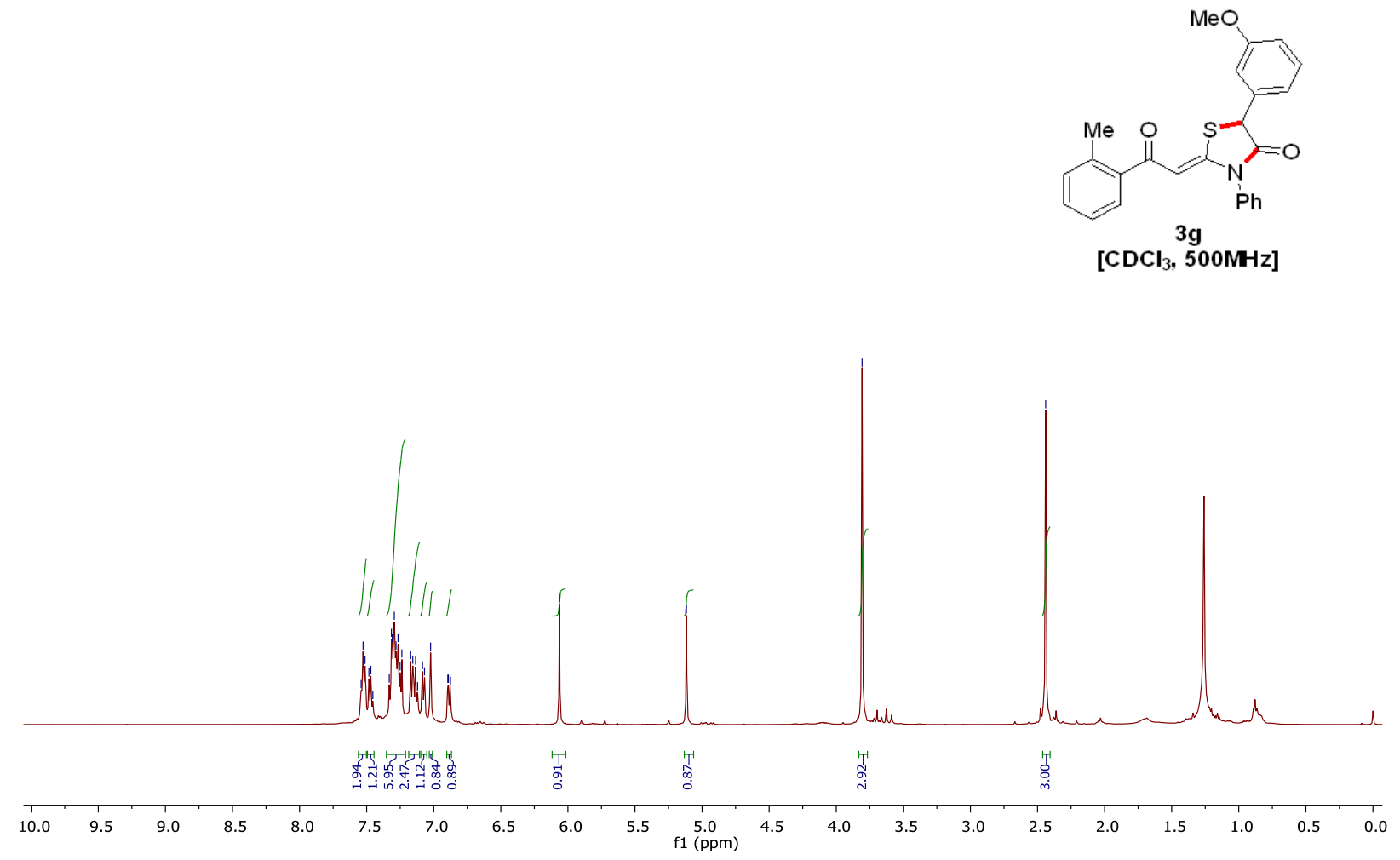

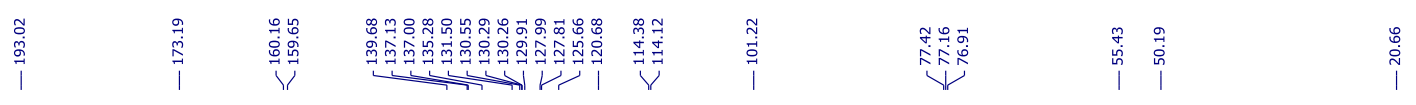

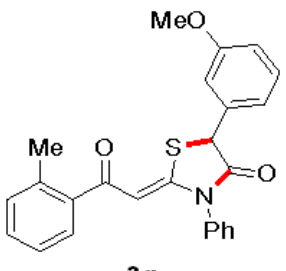

$\left[\mathrm{CDCl}_{3}, 125 \mathrm{MHz}\right]$

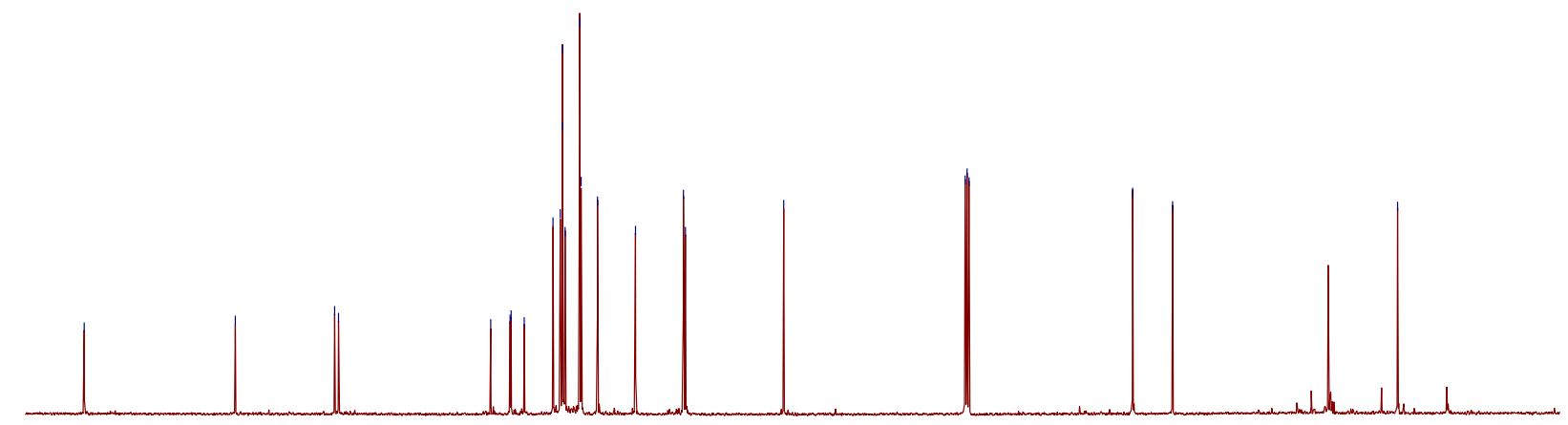

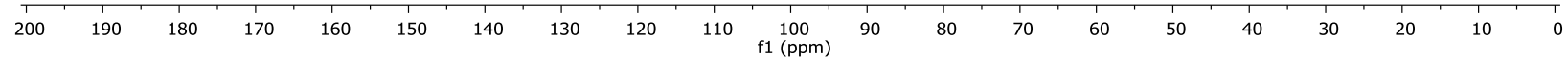


${ }^{1} \mathrm{H}$ and ${ }^{1} \mathrm{H}$ decoupled ${ }^{13} \mathrm{C}$ NMR spectra of compound $\mathbf{3 h}$

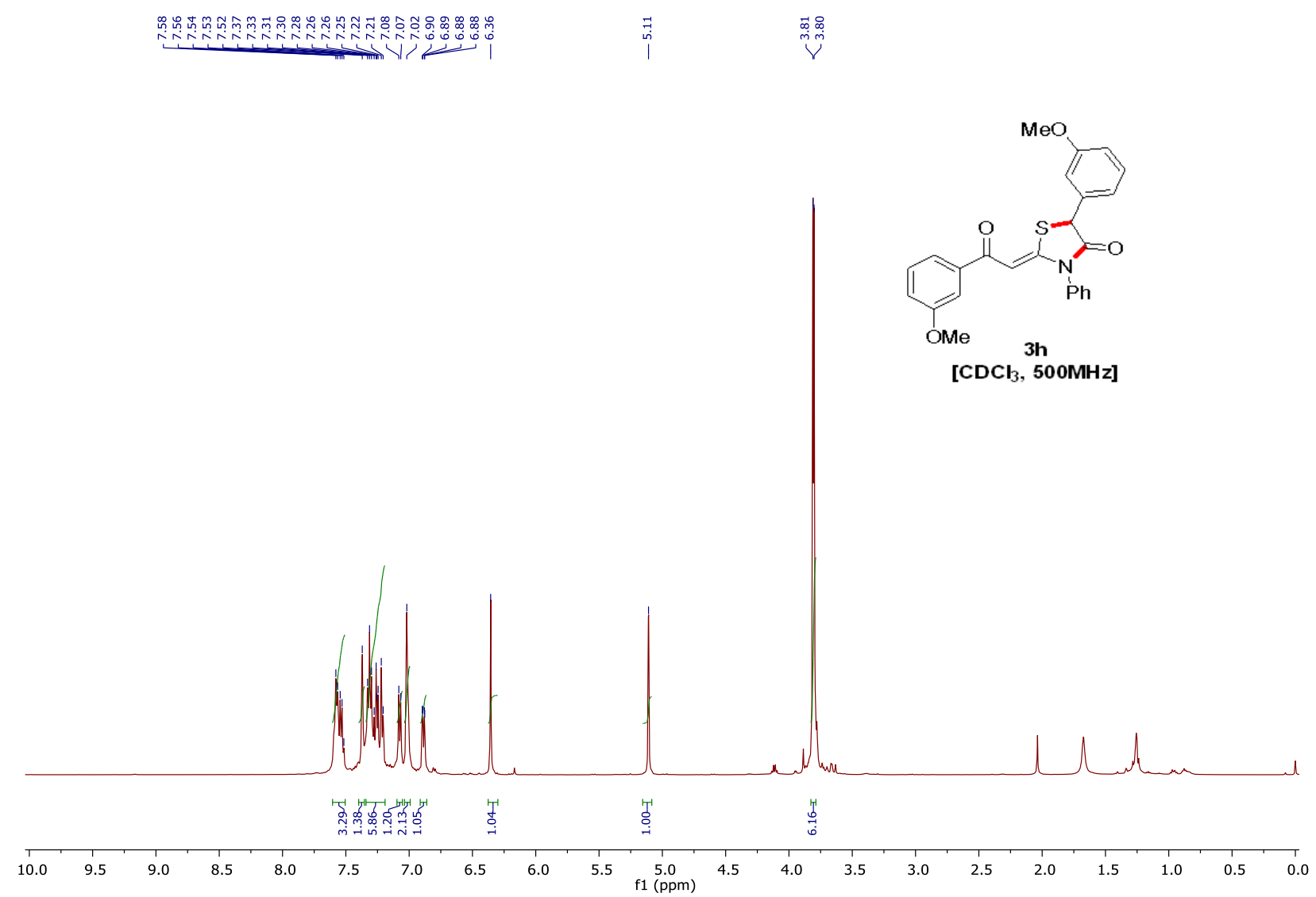

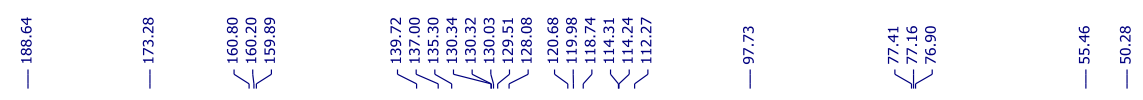

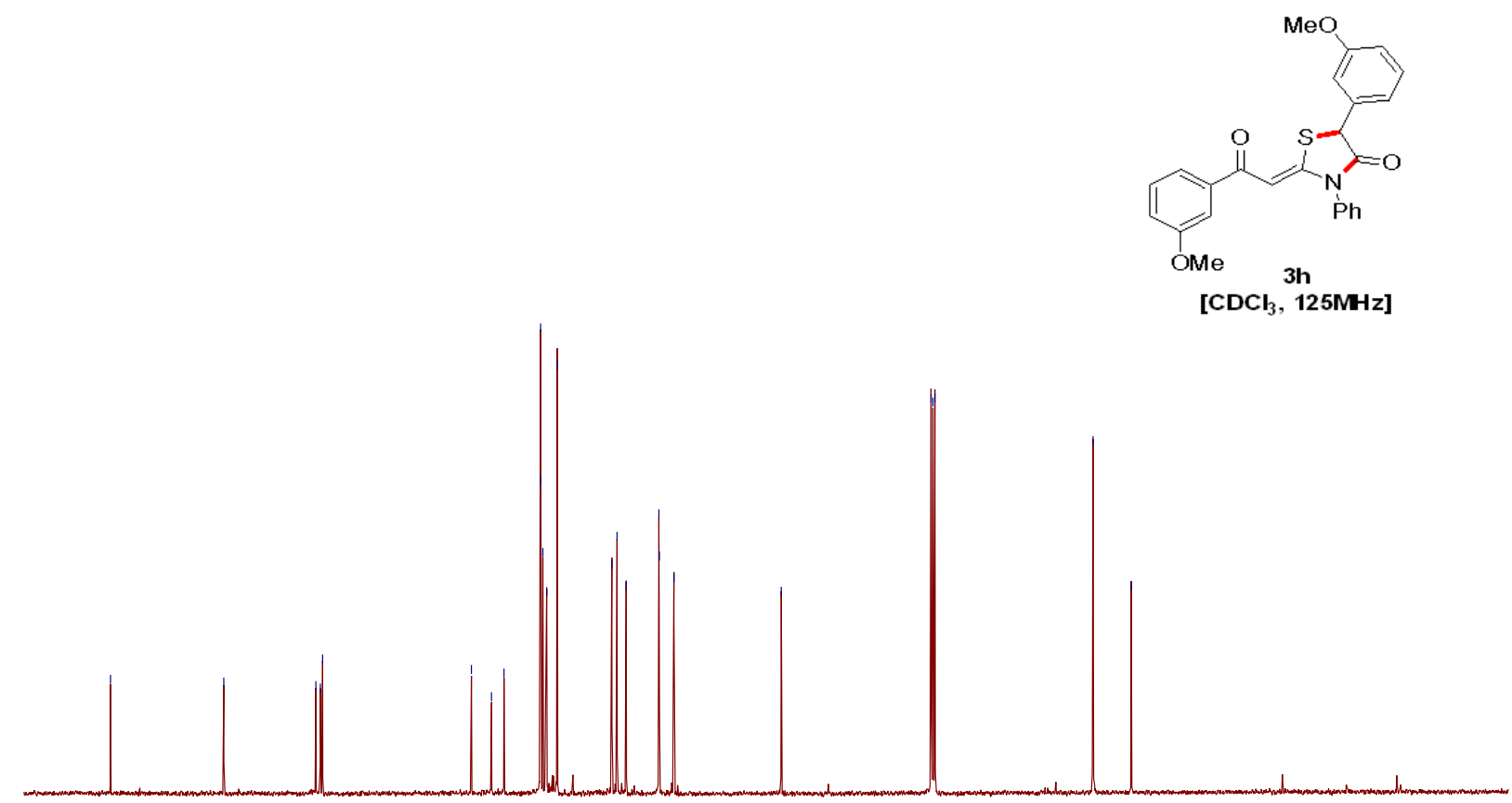

200
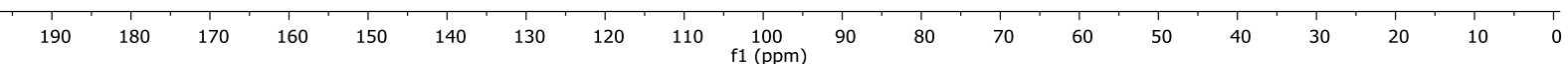
${ }^{1} \mathrm{H}$ and ${ }^{1} \mathrm{H}$ decoupled ${ }^{13} \mathrm{C}$ NMR spectra of compound $\mathbf{3 i}$

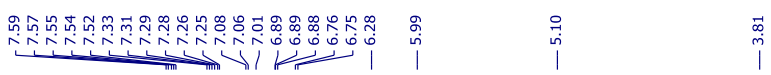

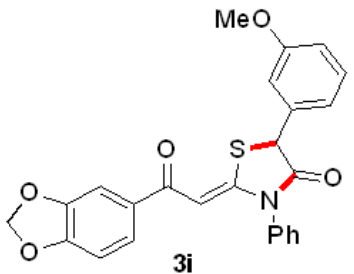

[ $\left.\mathrm{CDCl}_{3}, 500 \mathrm{MHz}\right]$

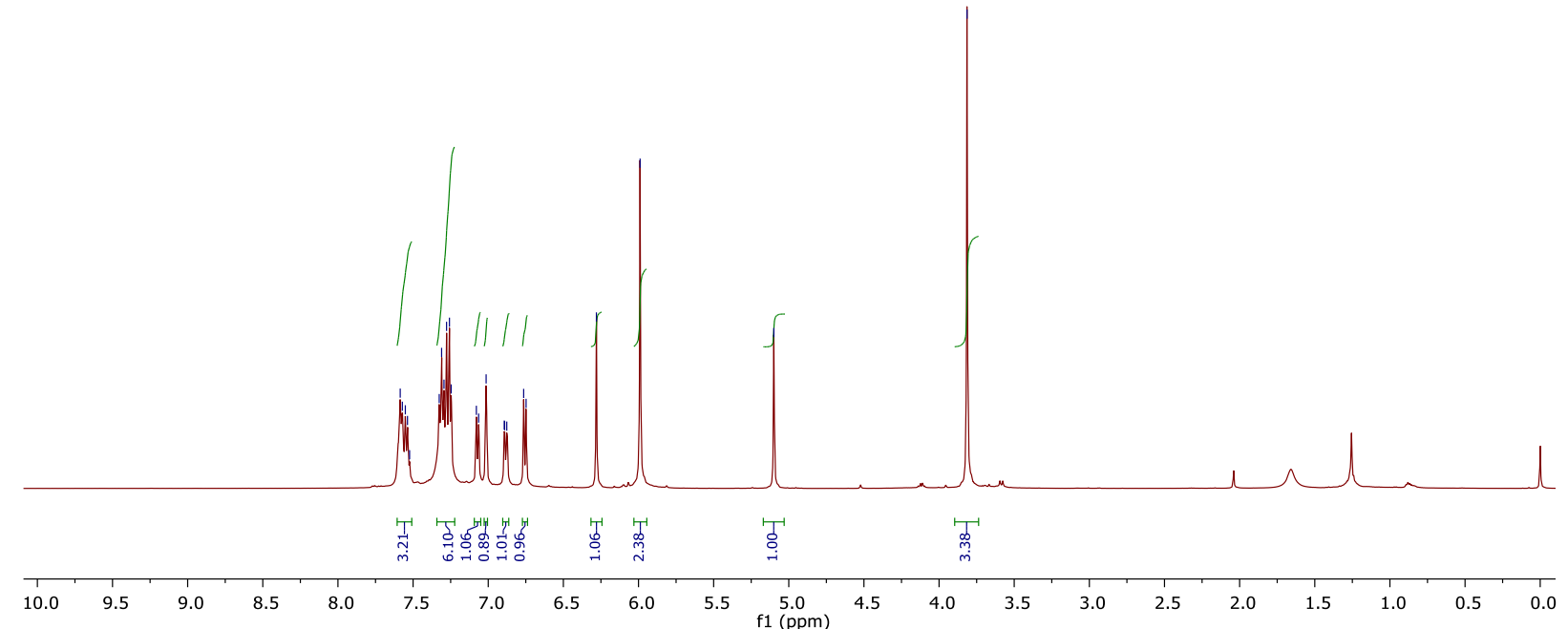

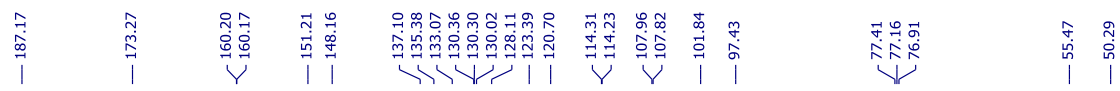

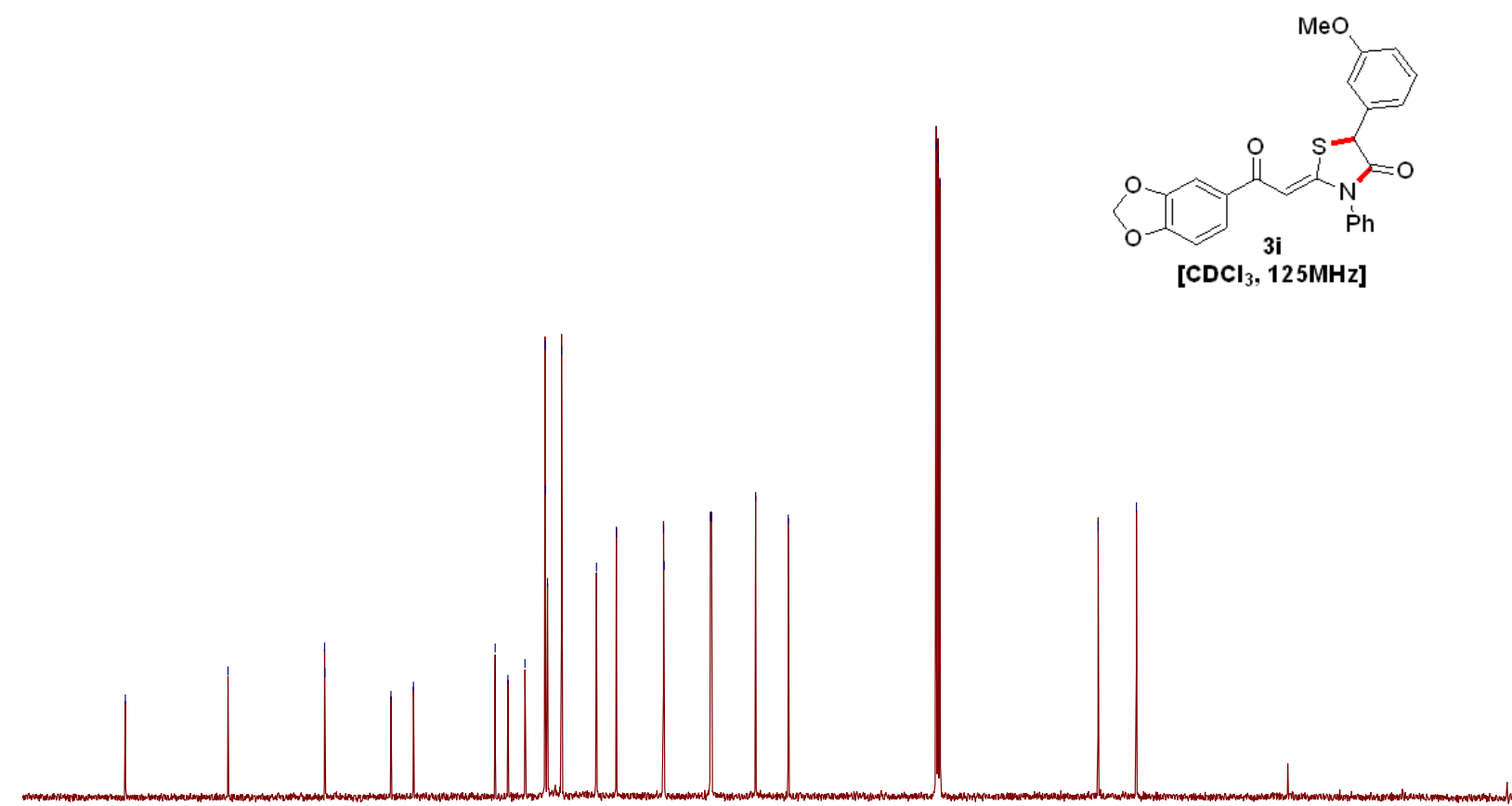

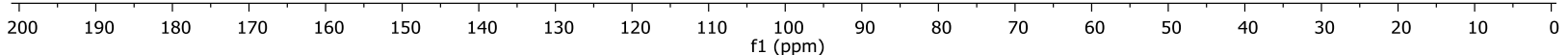


${ }^{1} \mathrm{H}$ and ${ }^{1} \mathrm{H}$ decoupled ${ }^{13} \mathrm{C}$ NMR spectra of compound $\mathbf{3 j}$

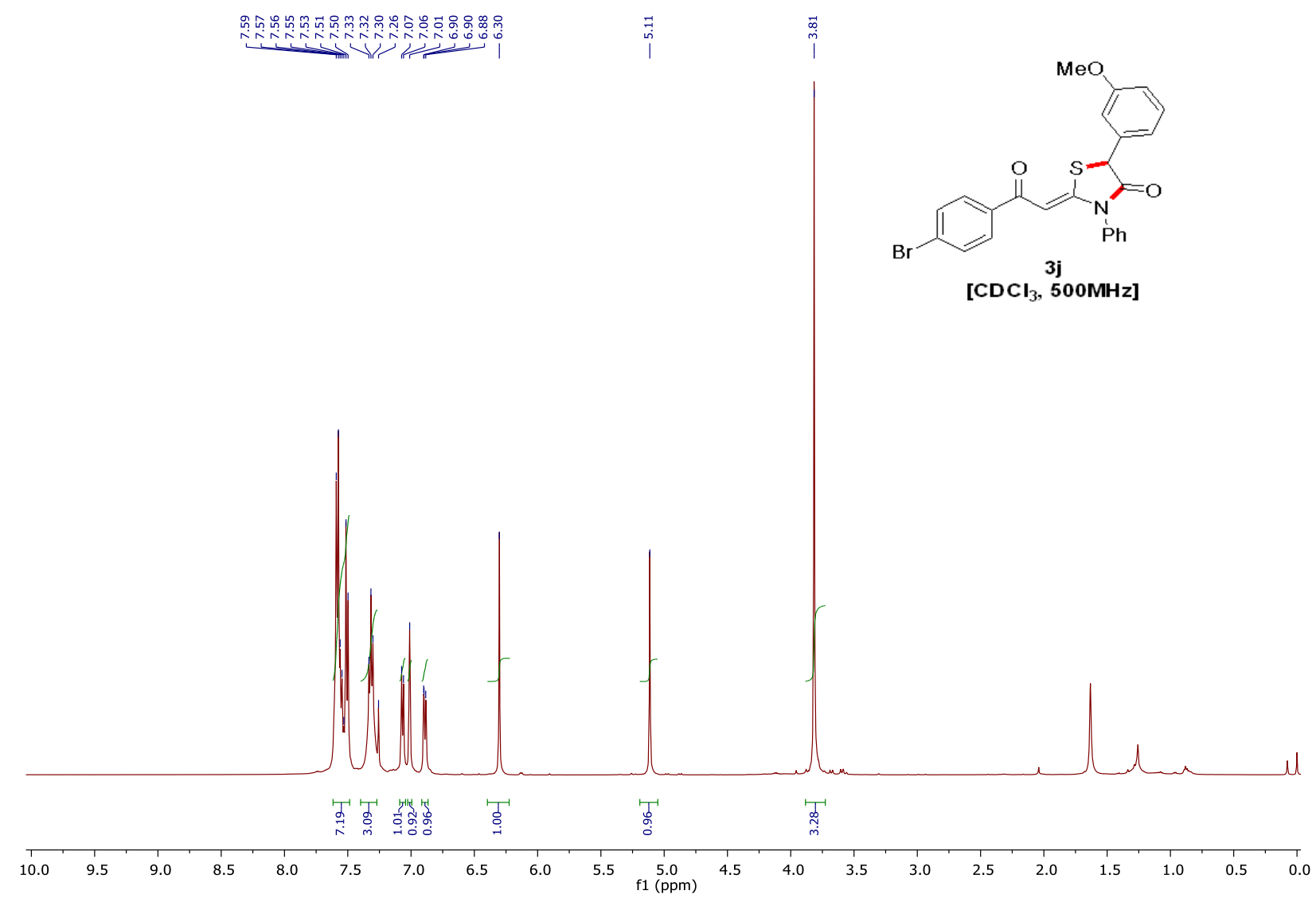

\begin{tabular}{|c|c|c|c|c|c|c|c|}
\hline & 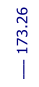 & 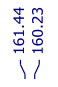 & 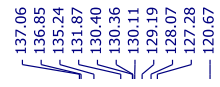 & 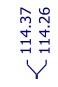 & 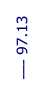 & 说 & \\
\hline
\end{tabular}

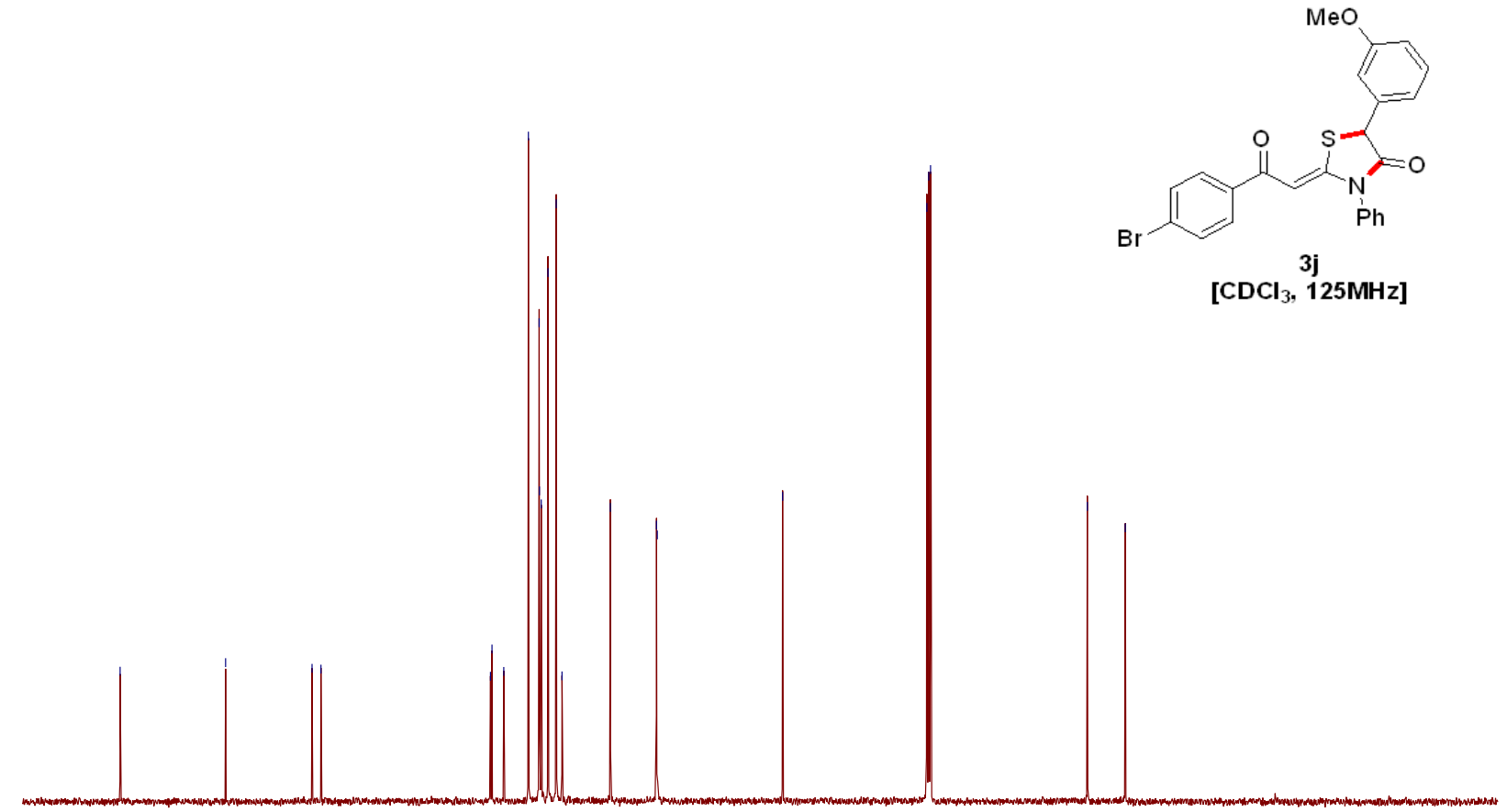

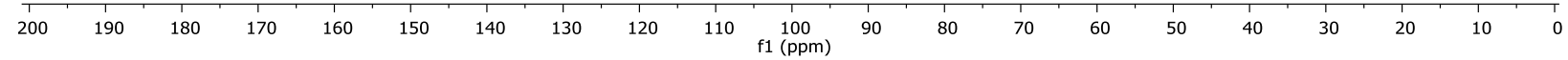


${ }^{1} \mathrm{H}$ and ${ }^{1} \mathrm{H}$ decoupled ${ }^{13} \mathrm{C}$ NMR spectra of compound 3k

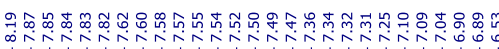

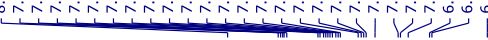

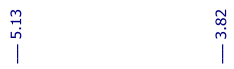

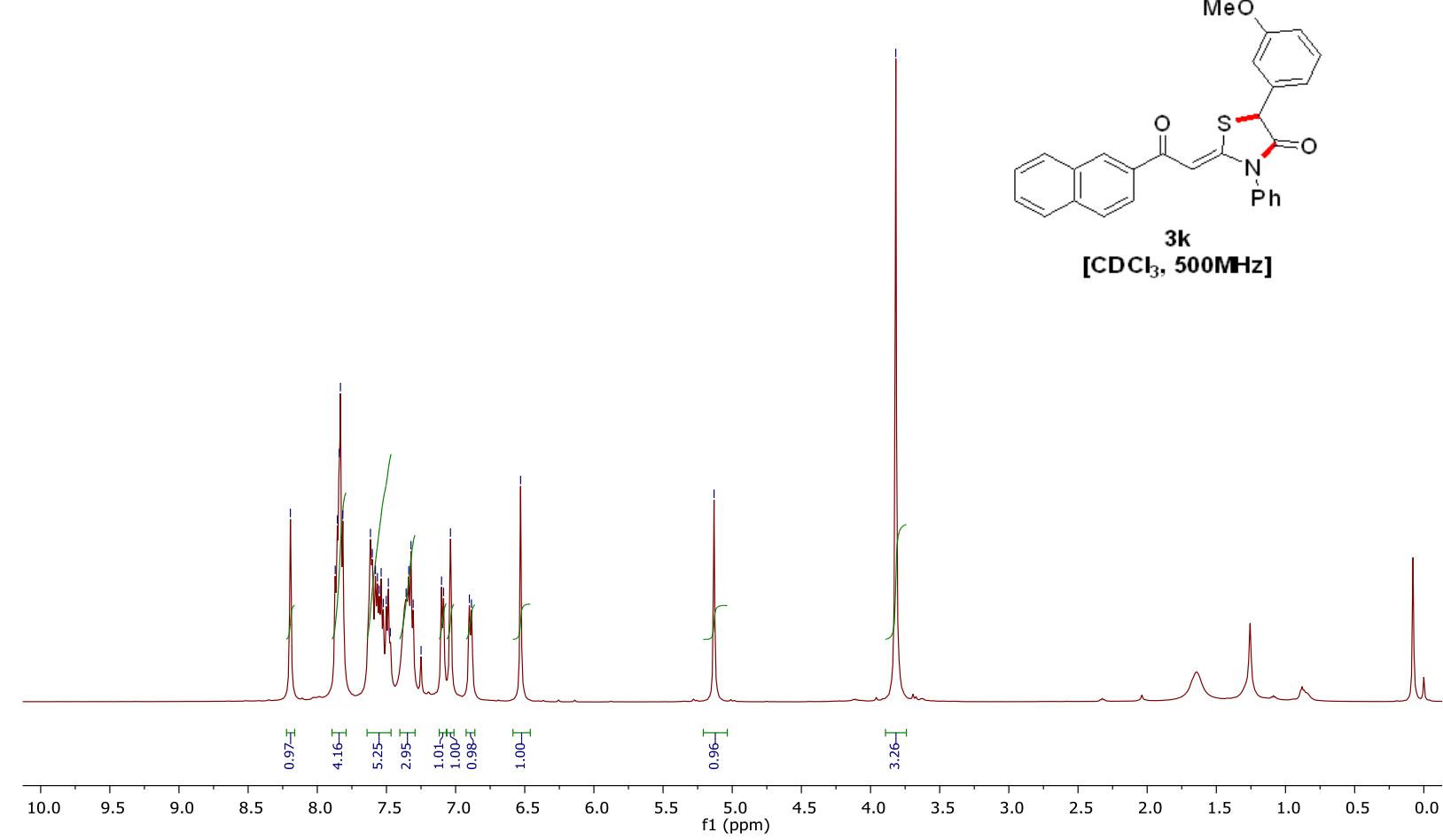

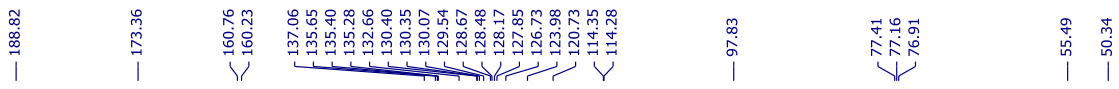
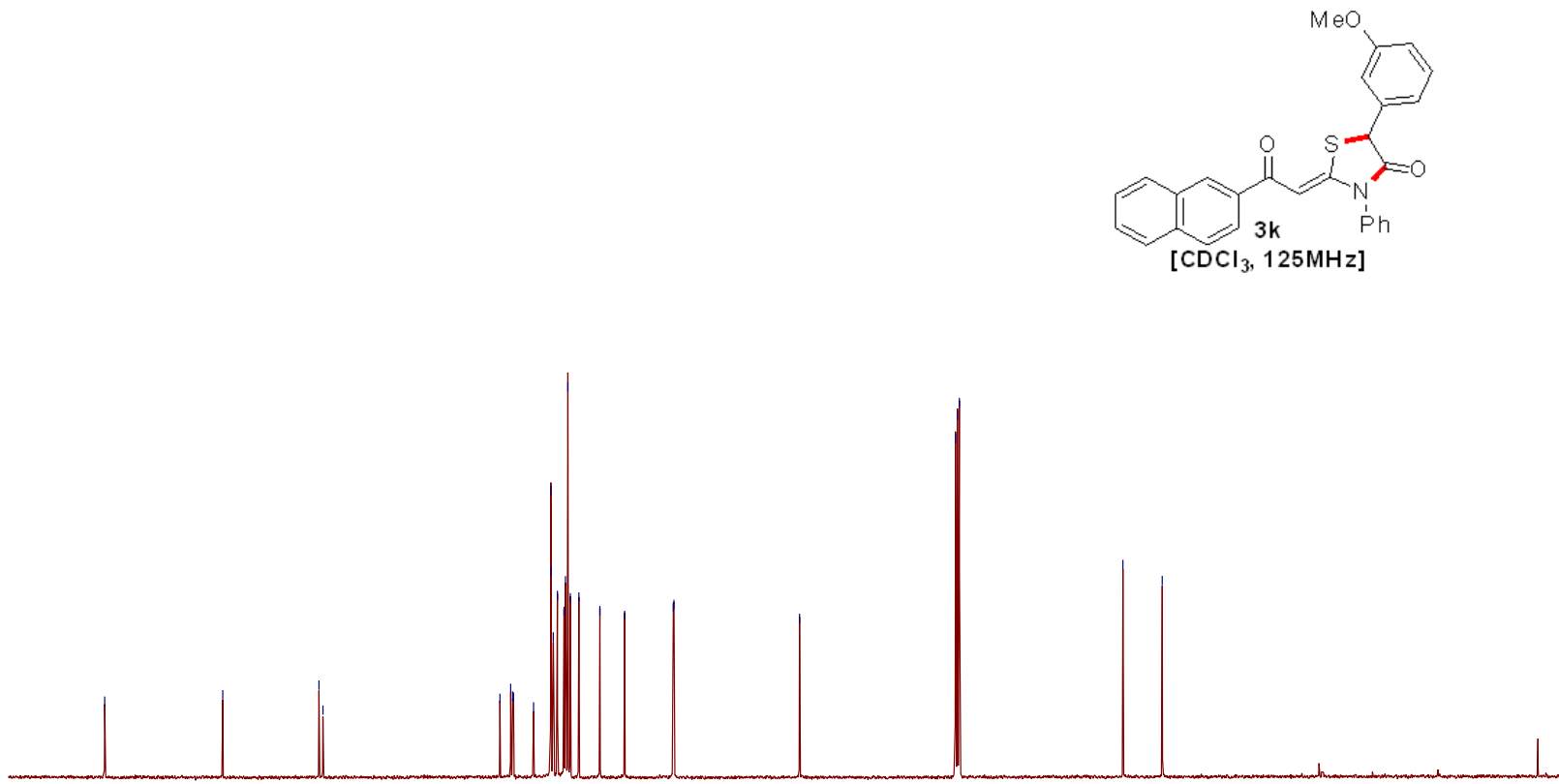

200

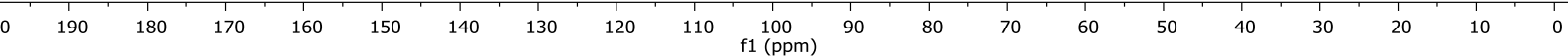


${ }^{1} \mathrm{H}$ and ${ }^{1} \mathrm{H}$ decoupled ${ }^{13} \mathrm{C}$ NMR spectra of compound 31

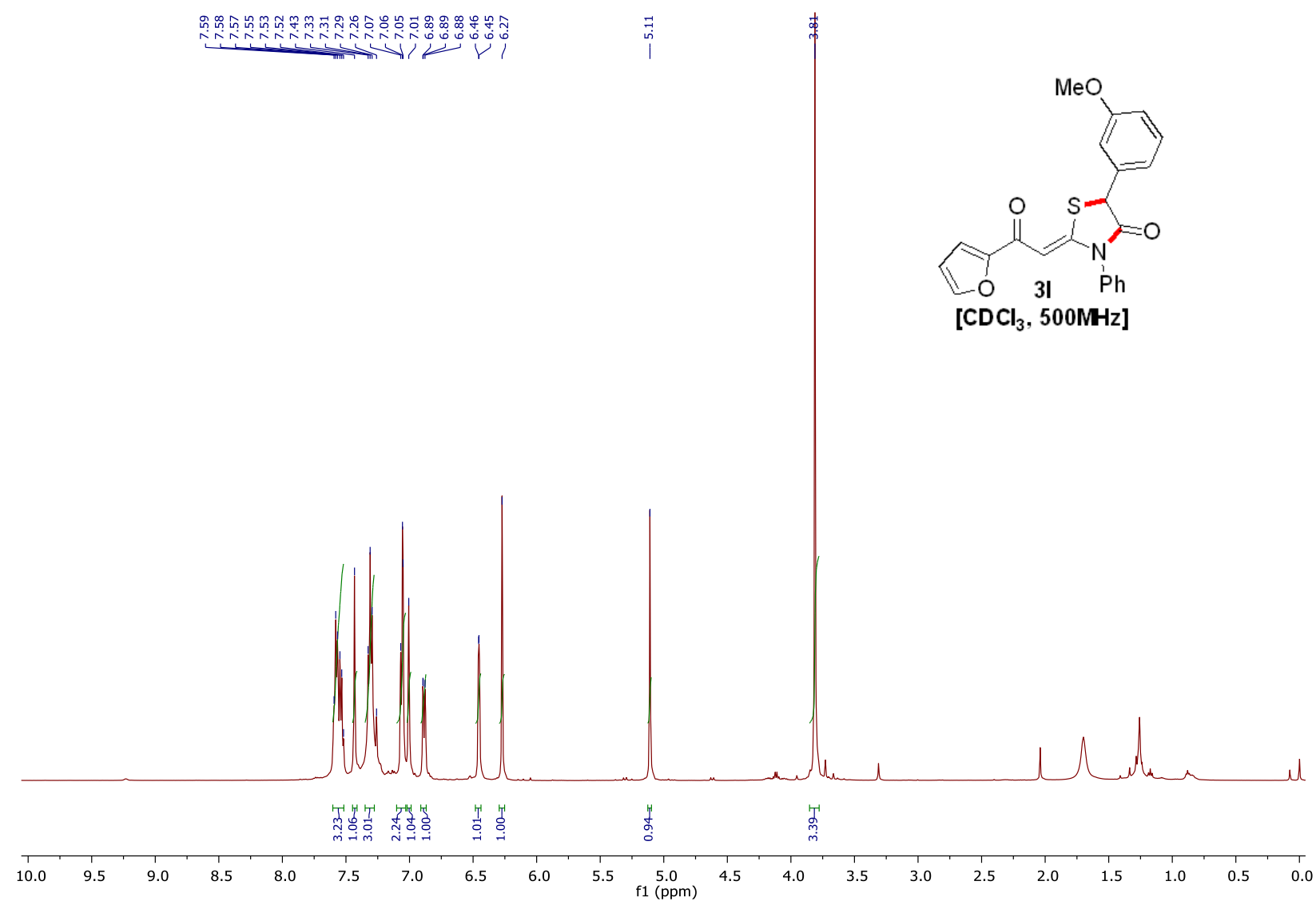

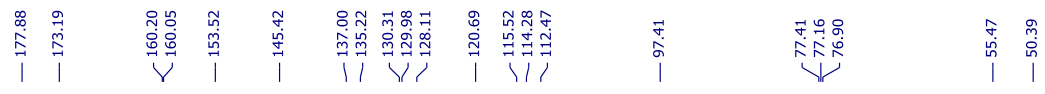

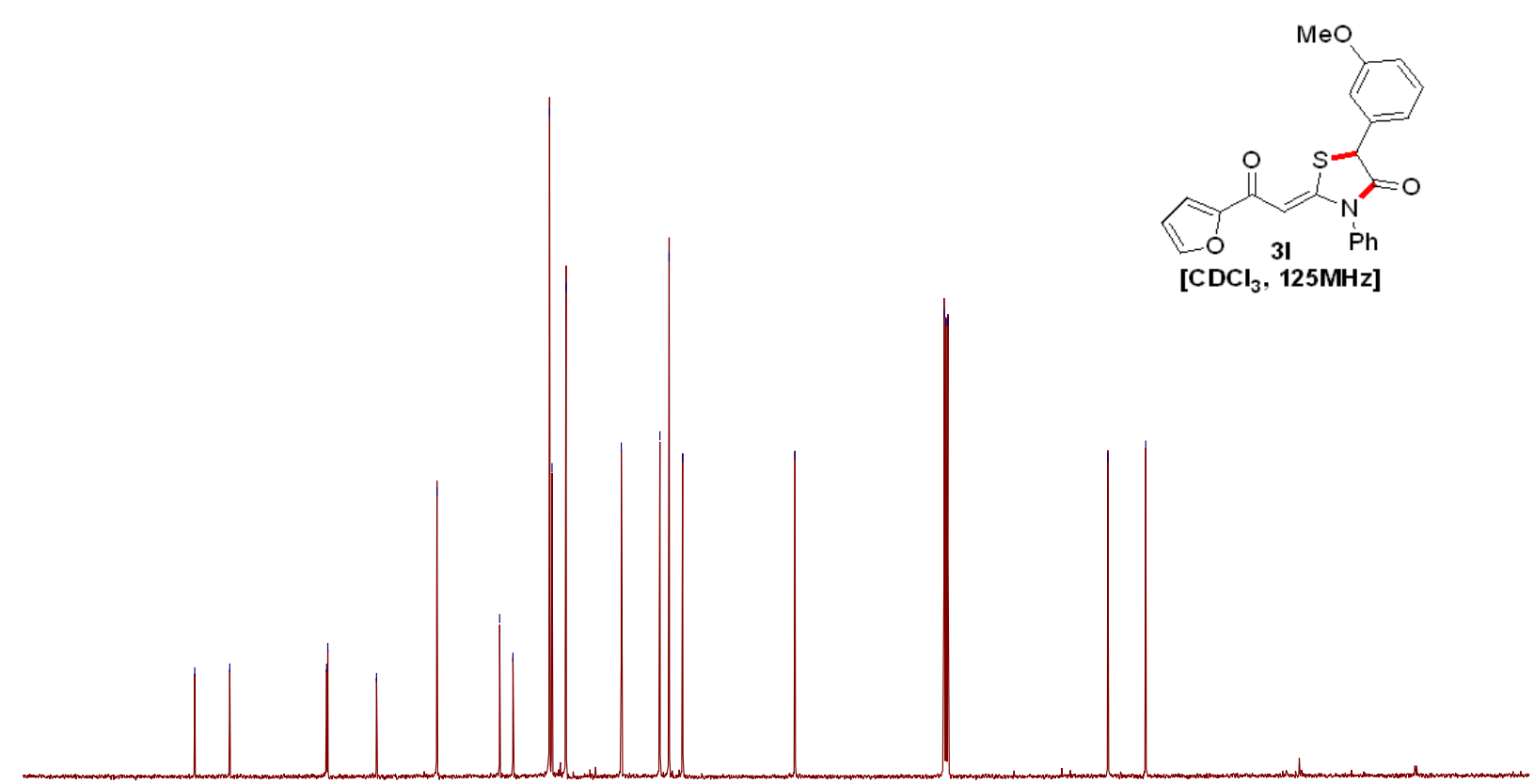

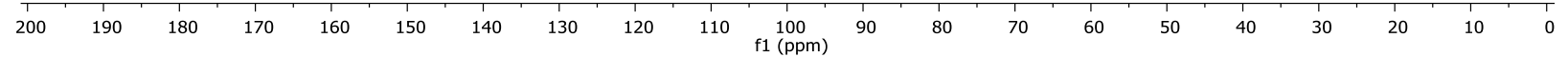


${ }^{1} \mathrm{H}$ and ${ }^{1} \mathrm{H}$ decoupled ${ }^{13} \mathrm{C}$ NMR spectra of compound $\mathbf{3 m}$

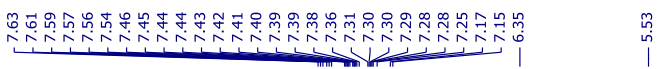

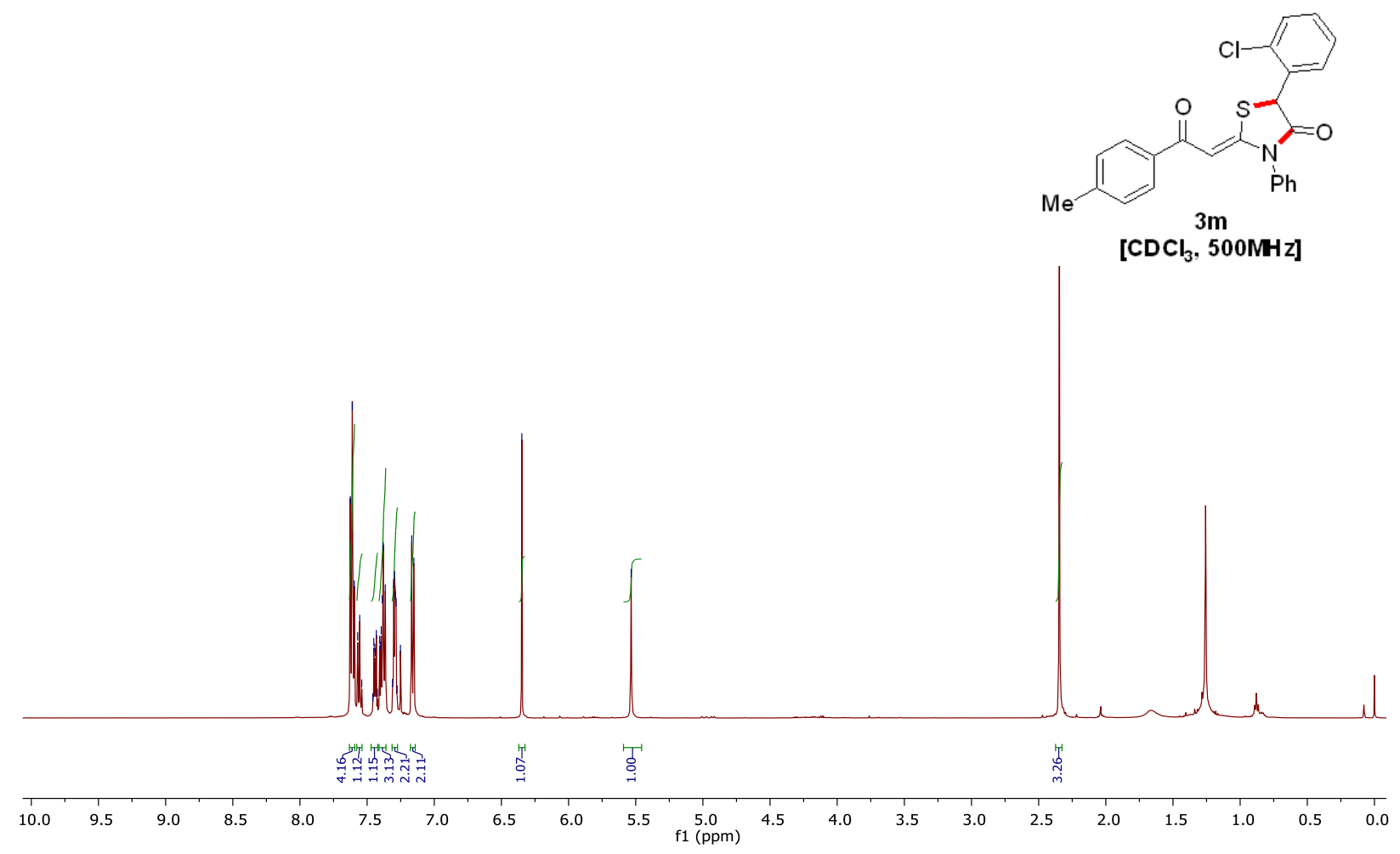

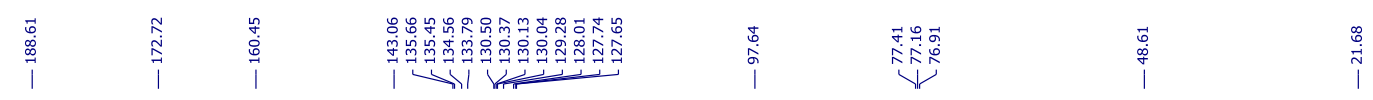

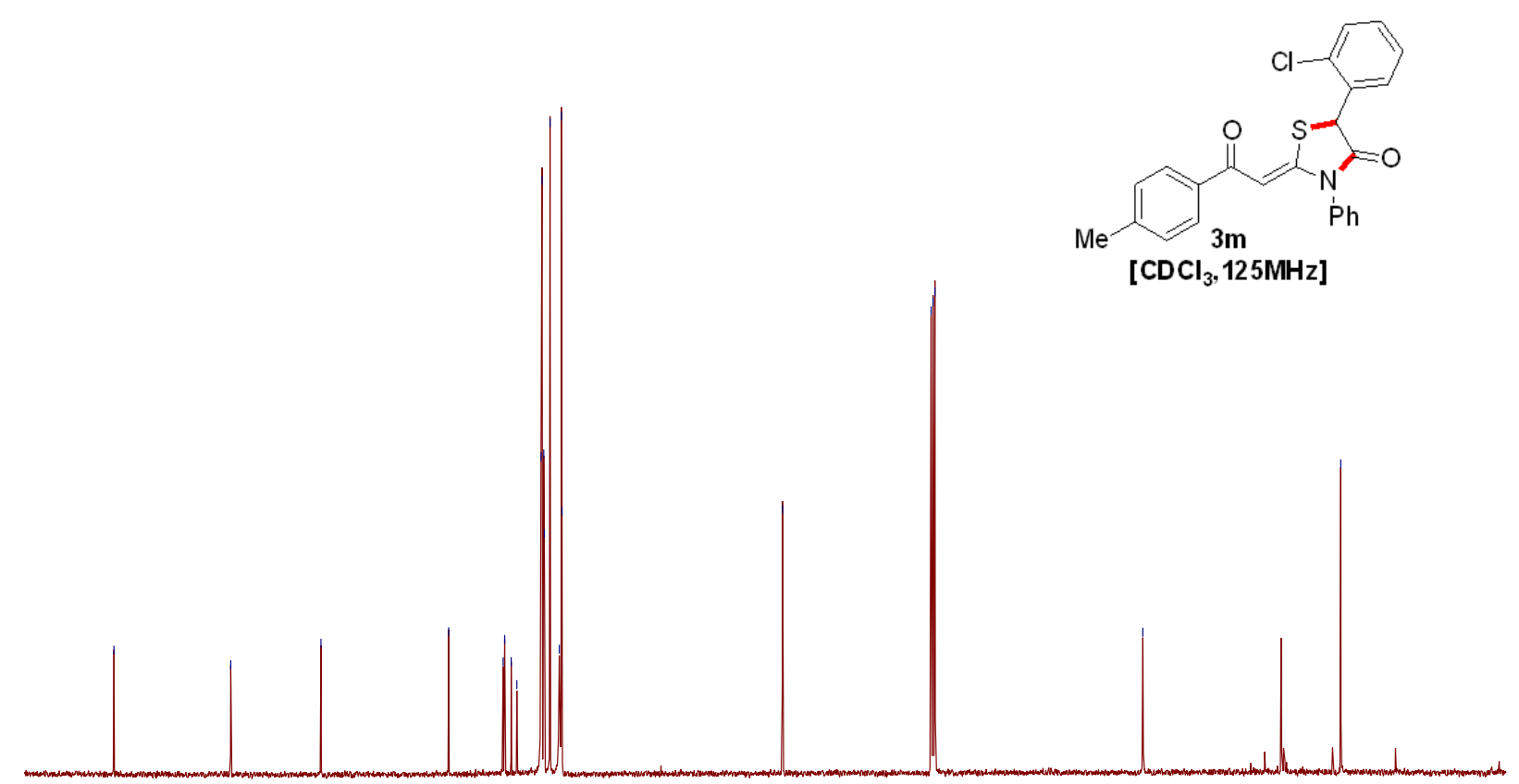

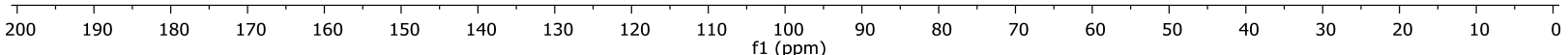


${ }^{1} \mathrm{H}$ and ${ }^{1} \mathrm{H}$ decoupled ${ }^{13} \mathrm{C}$ NMR spectra of compound $\mathbf{3 n}$

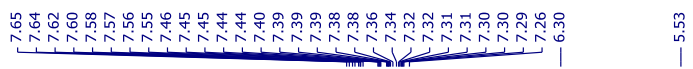
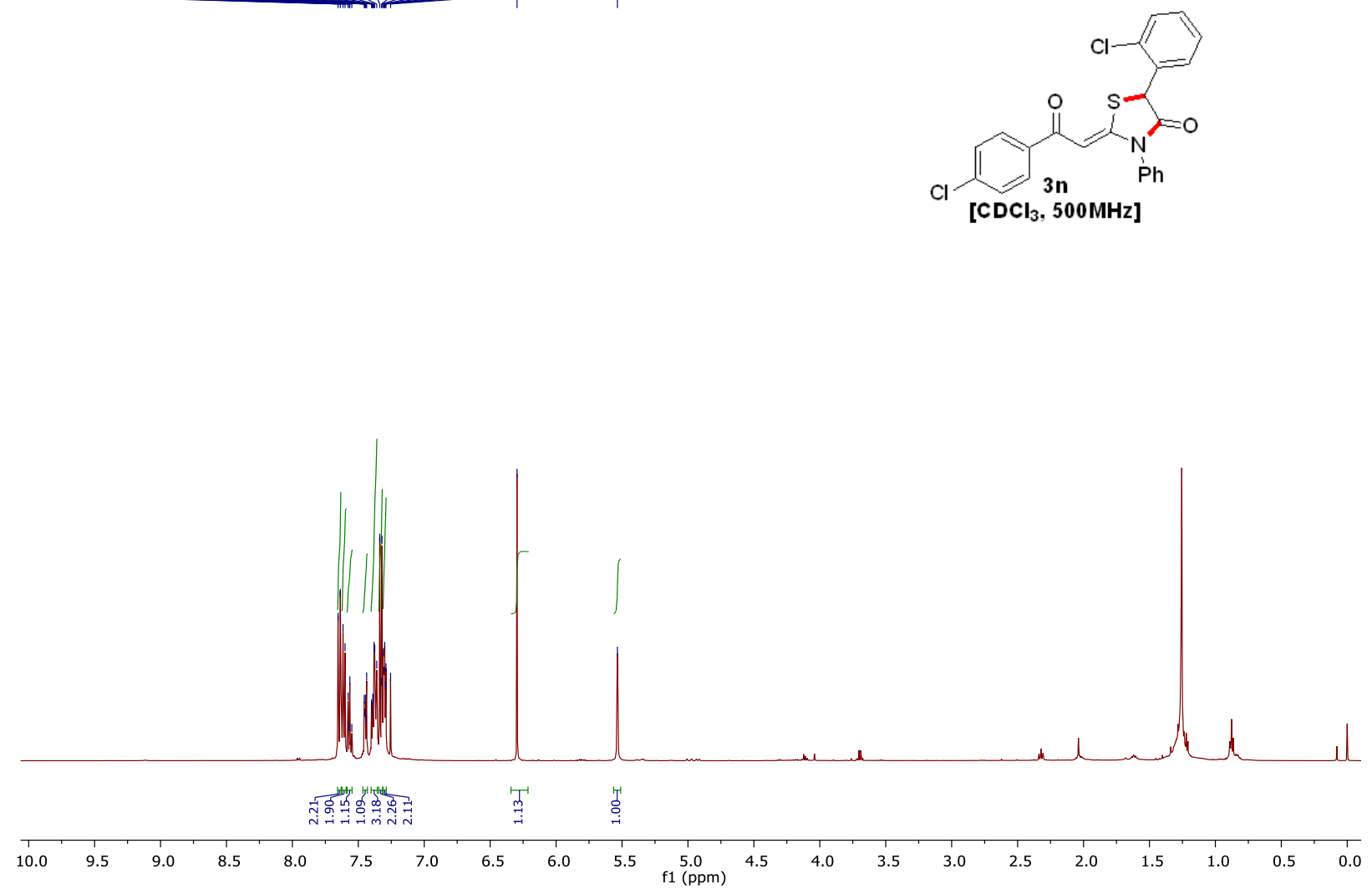

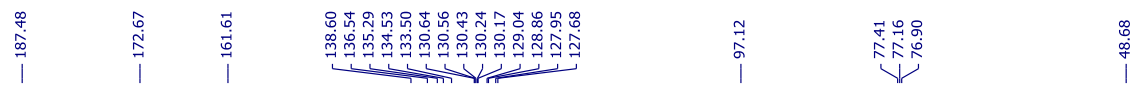

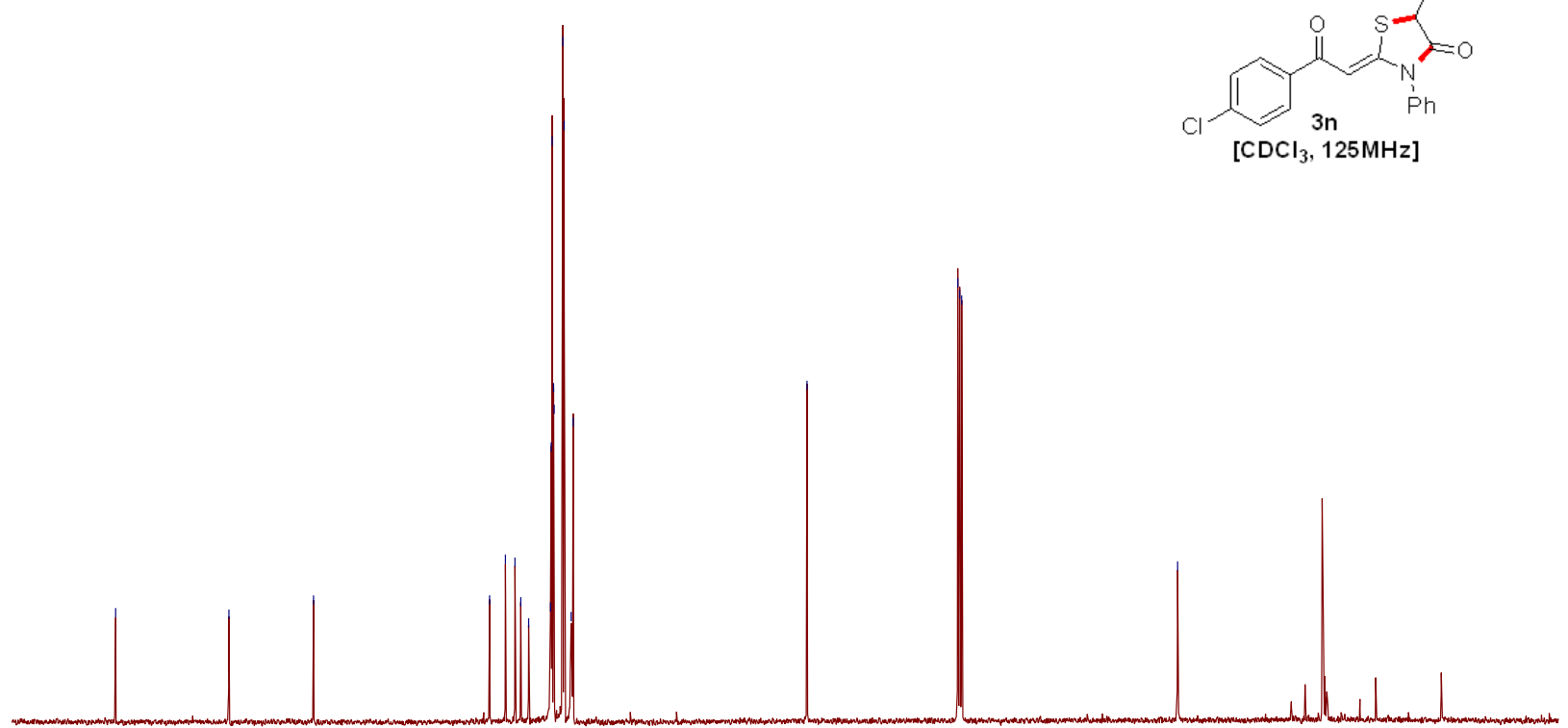

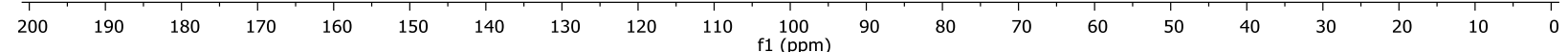


${ }^{1} \mathrm{H}$ and ${ }^{1} \mathrm{H}$ decoupled ${ }^{13} \mathrm{C}$ NMR spectra of compound 30

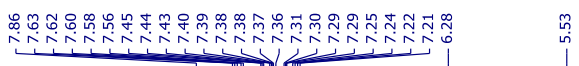

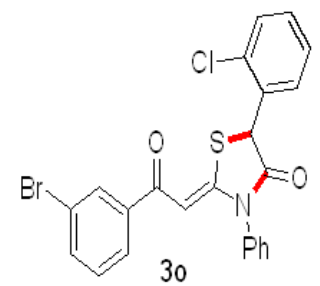

[CDCl, $500 \mathrm{MHz}]$
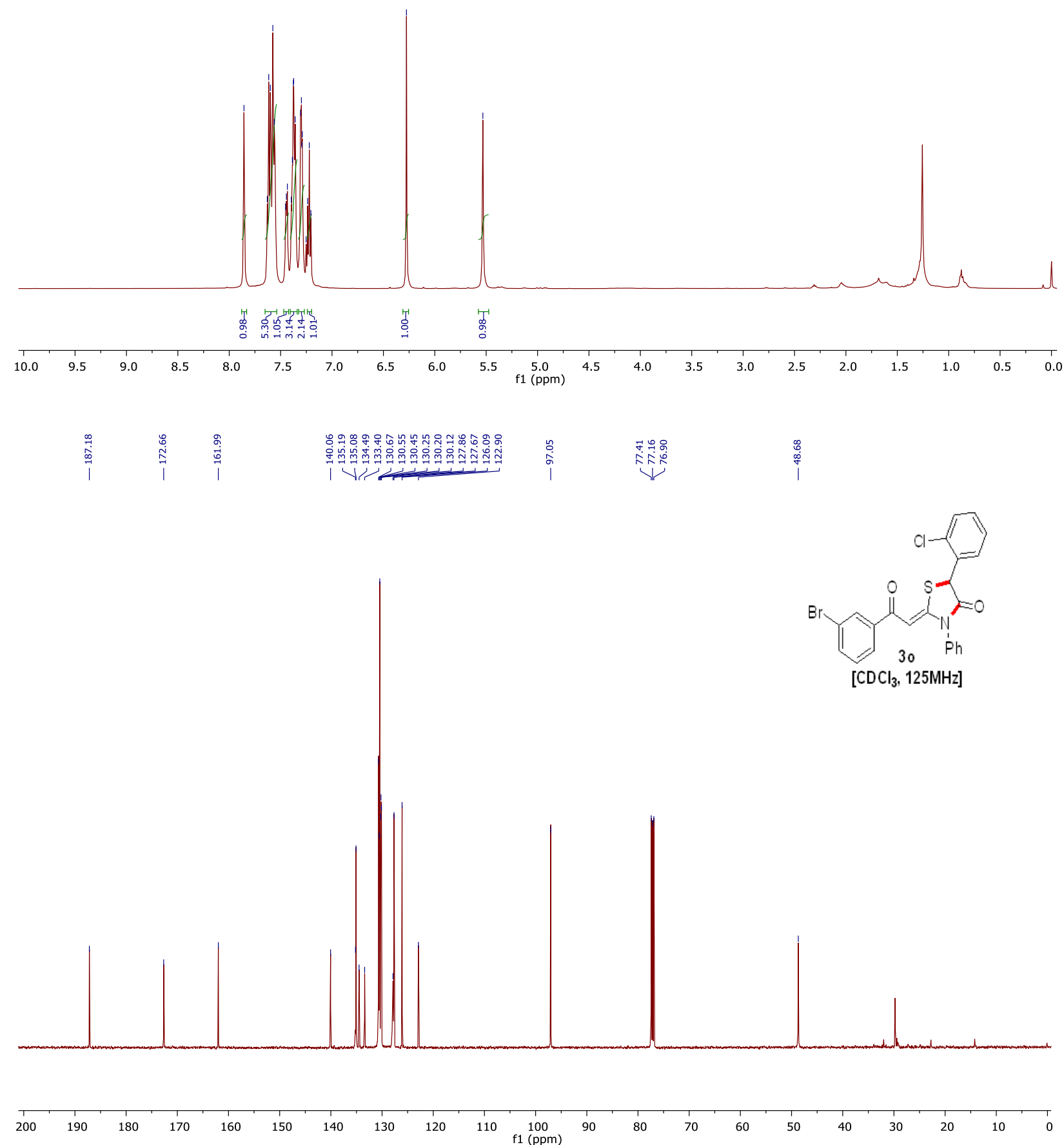
${ }^{1} \mathrm{H}$ and ${ }^{1} \mathrm{H}$ decoupled ${ }^{13} \mathrm{C}$ NMR spectra of compound $\mathbf{3 p}$

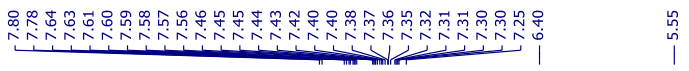

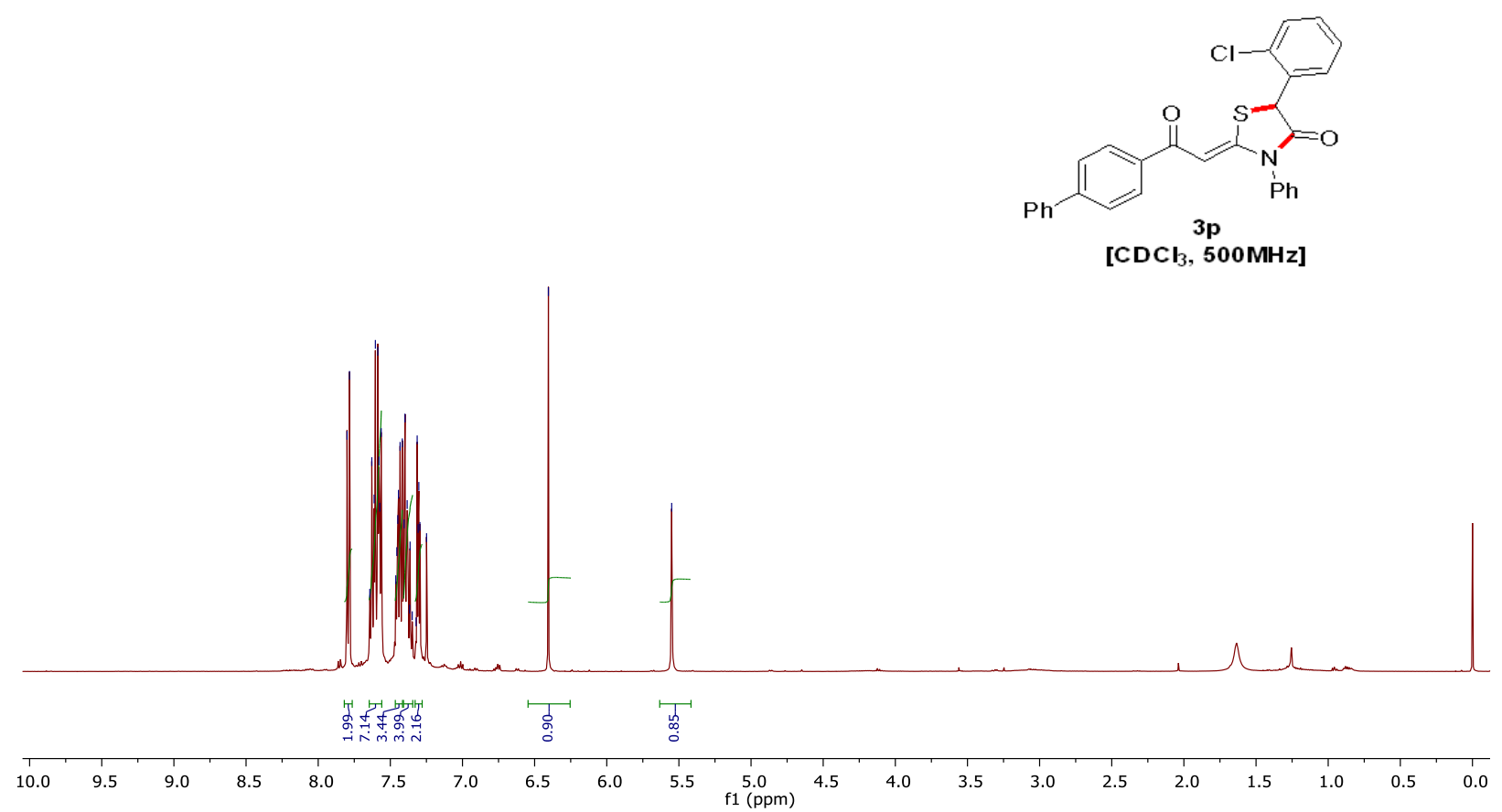

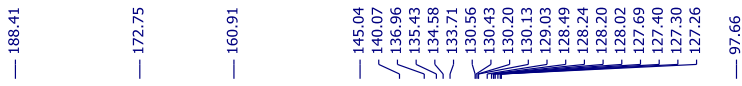

$\underset{\substack{0 \\ \infty}}{\substack{\infty \\ j}}$

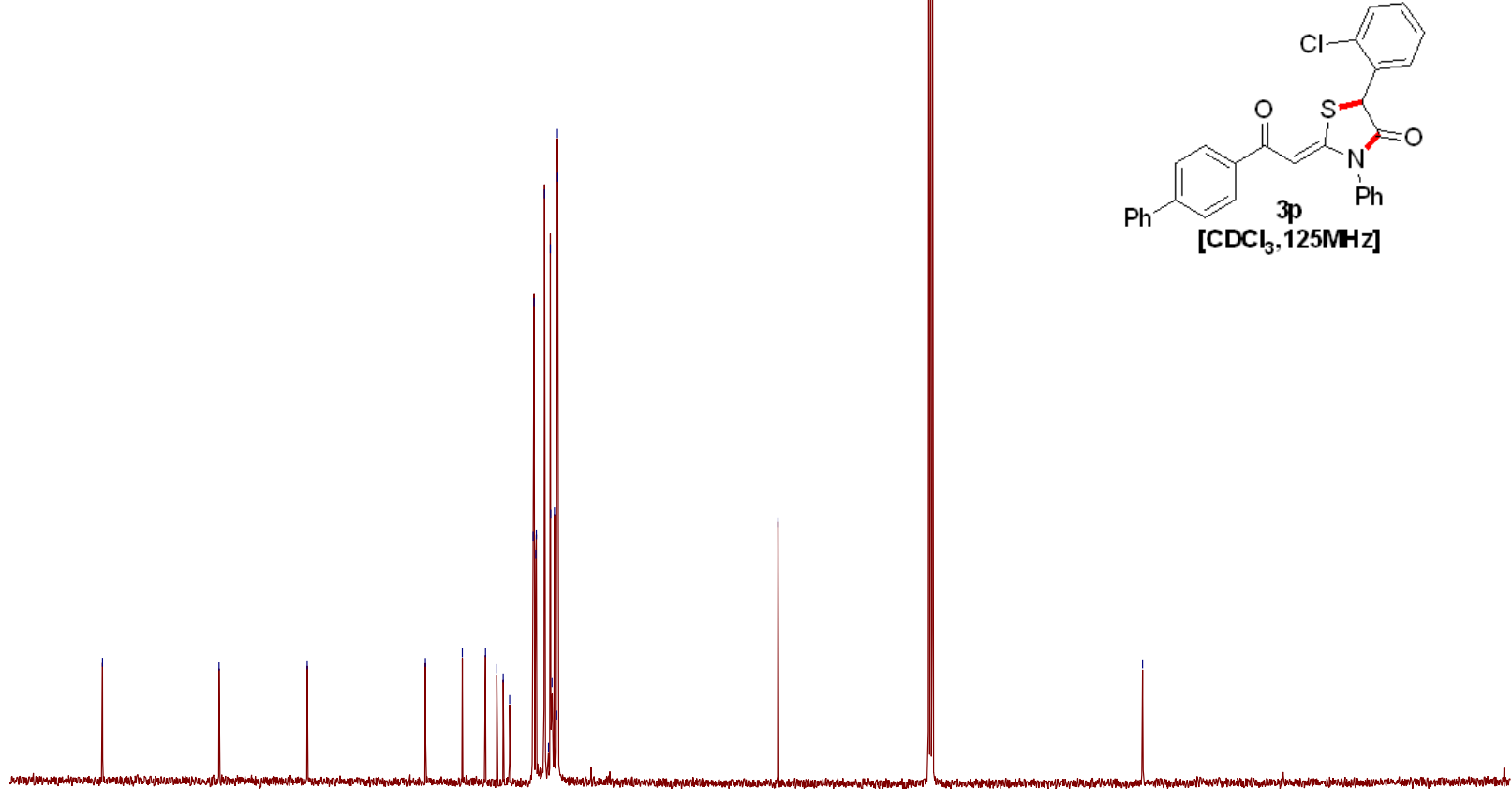

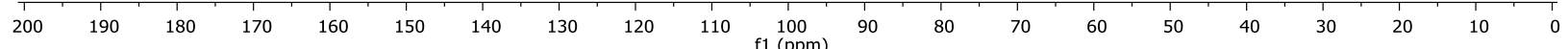


${ }^{1} \mathrm{H}$ and ${ }^{1} \mathrm{H}$ decoupled ${ }^{13} \mathrm{C}$ NMR spectra of compound $\mathbf{3 q}$

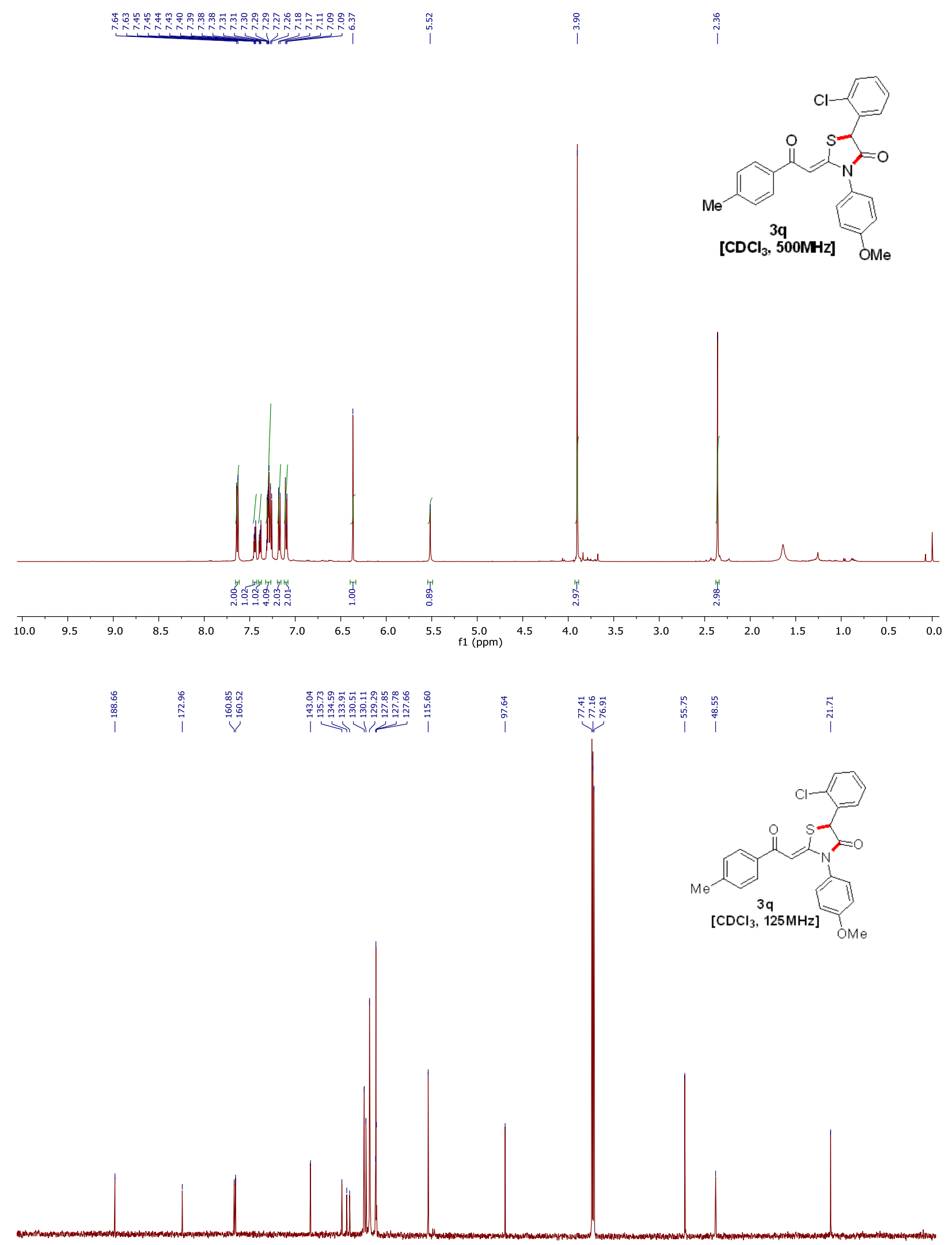

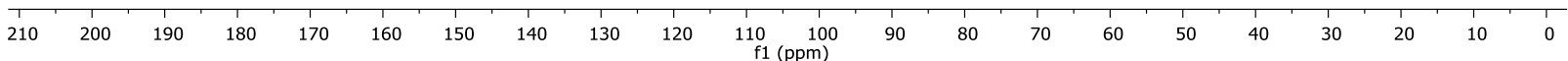


${ }^{1} \mathrm{H}$ and ${ }^{1} \mathrm{H}$ decoupled ${ }^{13} \mathrm{C}$ NMR spectra of compound $\mathbf{3 r}$

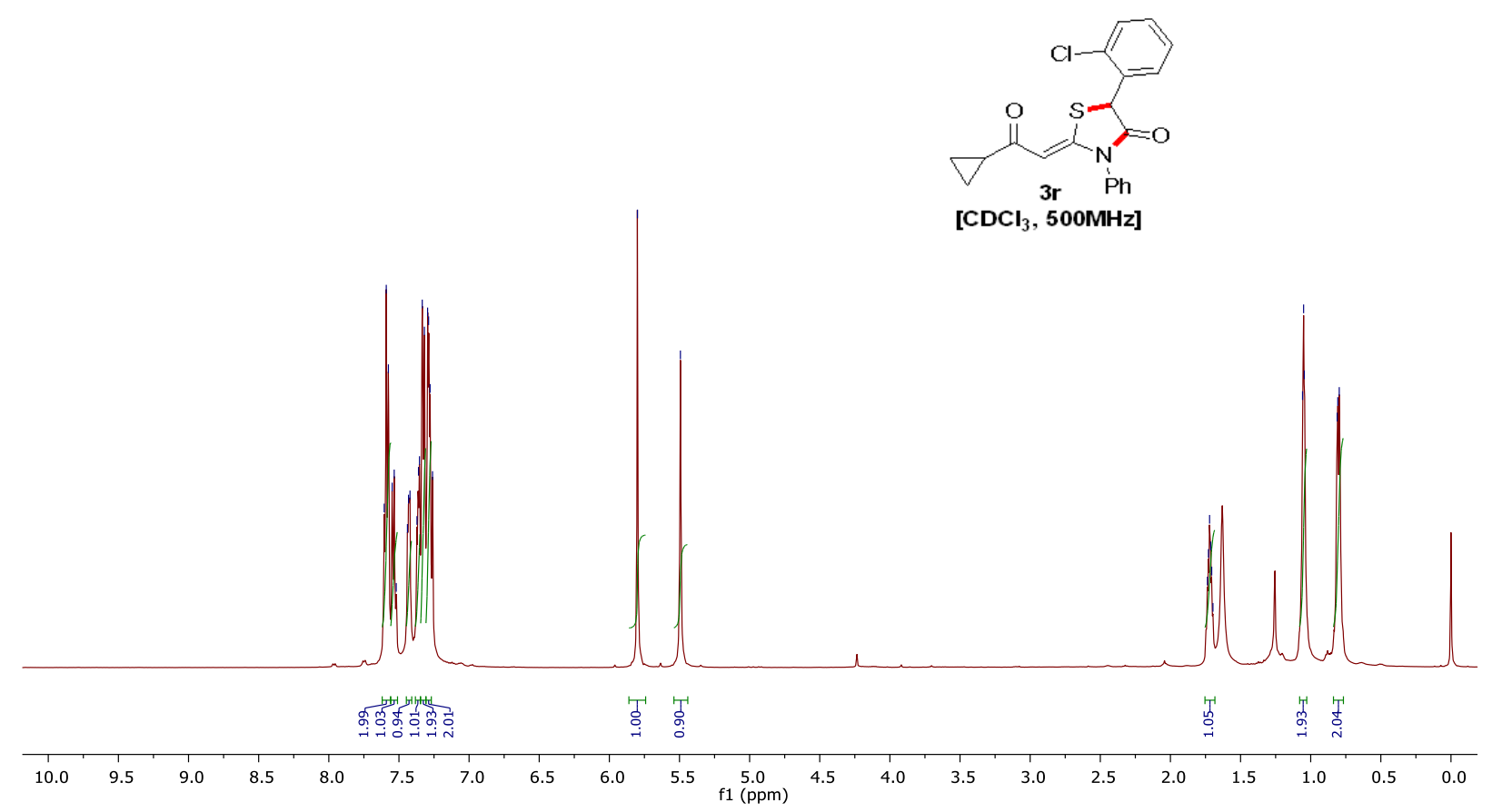

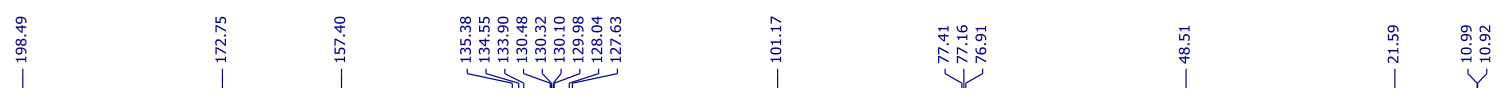

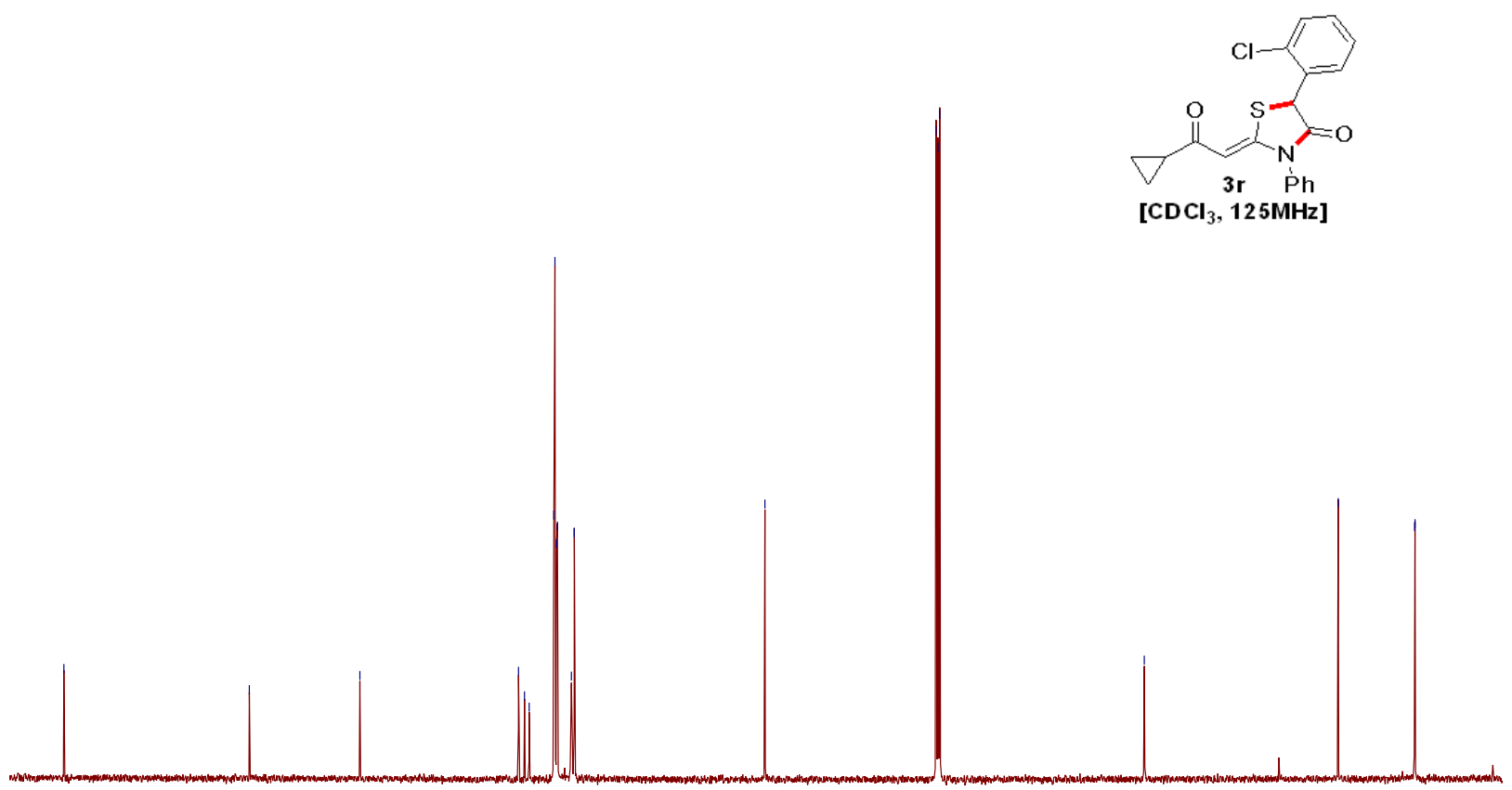

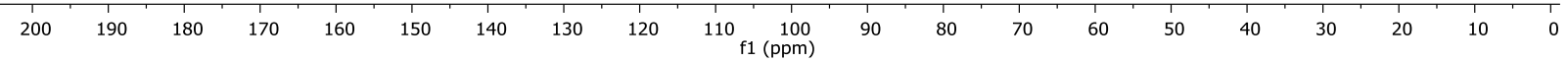


${ }^{1} \mathrm{H}$ and ${ }^{1} \mathrm{H}$ decoupled ${ }^{13} \mathrm{C}$ NMR spectra of compound $3 \mathrm{~s}$
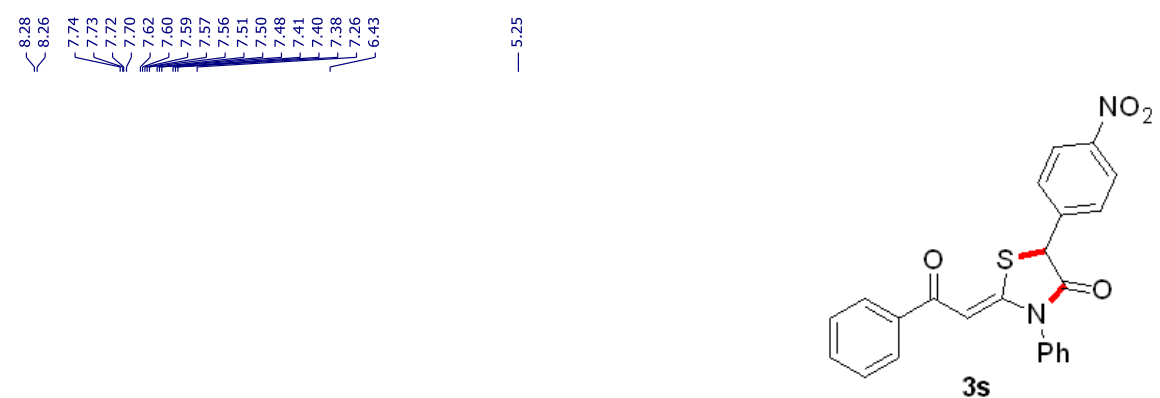

$\left[\mathrm{CDCl}_{3}, 500 \mathrm{MHz}\right]$

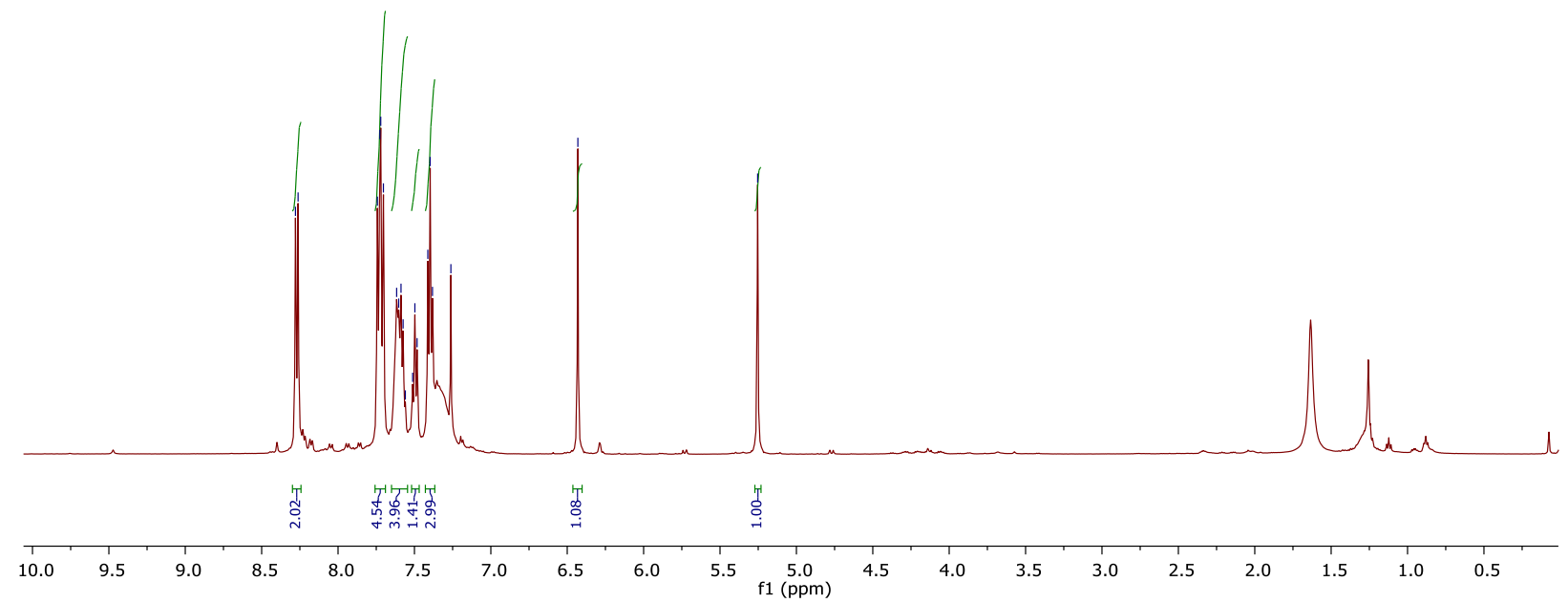

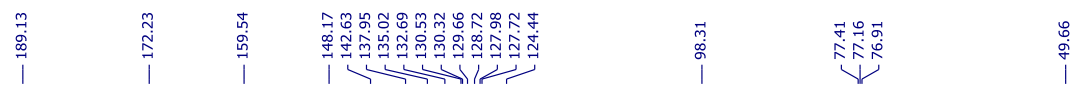

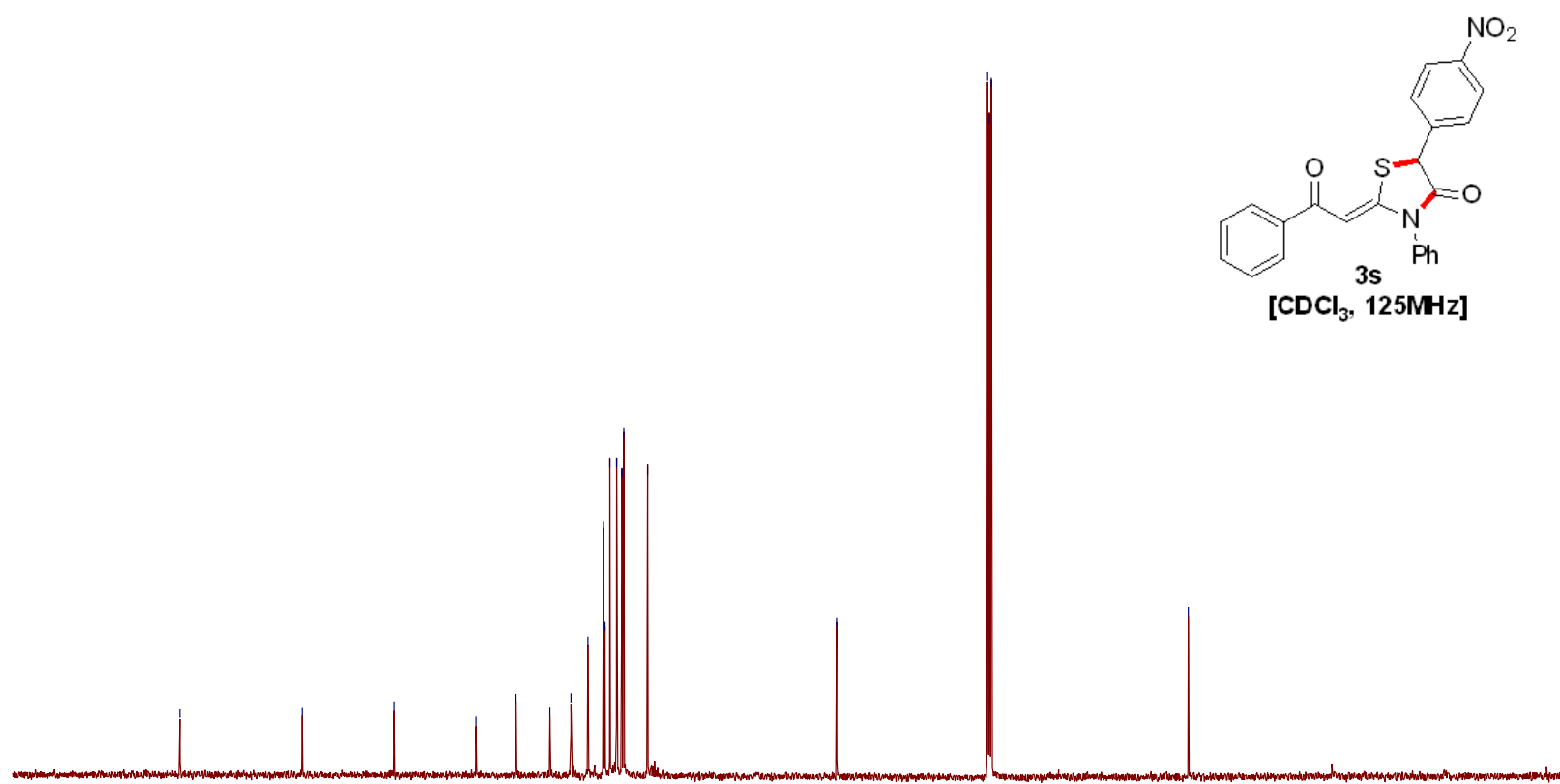

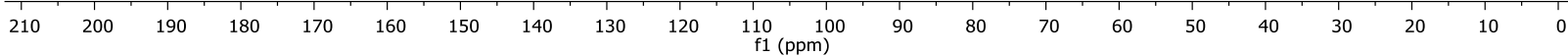


${ }^{1} \mathrm{H}$ and ${ }^{1} \mathrm{H}$ decoupled ${ }^{13} \mathrm{C}$ NMR spectra of compound $\mathbf{3 t}$
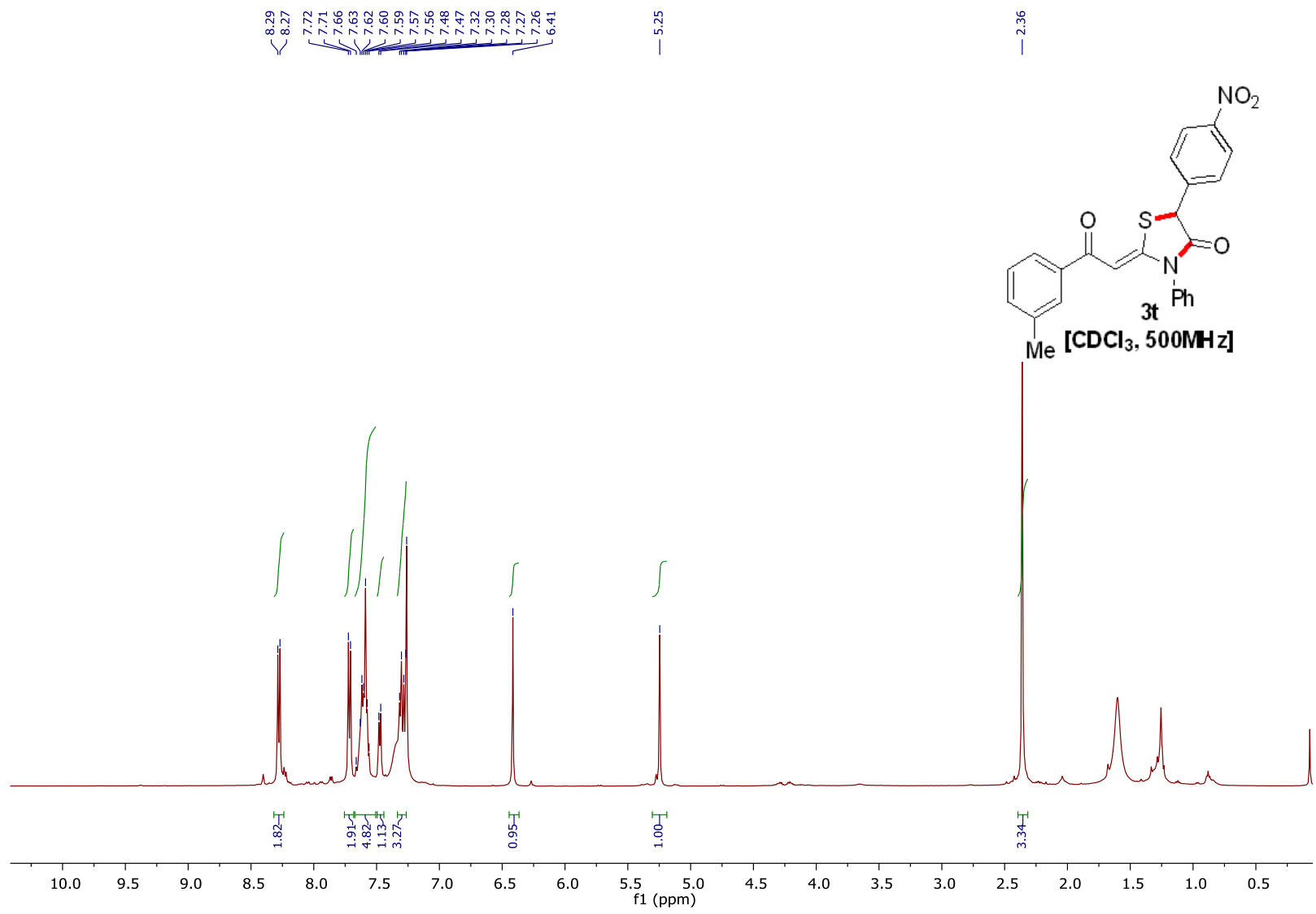

\section{|}

$\begin{array}{rllllllllll}210 & 200 & 190 & 180 & 170 & 160 & 150 & 140 & 130 & 120 & \begin{array}{l}110 \\ \mathrm{f} 1(\mathrm{ppm})\end{array}\end{array}$ 
${ }^{1} \mathrm{H}$ and ${ }^{1} \mathrm{H}$ decoupled ${ }^{13} \mathrm{C}$ NMR spectra of compound $3 \mathbf{u}$
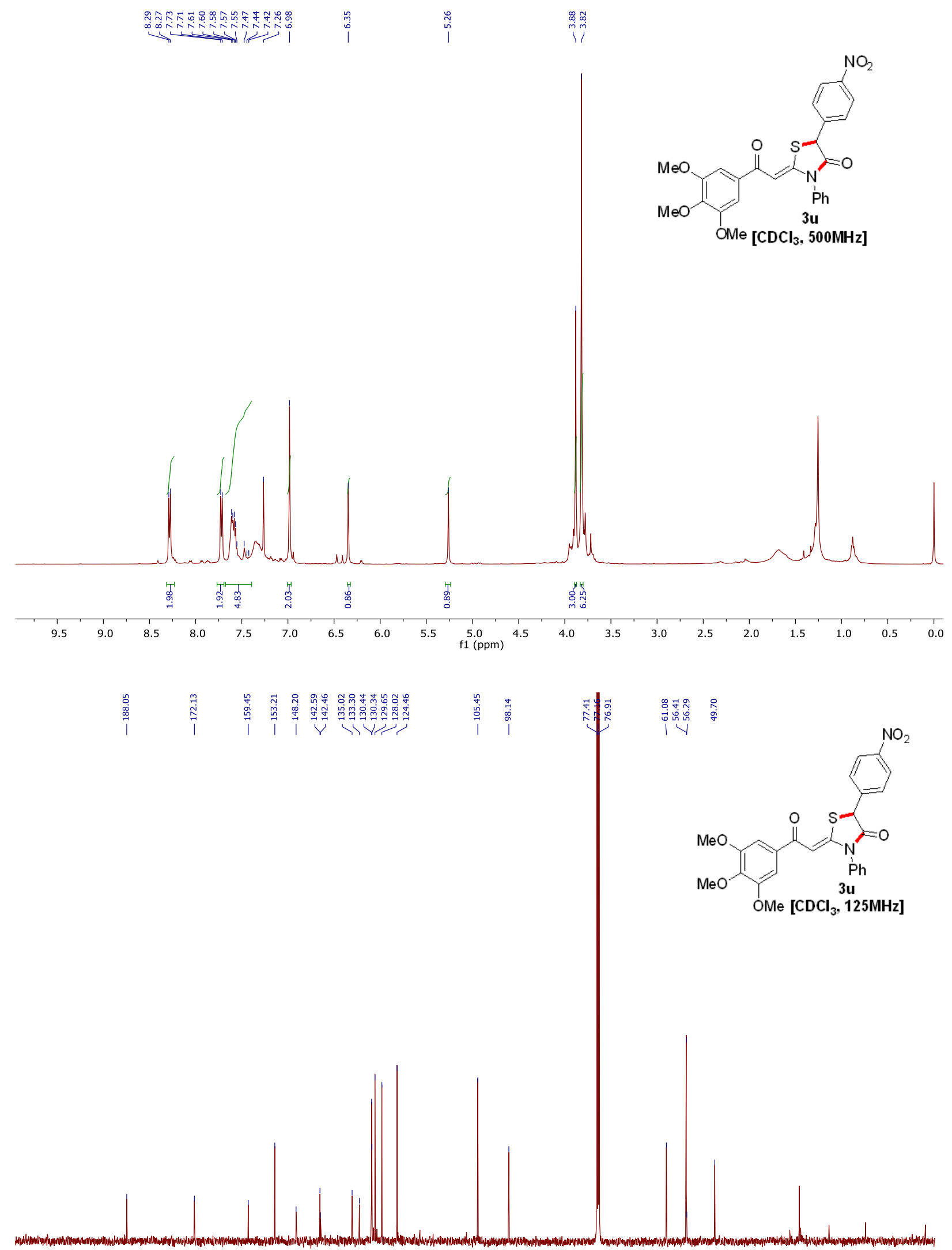

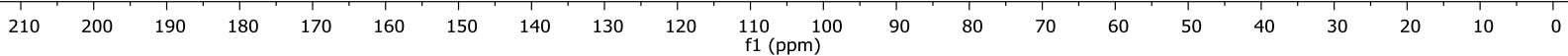


${ }^{1} \mathrm{H}$ and ${ }^{1} \mathrm{H}$ decoupled ${ }^{13} \mathrm{C}$ NMR spectra of compound $\mathbf{3 v}$

1.
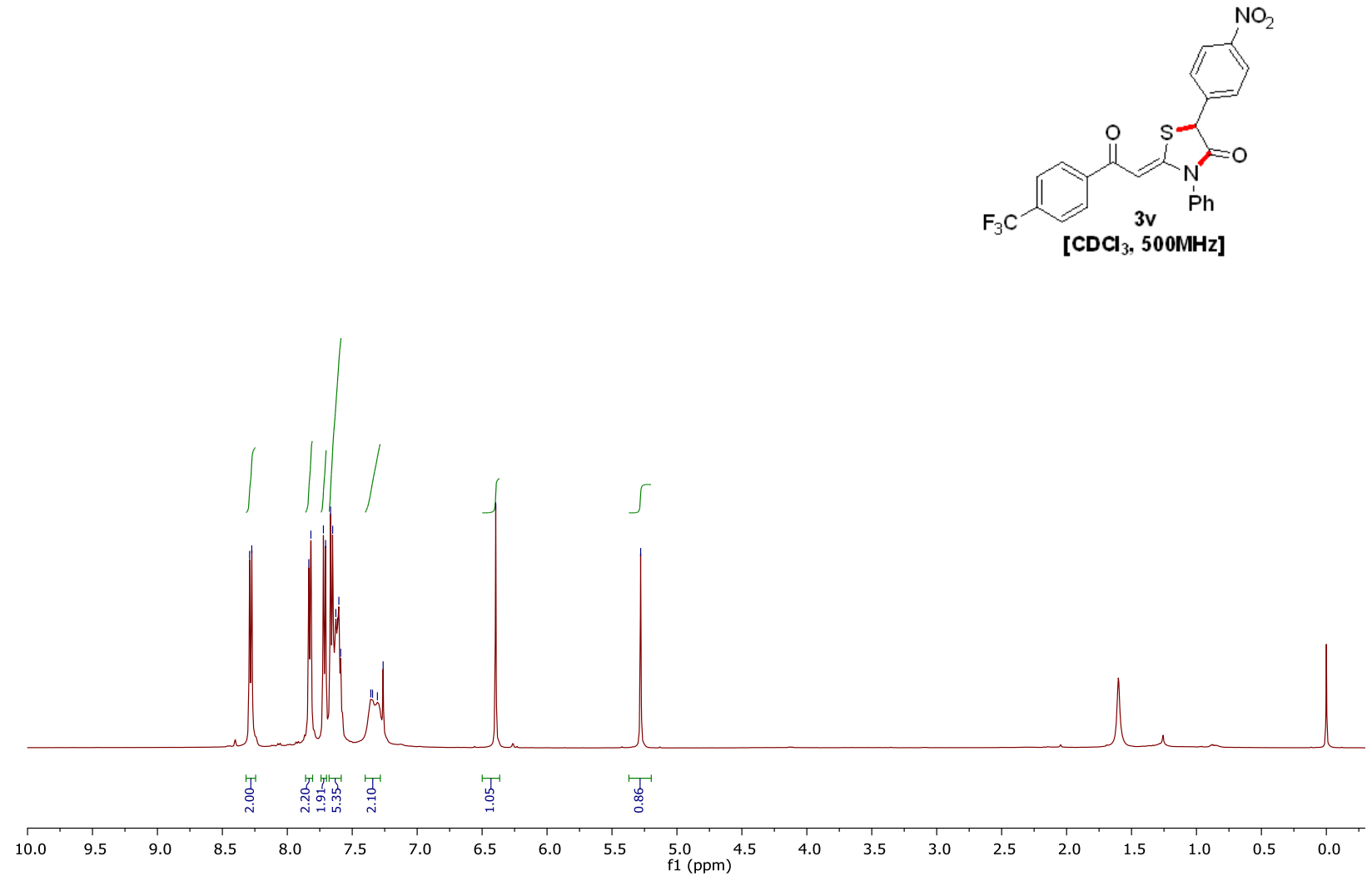

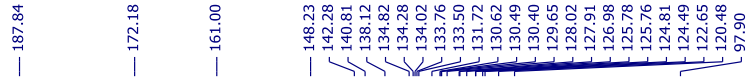
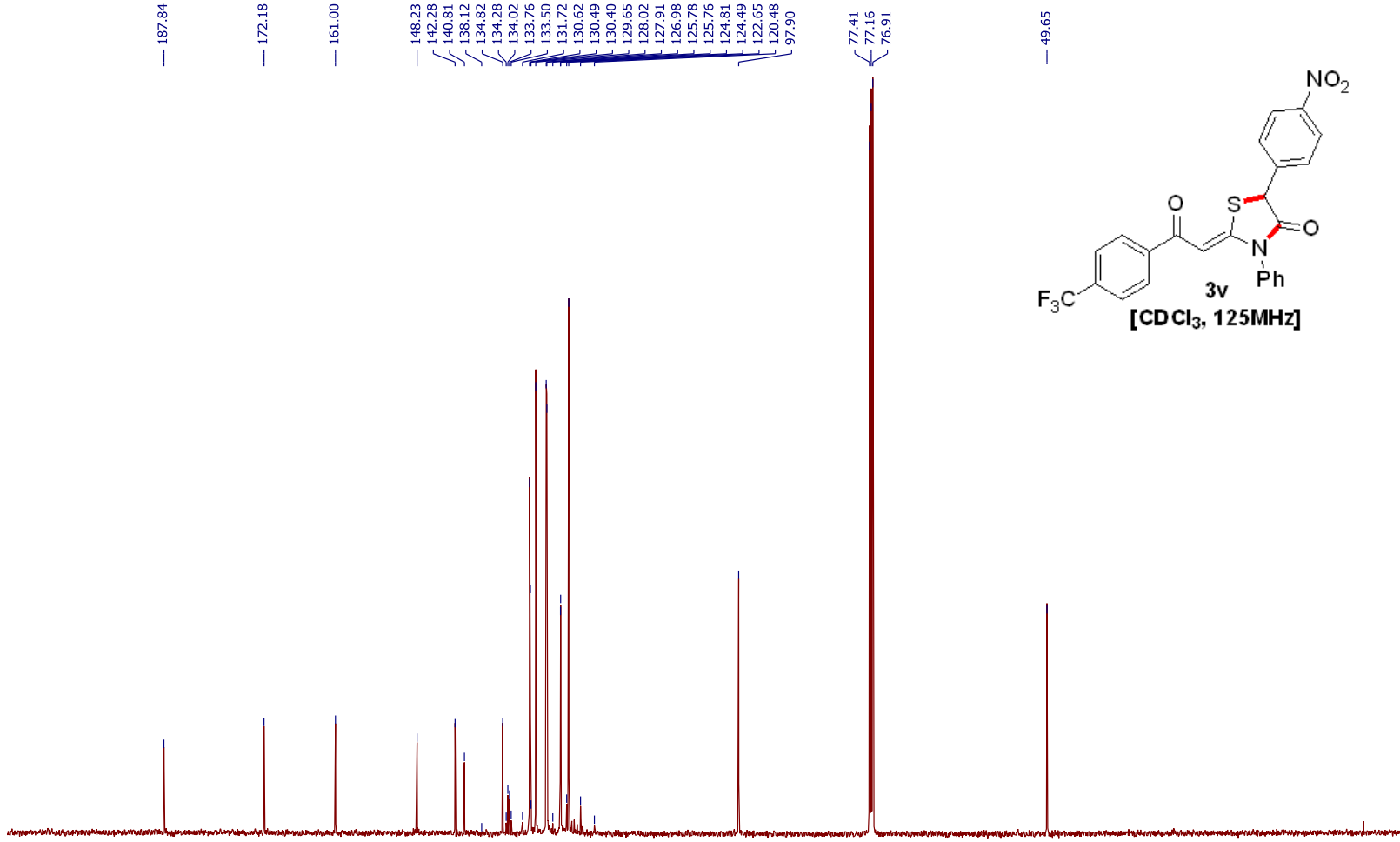

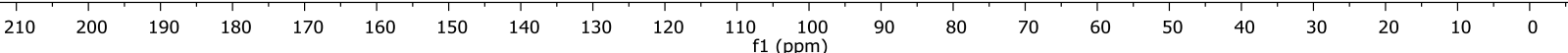


${ }^{1} \mathrm{H}$ and ${ }^{1} \mathrm{H}$ decoupled ${ }^{13} \mathrm{C}$ NMR spectra of compound $\mathbf{3 w}$

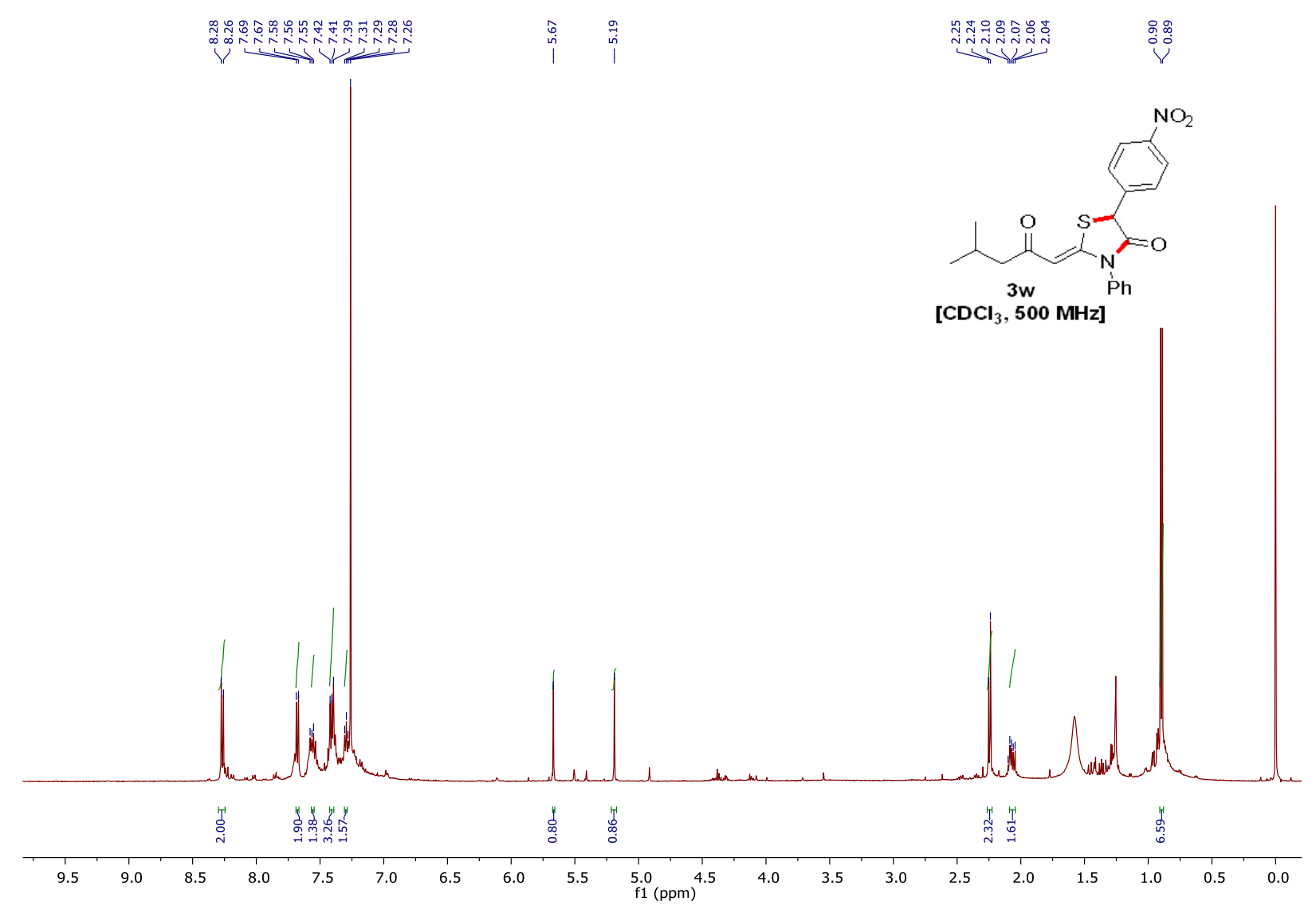

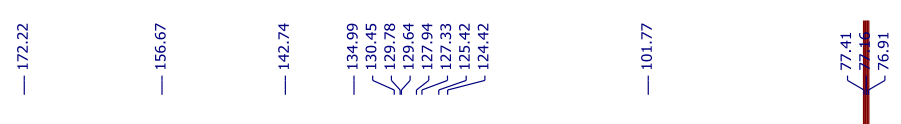

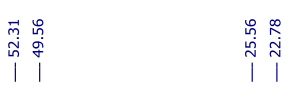

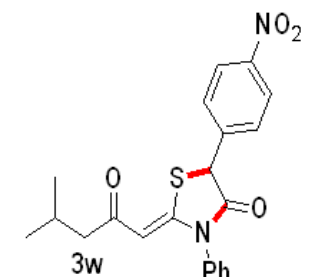

[CDCl, $125 \mathrm{MHz}]$

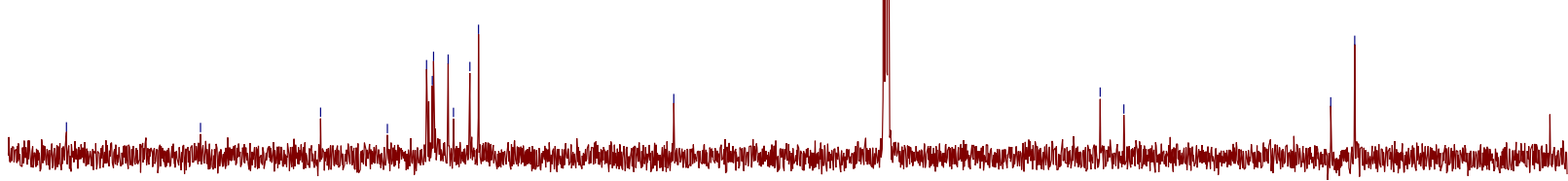

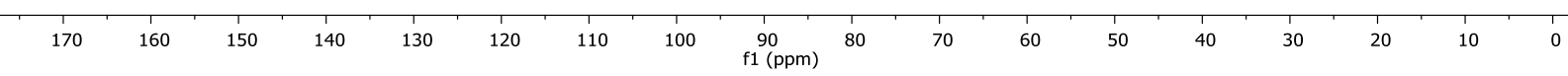


${ }^{1} \mathrm{H}$ and ${ }^{1} \mathrm{H}$ decoupled ${ }^{13} \mathrm{C}$ NMR spectra of compound $\mathbf{3 x}$
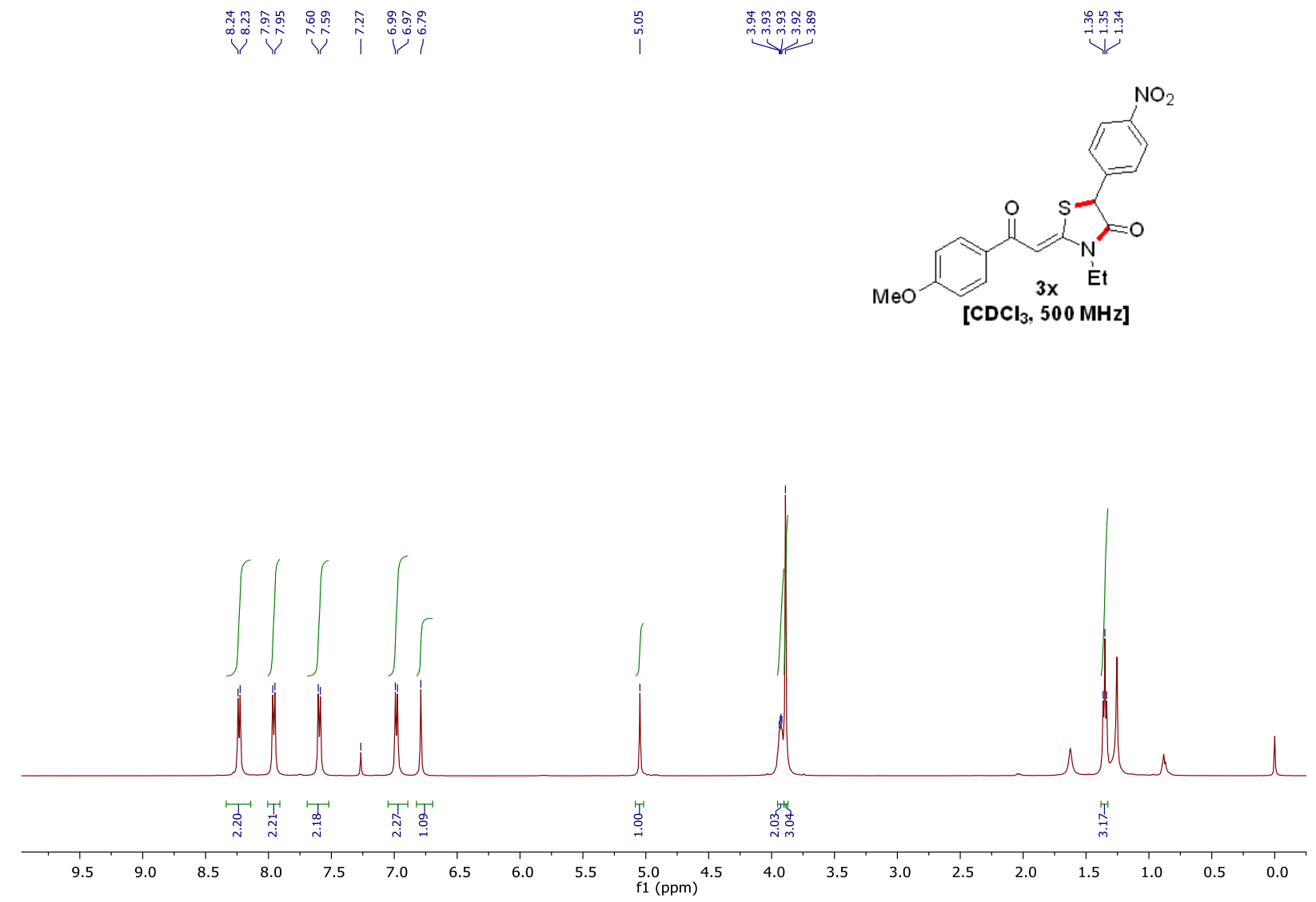

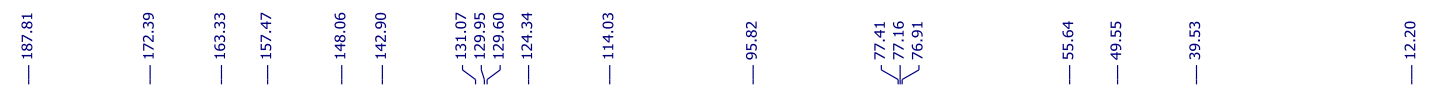
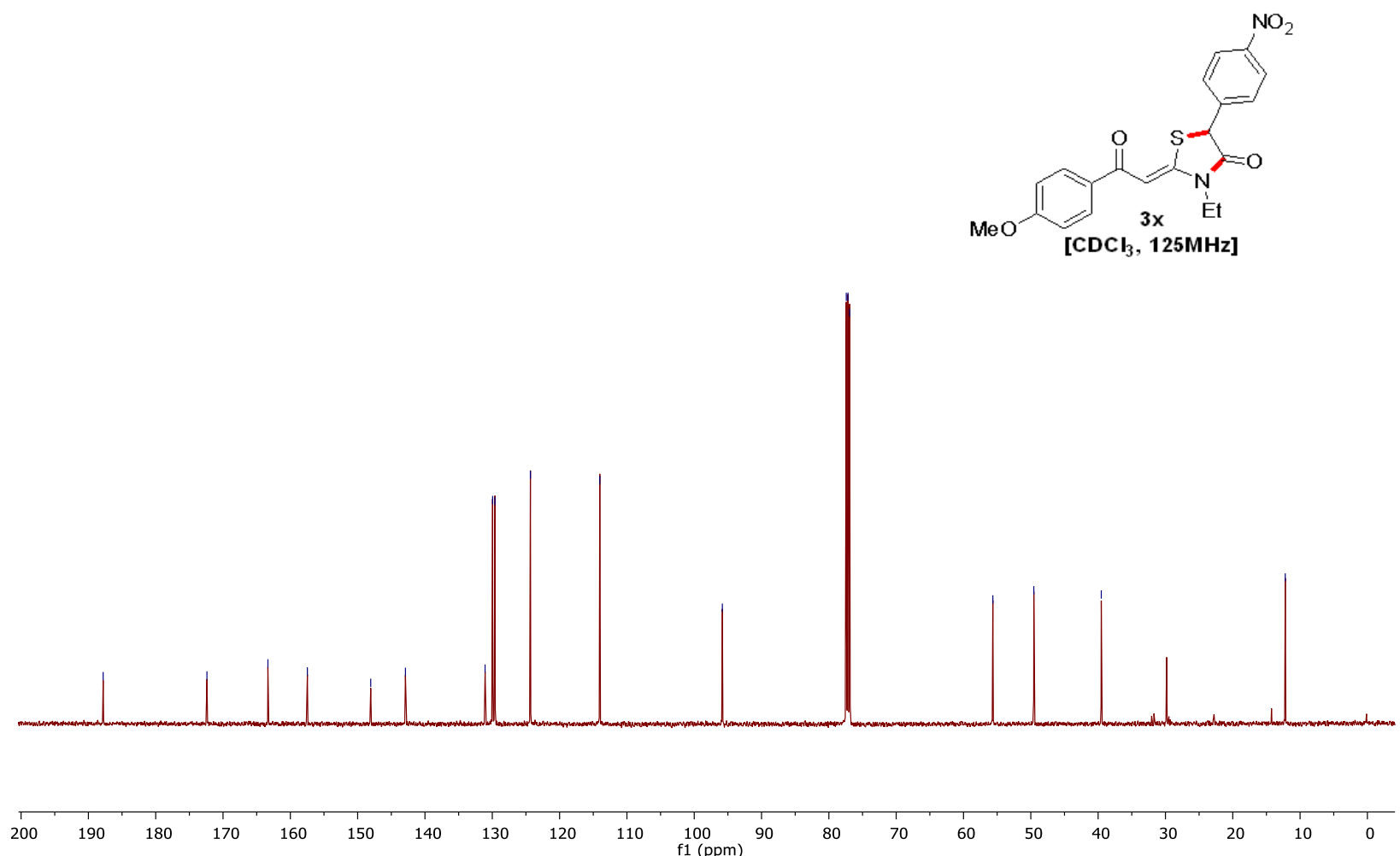
${ }^{1} \mathrm{H}$ and ${ }^{1} \mathrm{H}$ decoupled ${ }^{13} \mathrm{C}$ NMR spectra of compound $\mathbf{3 y}$

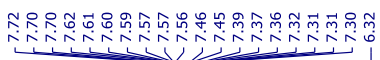

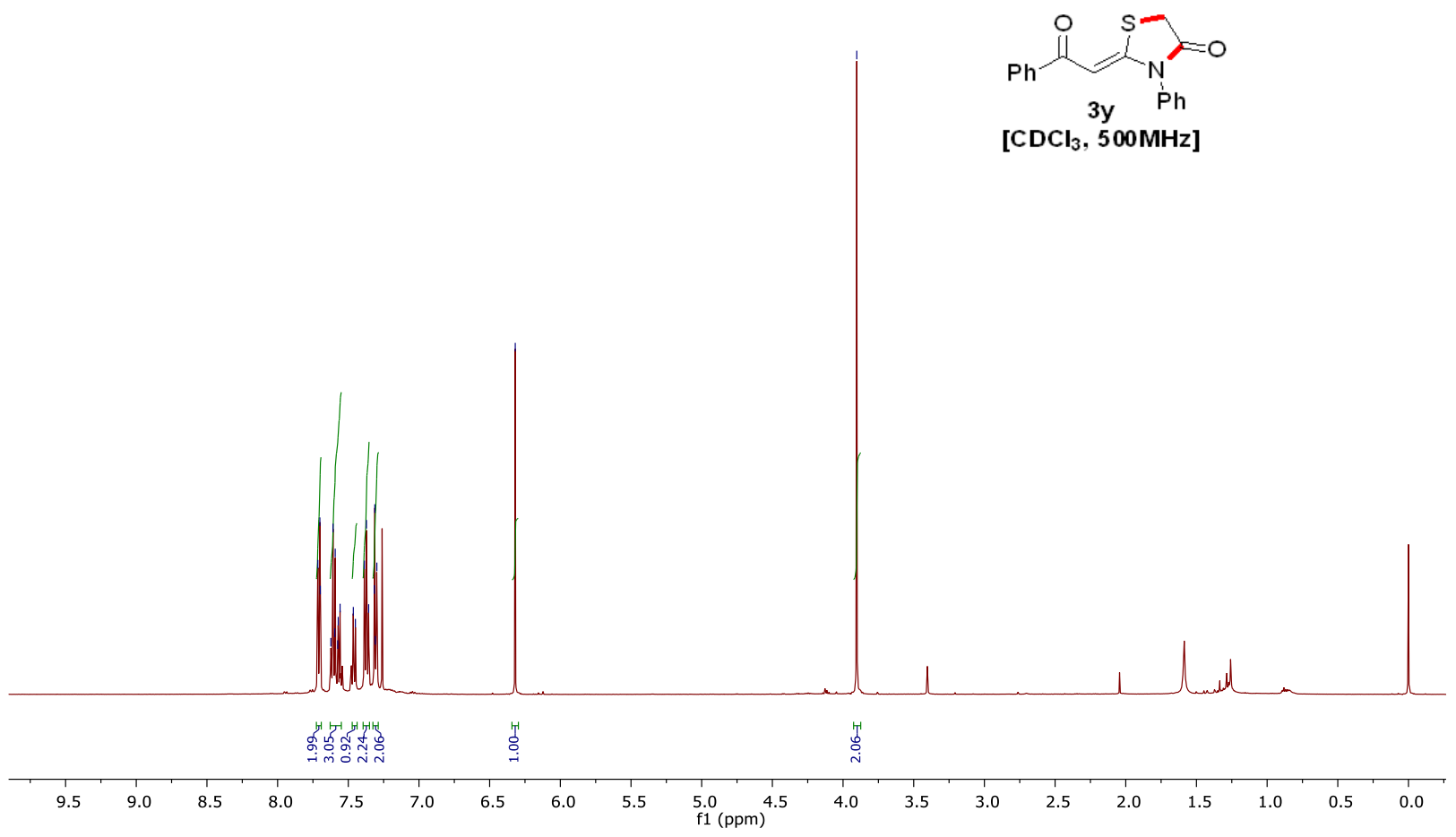

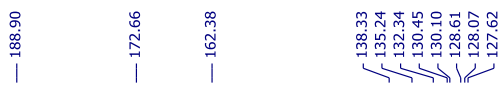

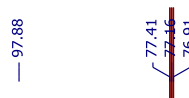

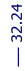

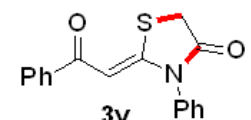

3y $\mathrm{Ph}$

$\left[\mathrm{CDCl}_{3}, 125 \mathrm{MHz}\right]$

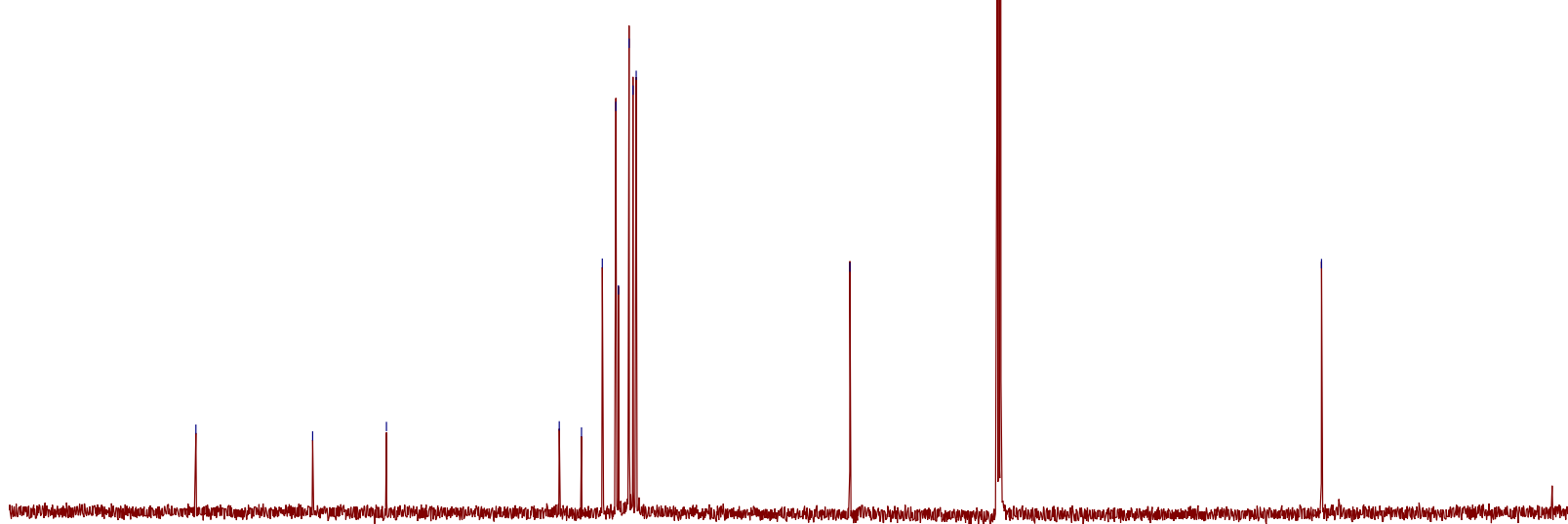

$\begin{array}{llllllllllllllllllllllllllll} & 210 & 200 & 190 & 180 & 170 & 160 & 150 & 140 & 130 & 120 & 110 & 100 & 90 & 80 & 70 & 60 & 50 & 40 & 30 & 20 & 10 & 0\end{array}$ 
${ }^{1} \mathrm{H}$ and ${ }^{1} \mathrm{H}$ decoupled ${ }^{13} \mathrm{C}$ NMR spectra of compound $\mathbf{3 z}$

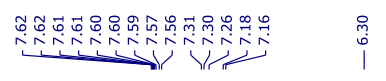
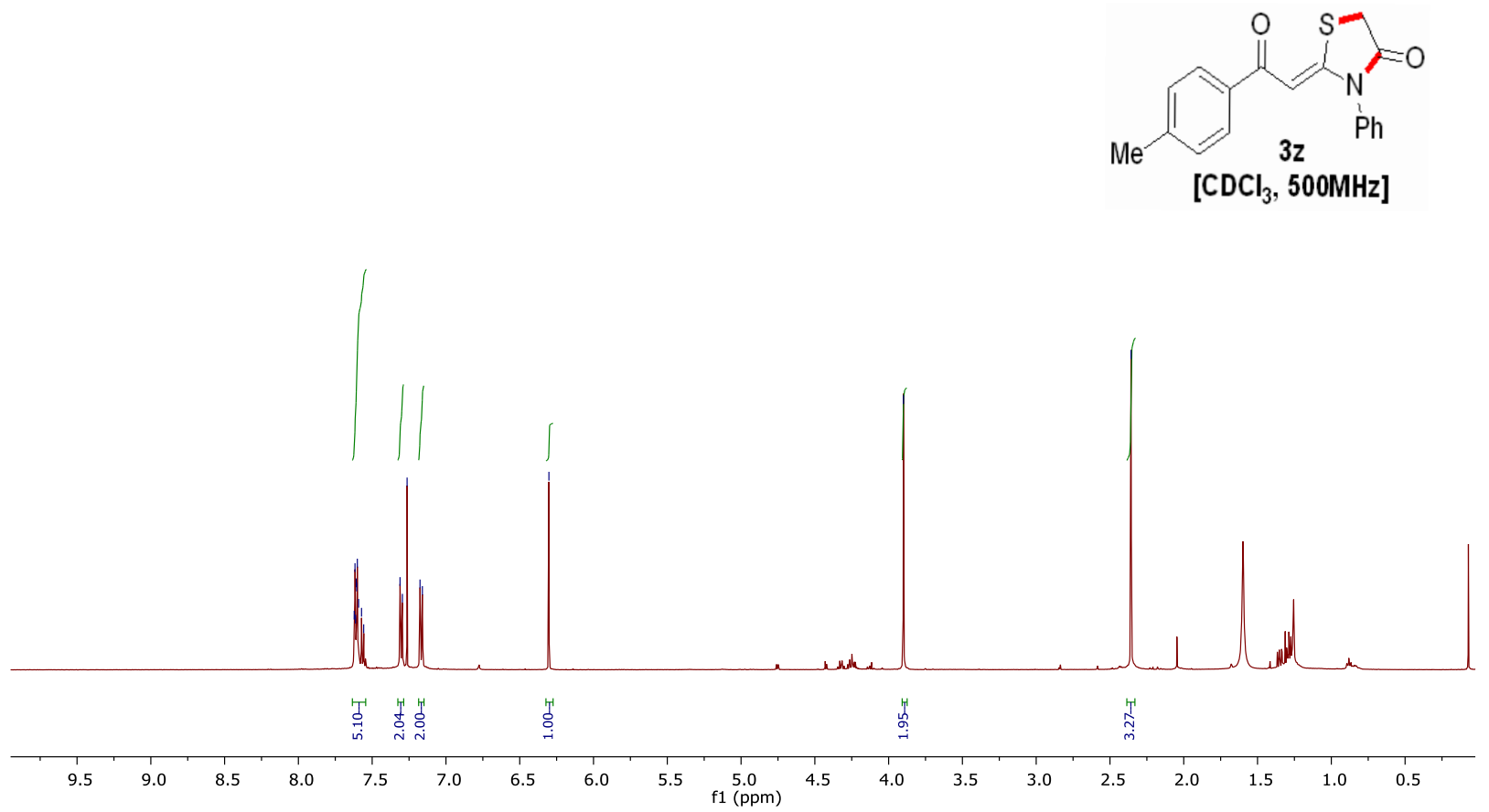

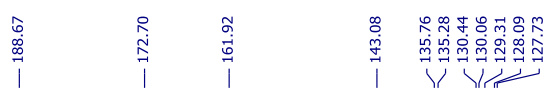

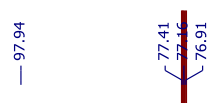

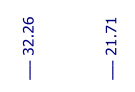
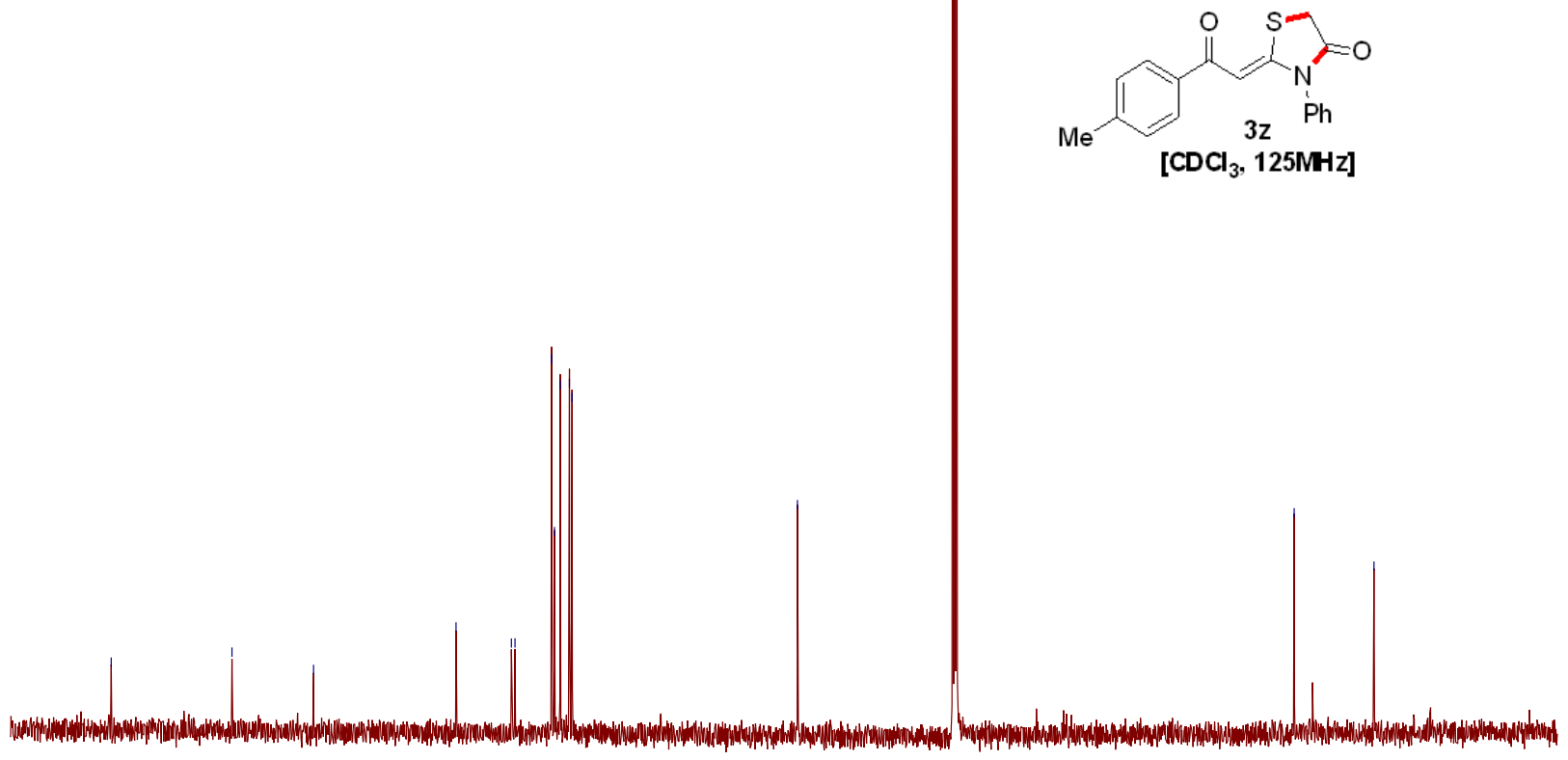

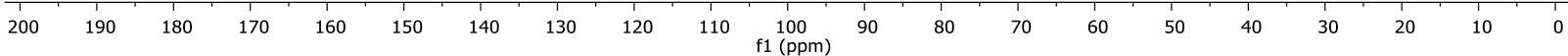


${ }^{1} \mathrm{H}$ and ${ }^{1} \mathrm{H}$ decoupled ${ }^{13} \mathrm{C}$ NMR spectra of compound 3nb

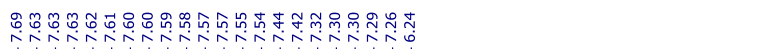
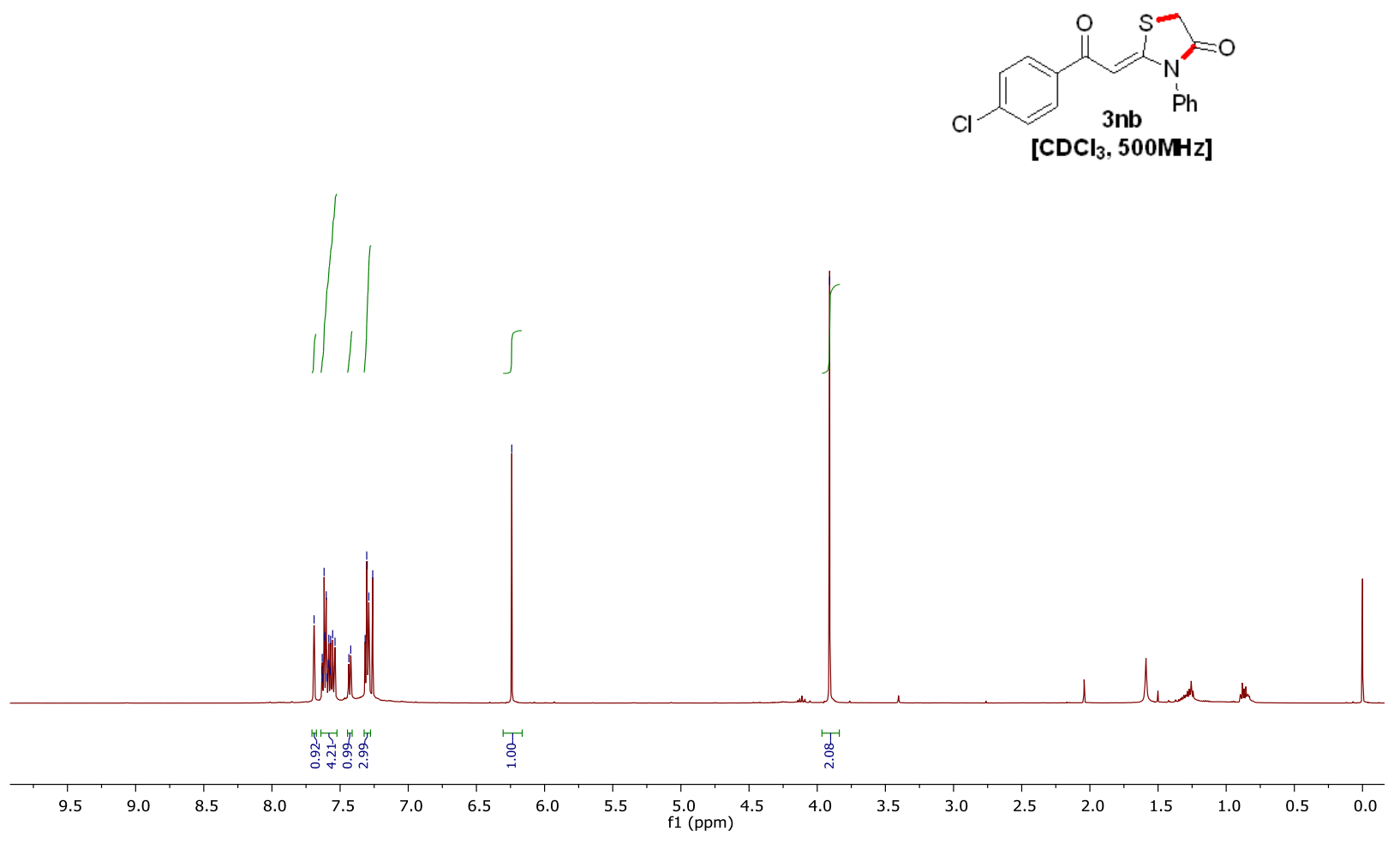

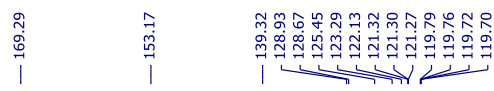

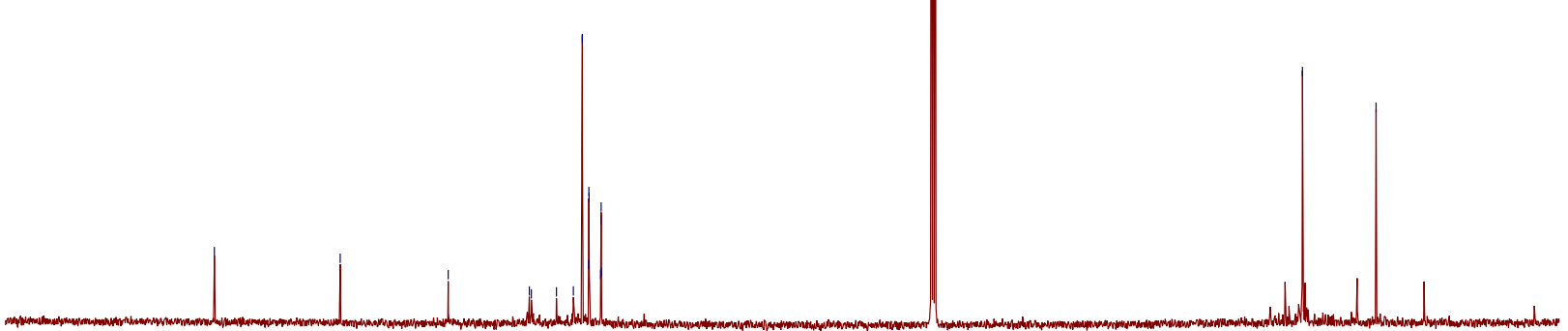

$170-160$

${ }_{\mathrm{f} 1(\mathrm{ppm})}^{100}$

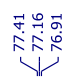

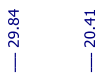

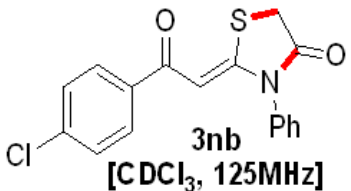

$\left[\mathrm{CDCl}_{3}, 125 \mathrm{MHz}\right]$ 
${ }^{1} \mathrm{H}$ and ${ }^{1} \mathrm{H}$ decoupled ${ }^{13} \mathrm{C}$ NMR spectra of compound $\mathbf{3 m b}$
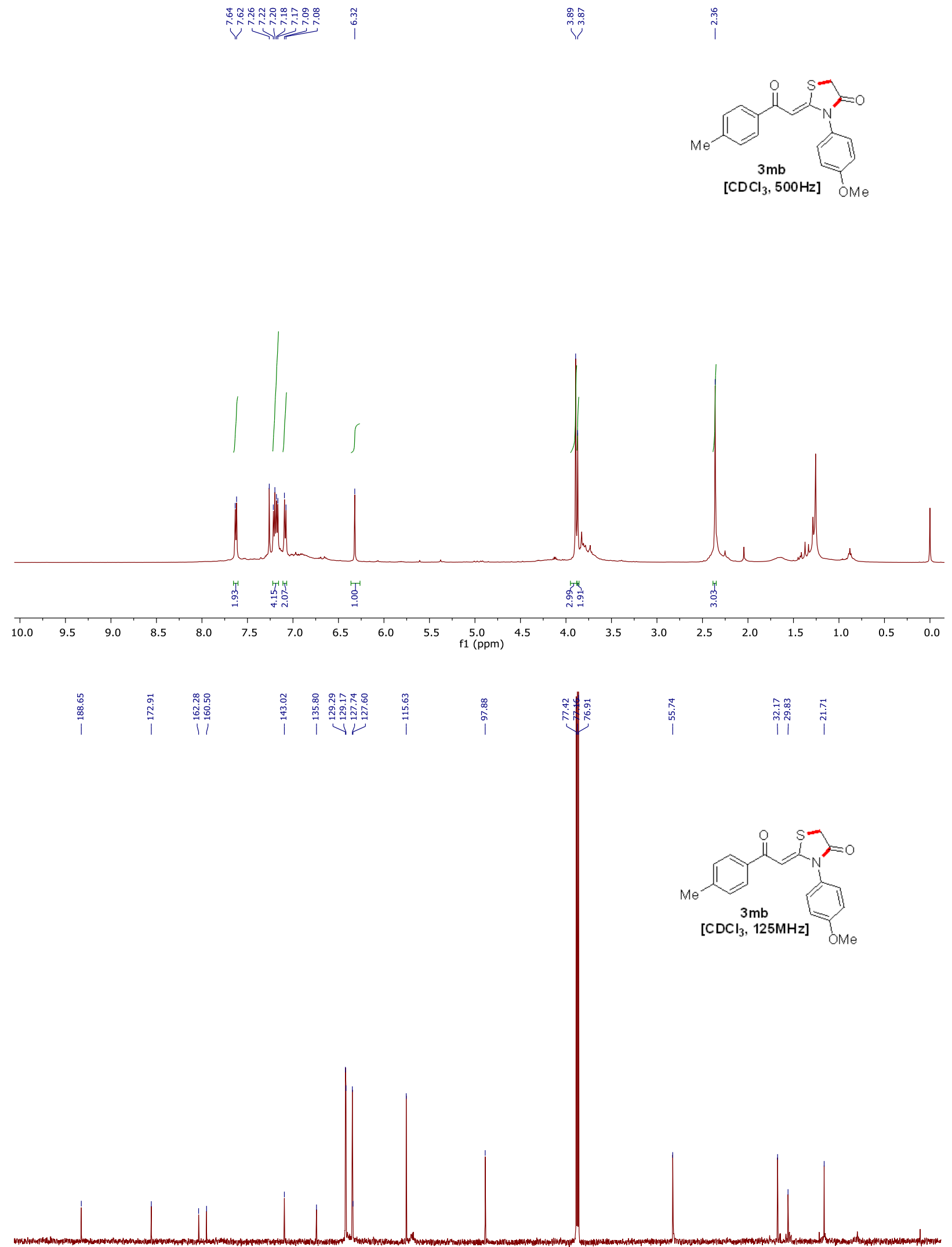

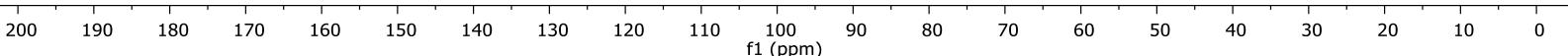


${ }^{1} \mathrm{H}$ and ${ }^{1} \mathrm{H}$ decoupled ${ }^{13} \mathrm{C}$ NMR spectra of compound 3eb

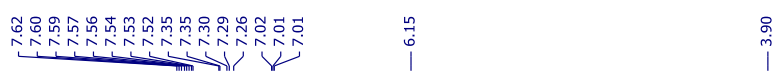

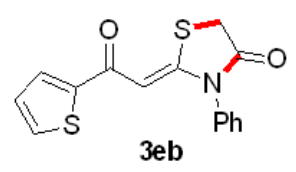

$\left[\mathrm{CDCl}_{3}, 500 \mathrm{MHz}\right]$

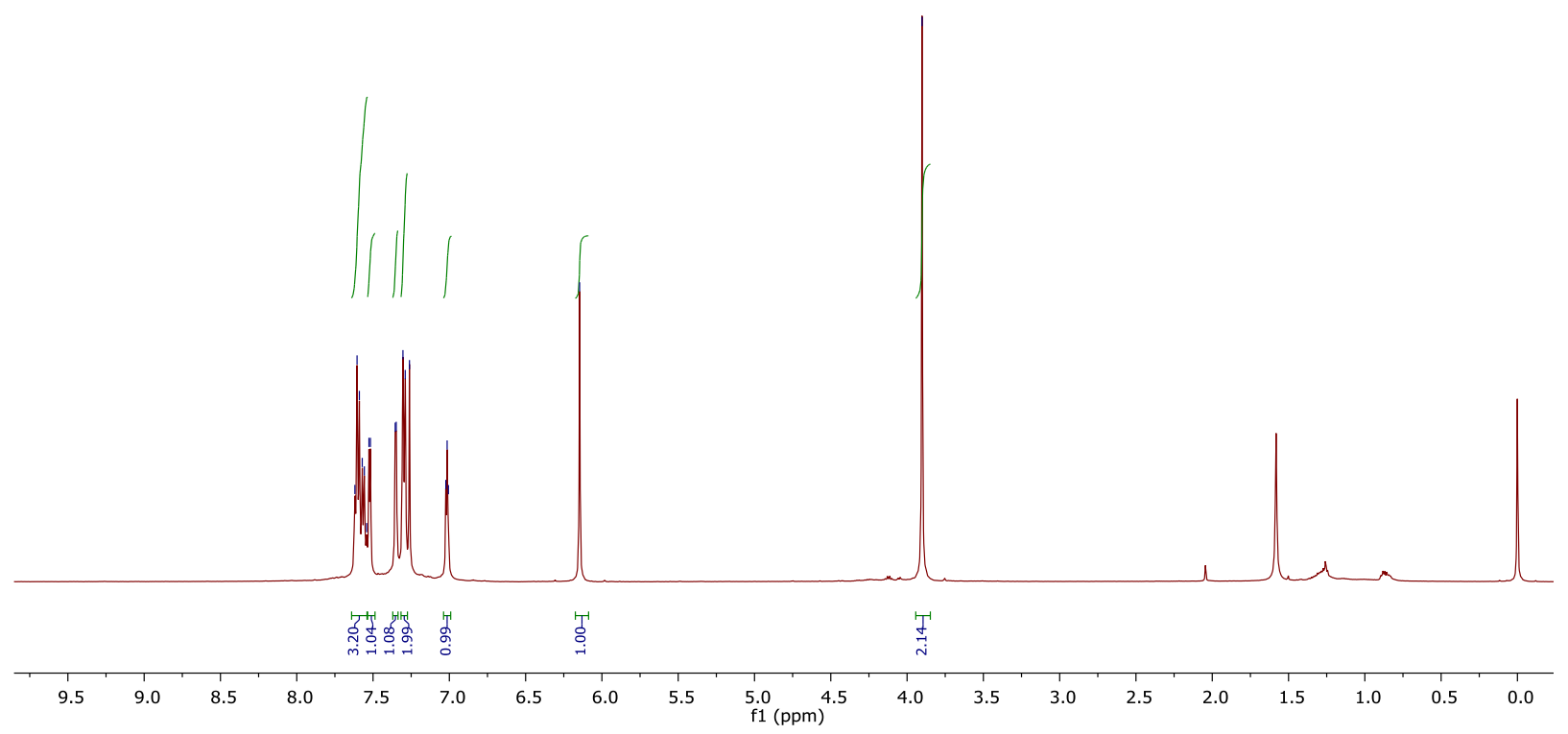

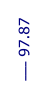

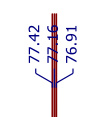

$\stackrel{\substack{n \\ \tilde{m}}}{1}$

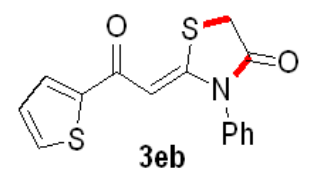

[CDCl $3,125 \mathrm{MHz}]$

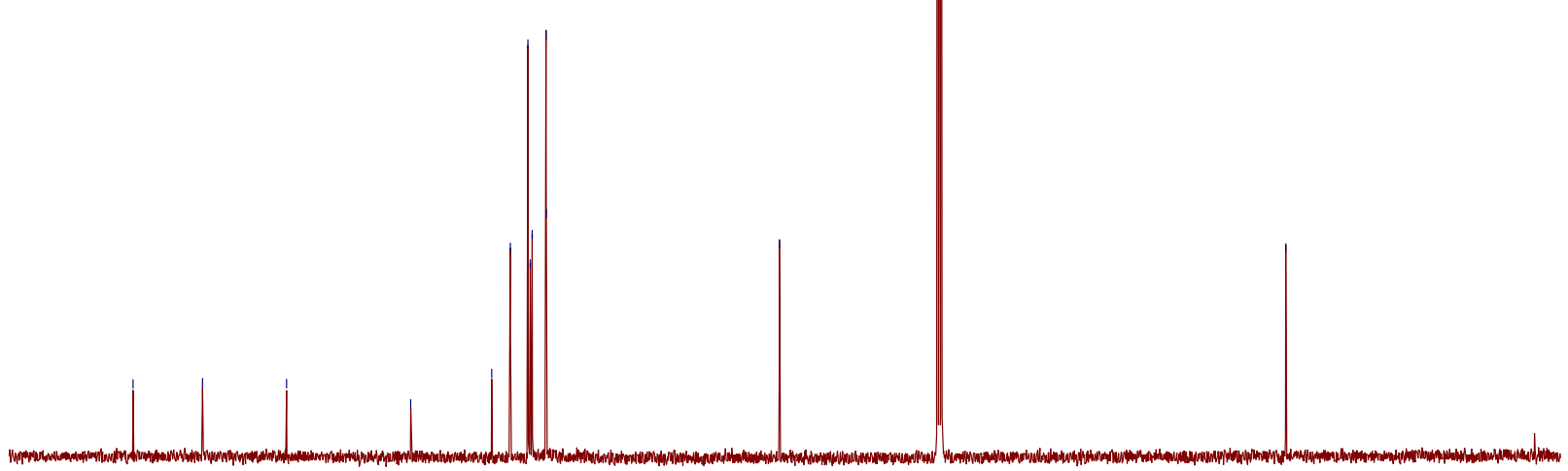

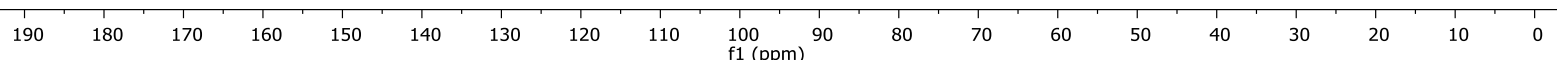


${ }^{1} \mathrm{H}$ and ${ }^{1} \mathrm{H}$ decoupled ${ }^{13} \mathrm{C}$ NMR spectra of compound 3ac

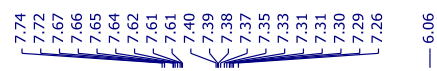
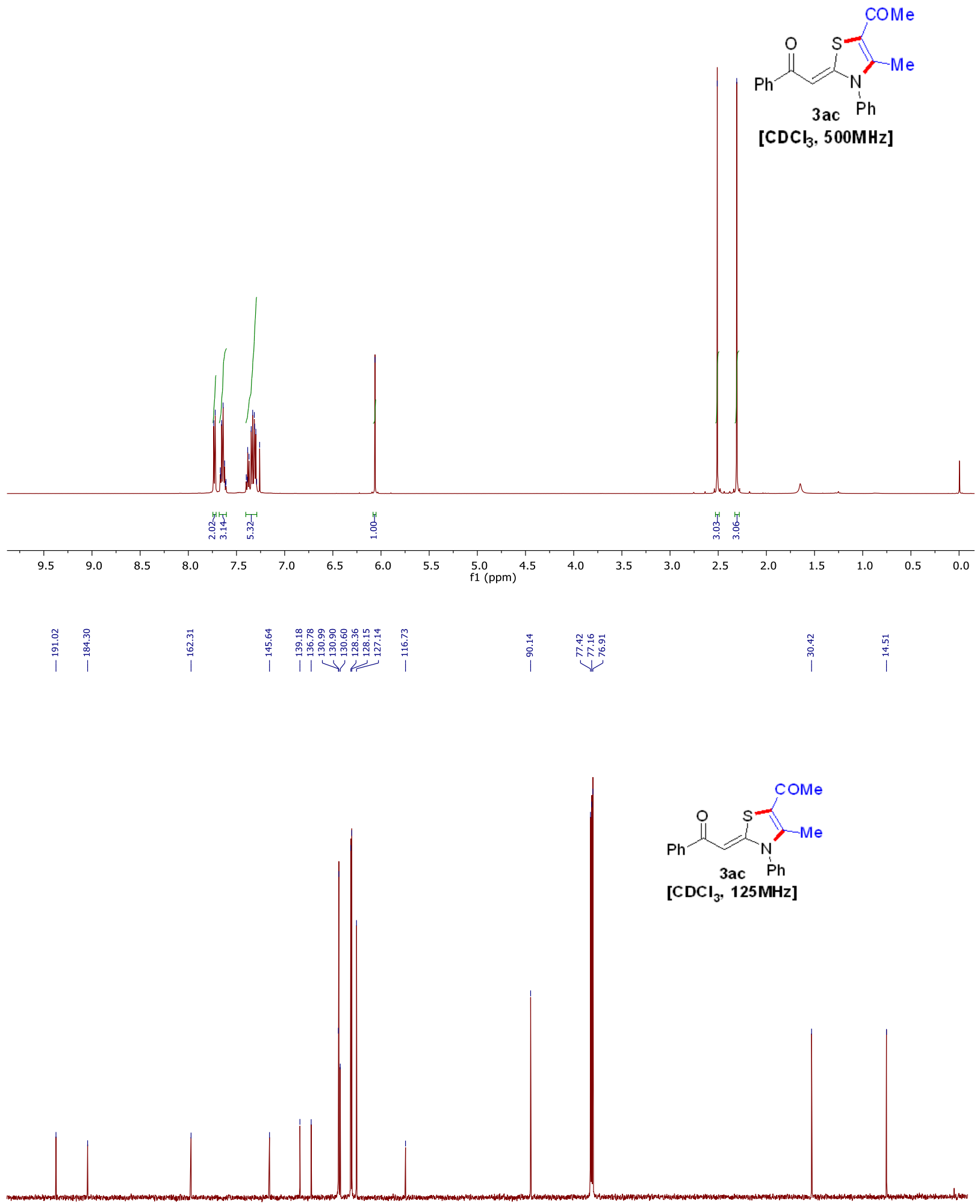

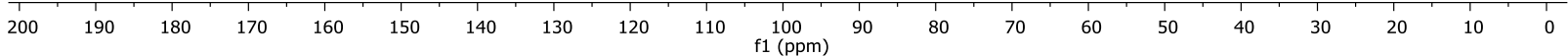


${ }^{1} \mathrm{H}$ and ${ }^{1} \mathrm{H}$ decoupled ${ }^{13} \mathrm{C}$ NMR spectra of compound 3tc

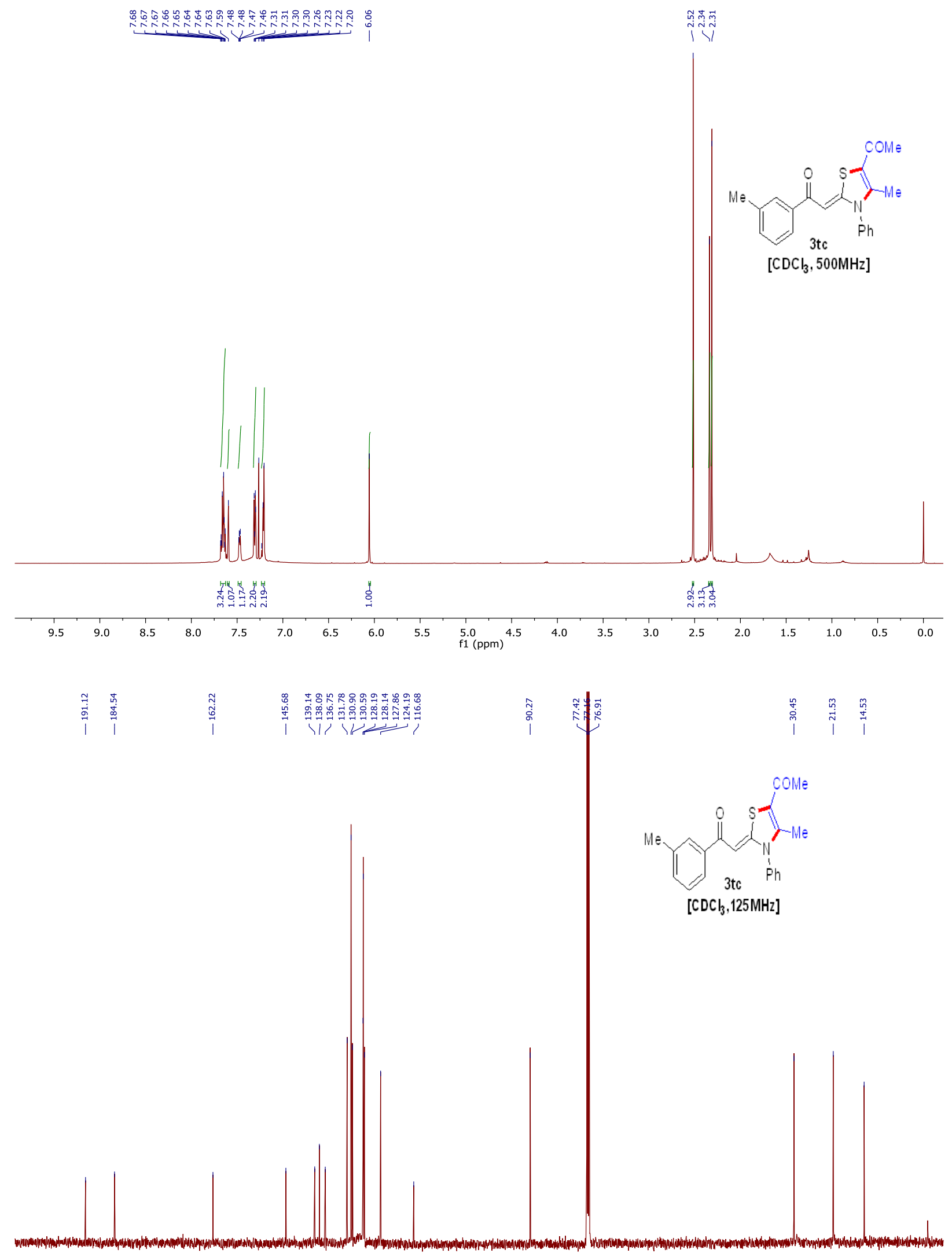

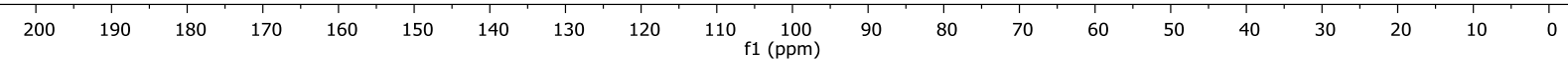


${ }^{1} \mathrm{H}$ and ${ }^{1} \mathrm{H}$ decoupled ${ }^{13} \mathrm{C}$ NMR spectra of compound $\mathbf{3 j c}$

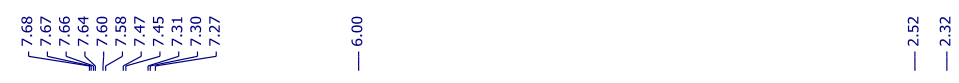

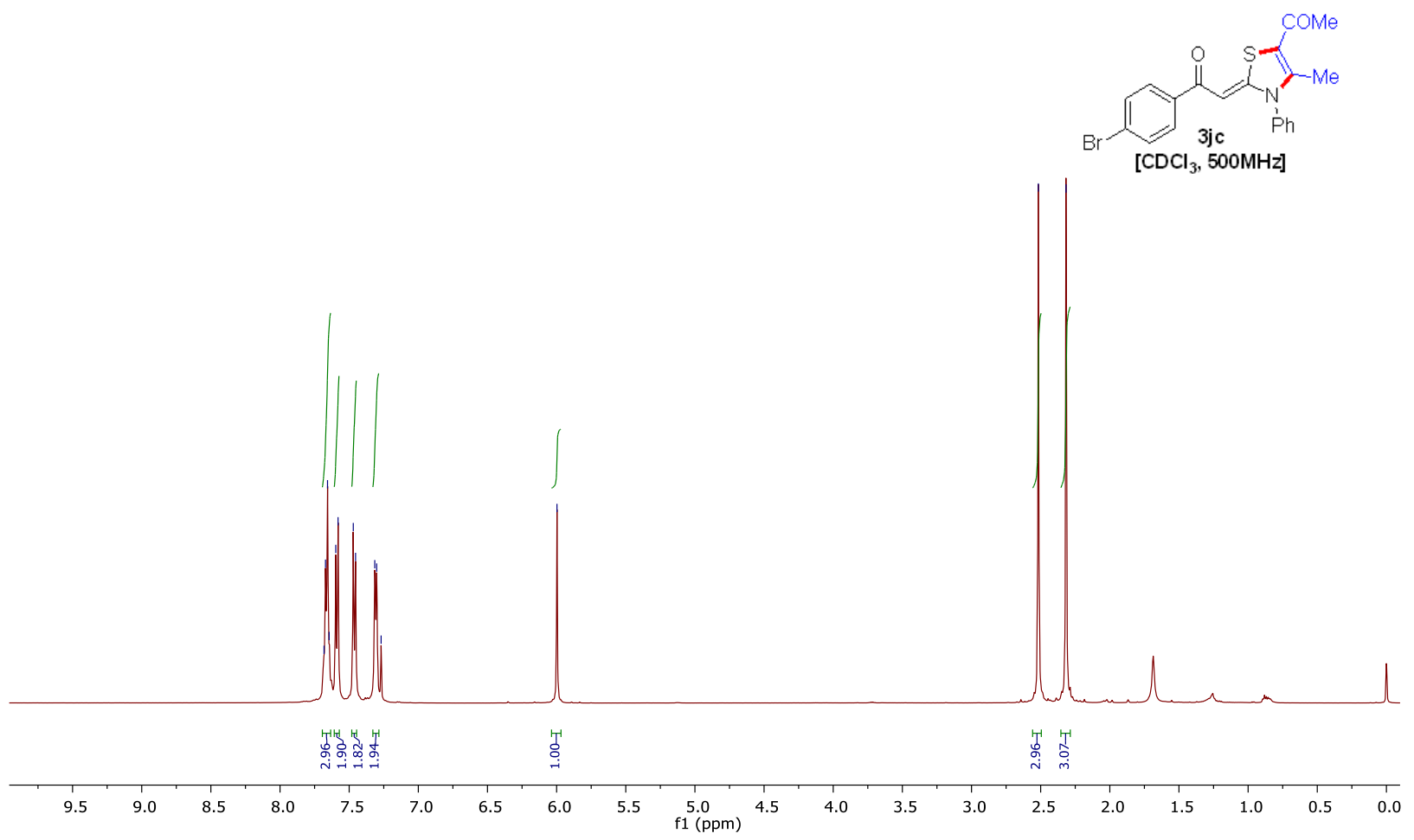

尊

|

咅
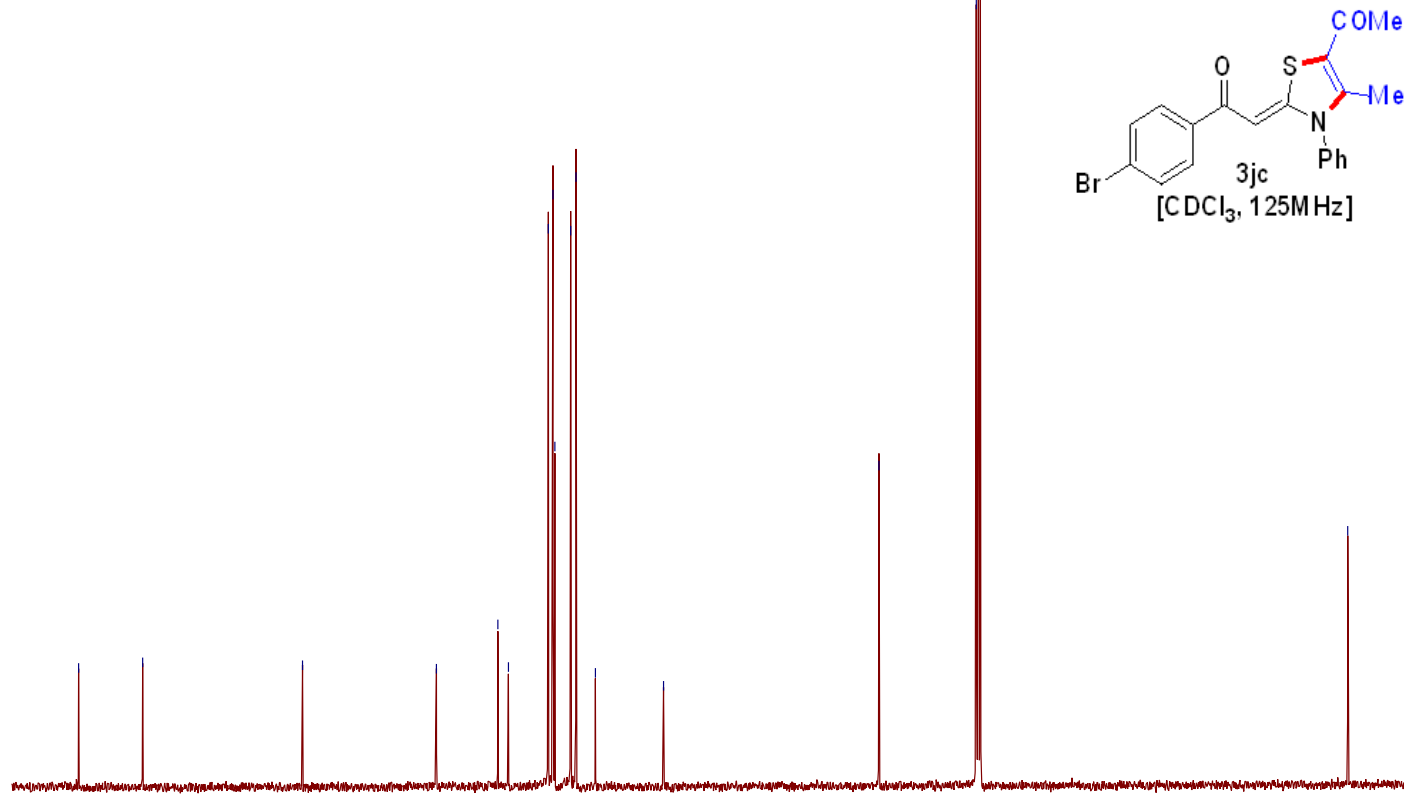

$\begin{array}{lllllll}190 & 180 & 170 & 160 & 150 & 140 & 130\end{array}$

$\begin{array}{llc}120 & 110 & 100 \\ & & 100\end{array}$

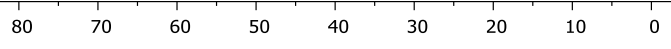


${ }^{1} \mathrm{H}$ and ${ }^{1} \mathrm{H}$ decoupled ${ }^{13} \mathrm{C}$ NMR spectra of compound $\mathbf{3 f c}$

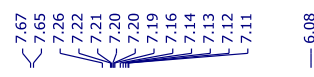

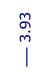
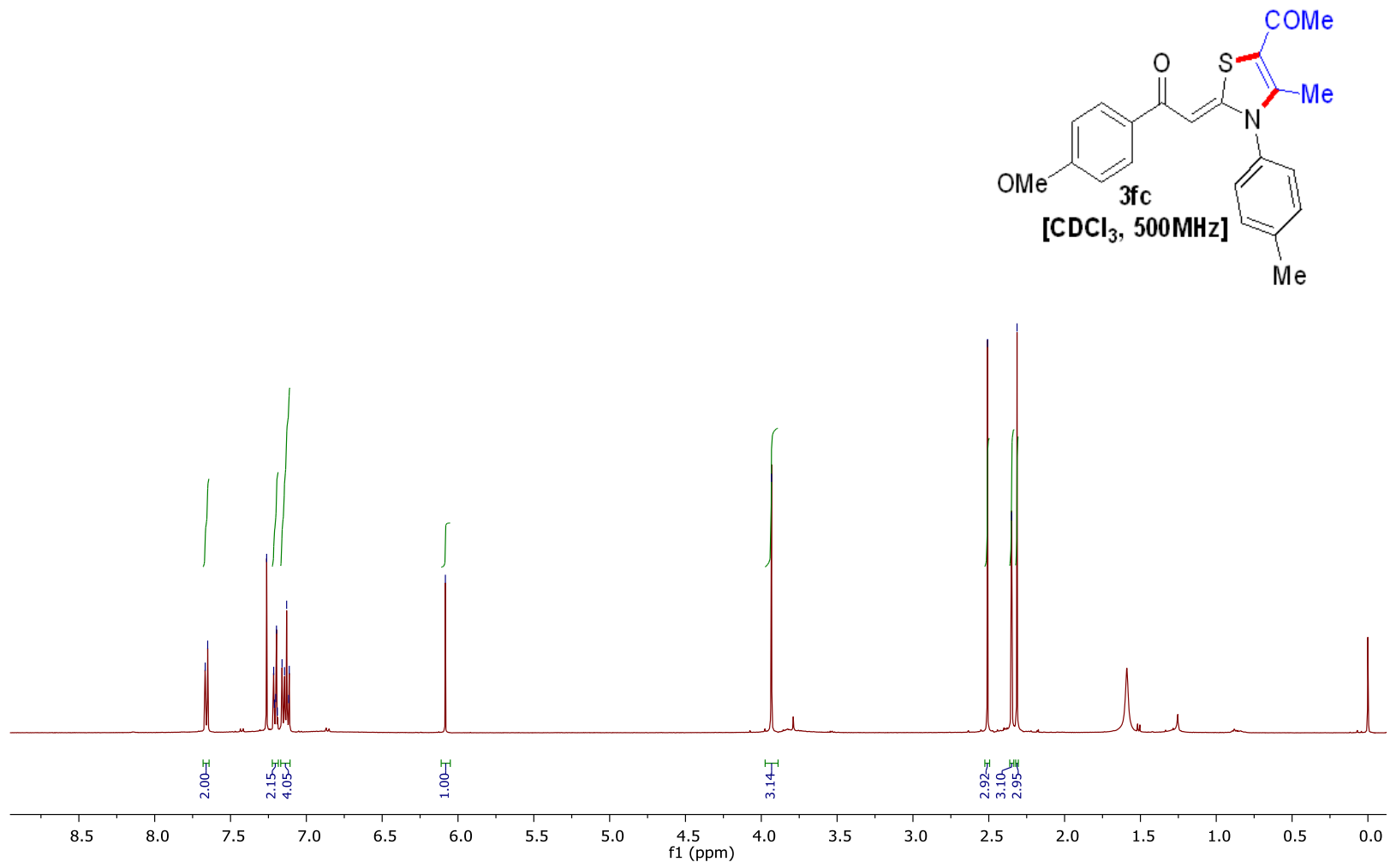

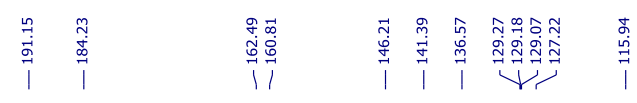
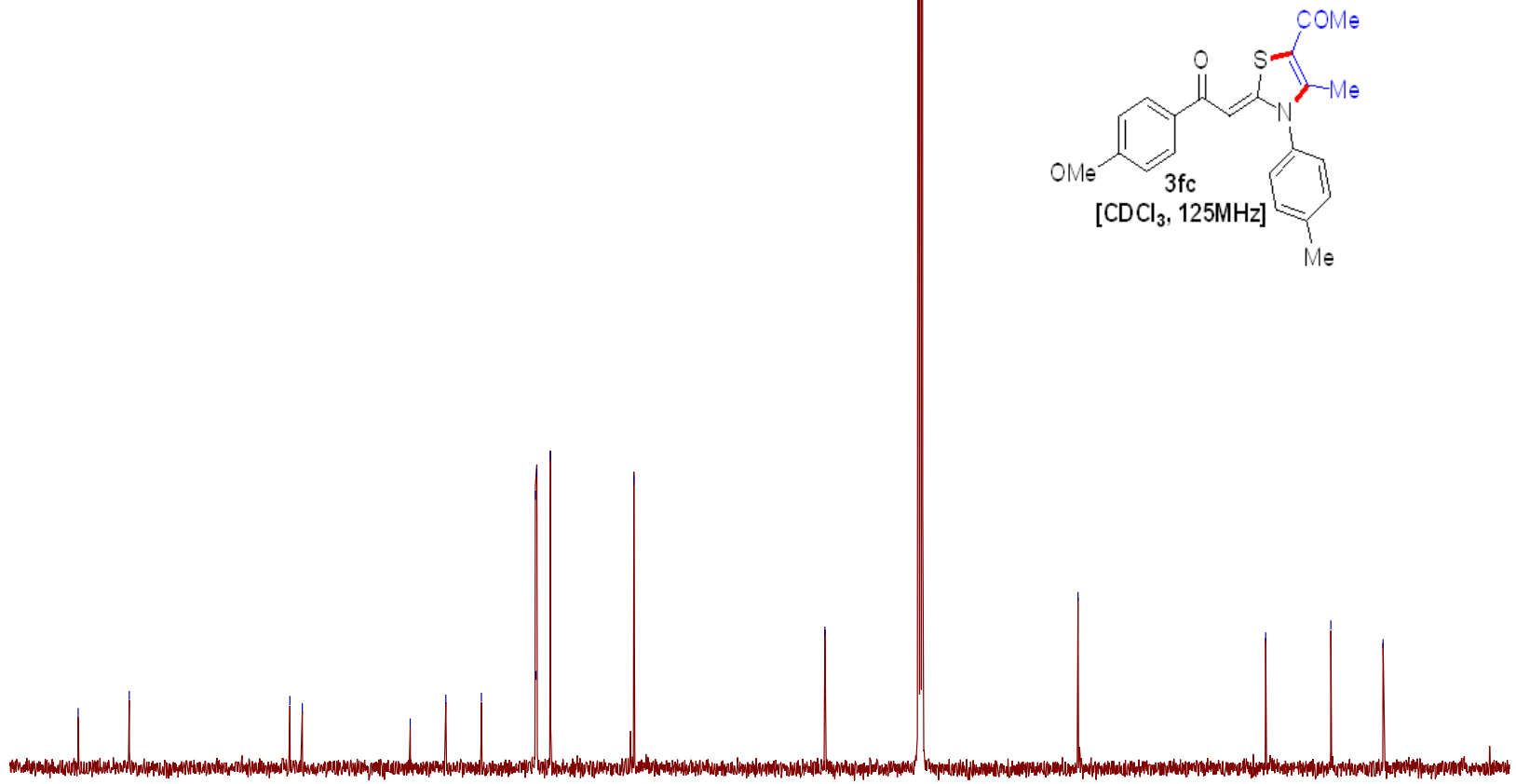

200 
${ }^{1} \mathrm{H}$ and ${ }^{1} \mathrm{H}$ decoupled ${ }^{13} \mathrm{C}$ NMR spectra of compound 3ec

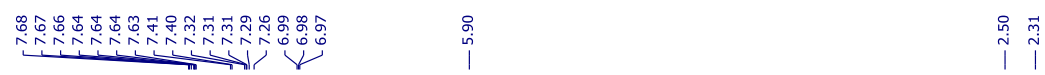

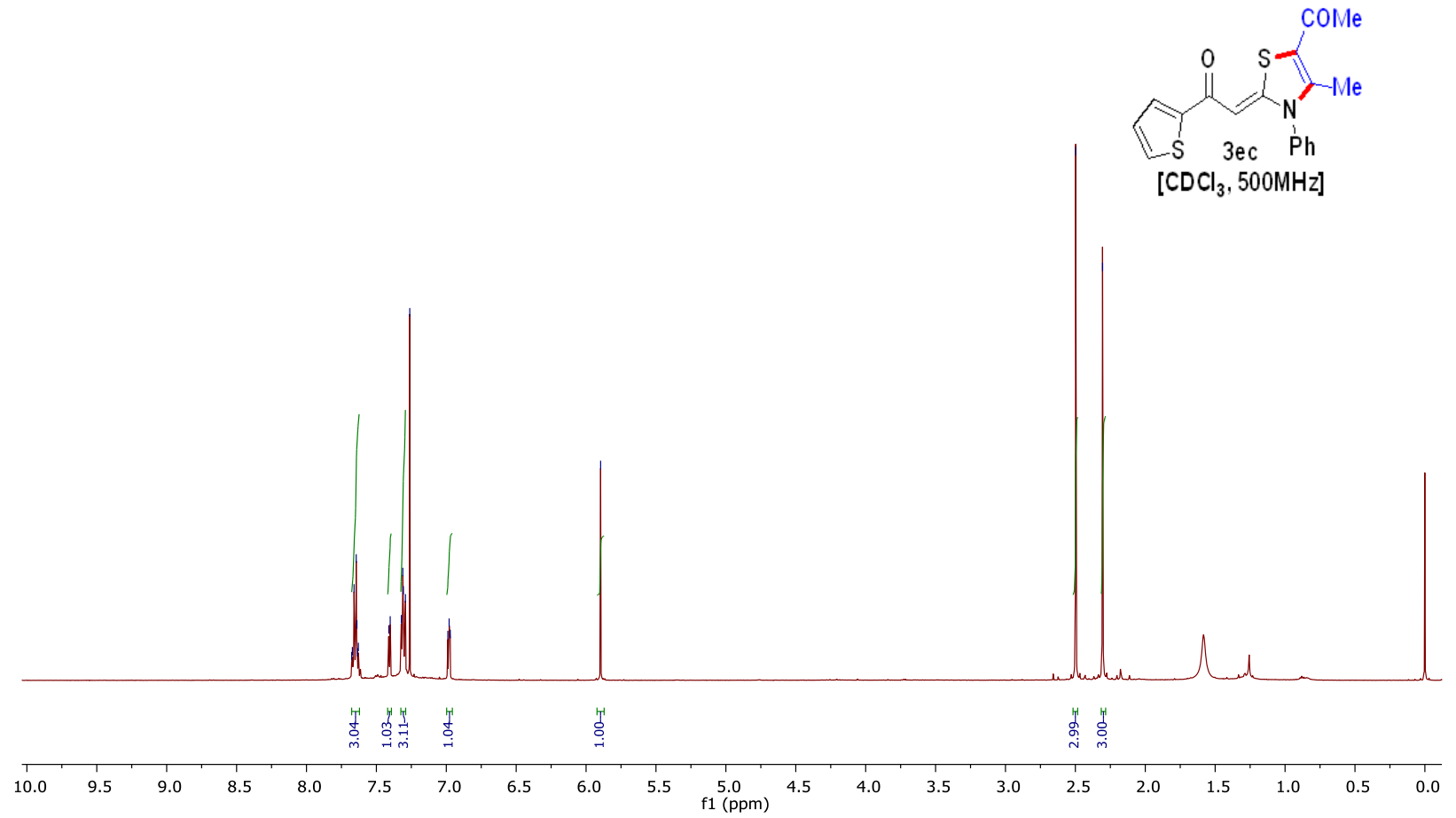

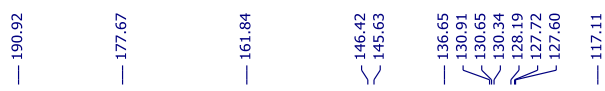

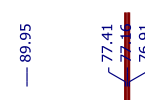

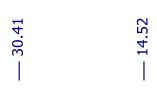

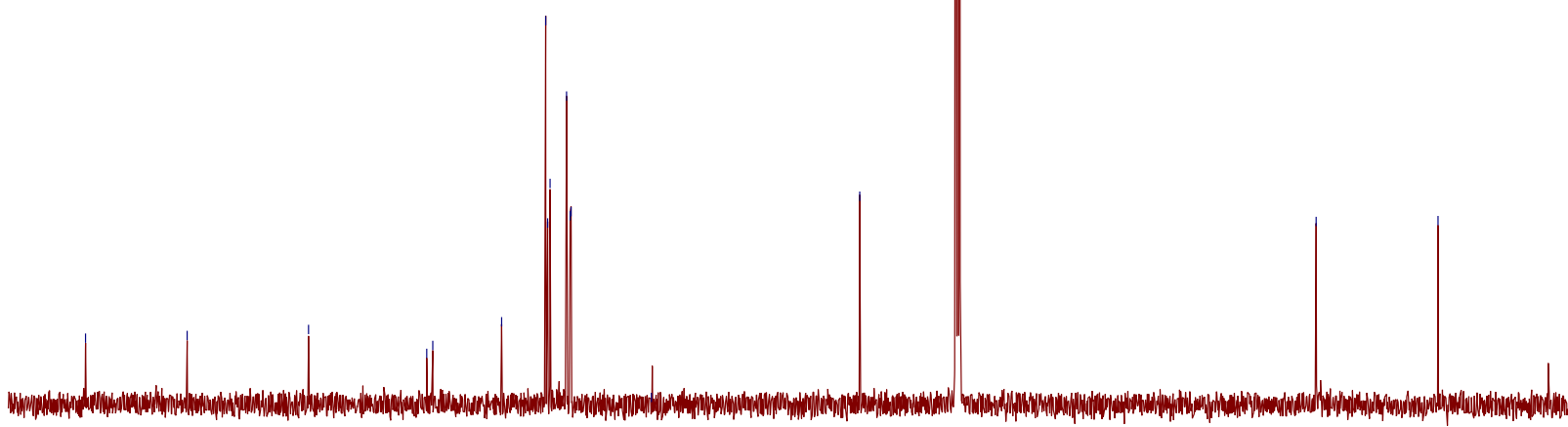

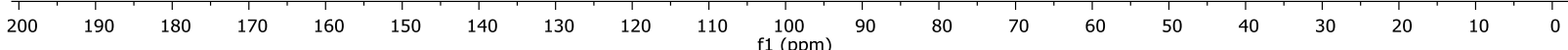


${ }^{1} \mathrm{H}$ and ${ }^{1} \mathrm{H}$ decoupled ${ }^{13} \mathrm{C}$ NMR spectra of compound $\mathbf{4 a}$

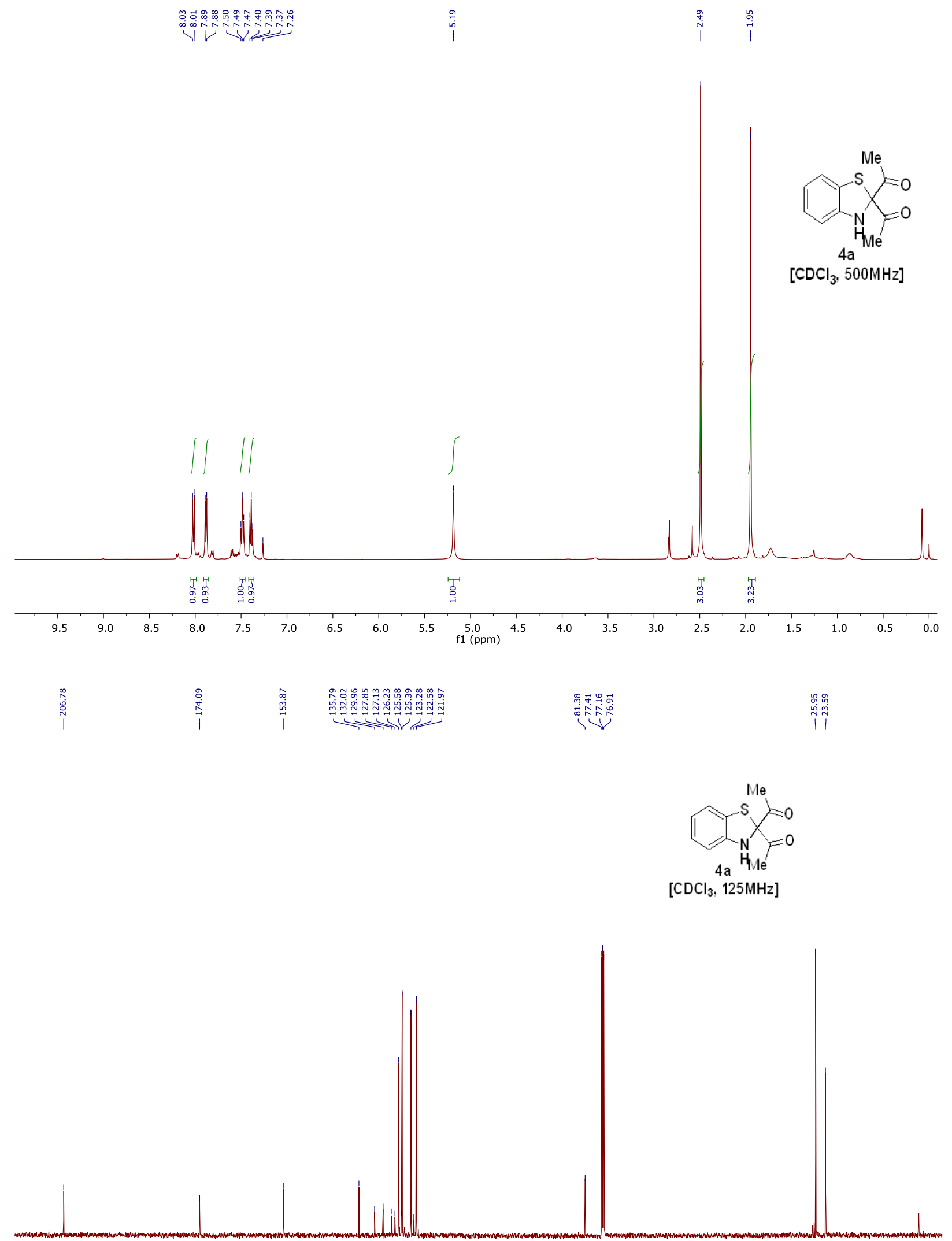

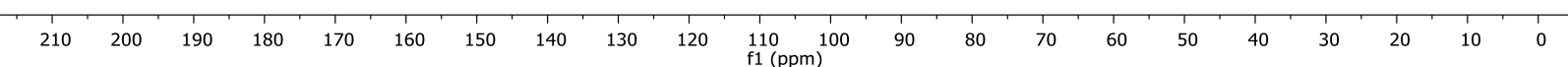


${ }^{1} \mathrm{H}$ and ${ }^{1} \mathrm{H}$ decoupled ${ }^{13} \mathrm{C}$ NMR spectra of compound $\mathbf{4 b}$

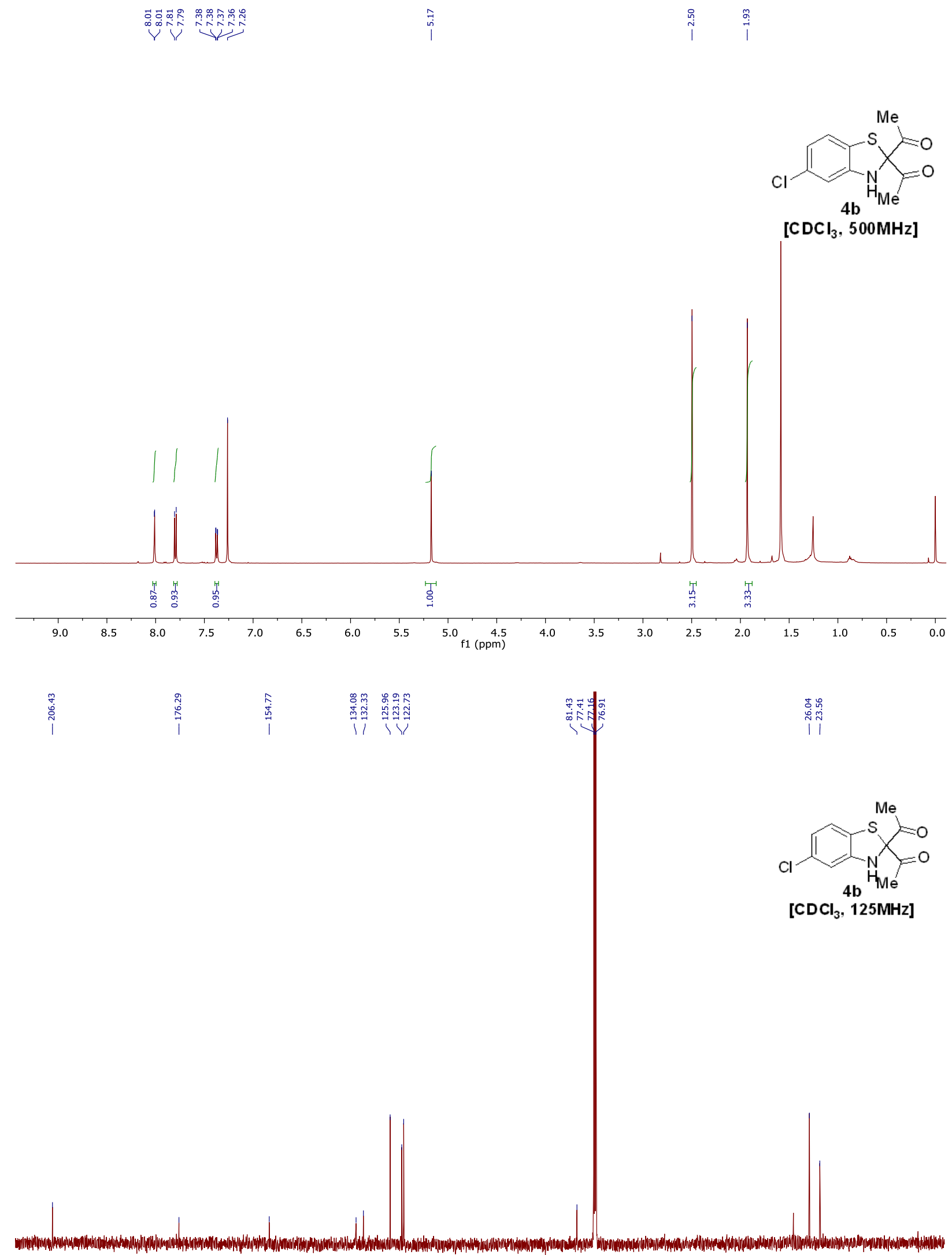

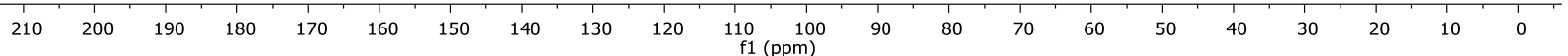




\section{Thermal ellipsoid plot and crystallization detail of compound $3 \mathrm{e}$}

The compound $\mathbf{3 e}$ after collecting from the column was dried under vacuum and dissolved in $4 \mathrm{~mL}$ of double distilled ethanol in a vial. Then the vial was kept at room temperature for slow evaporation. After ten days, a needle shape crystal formed in the vial, which was picked up and subjected to single crystal XRD study.

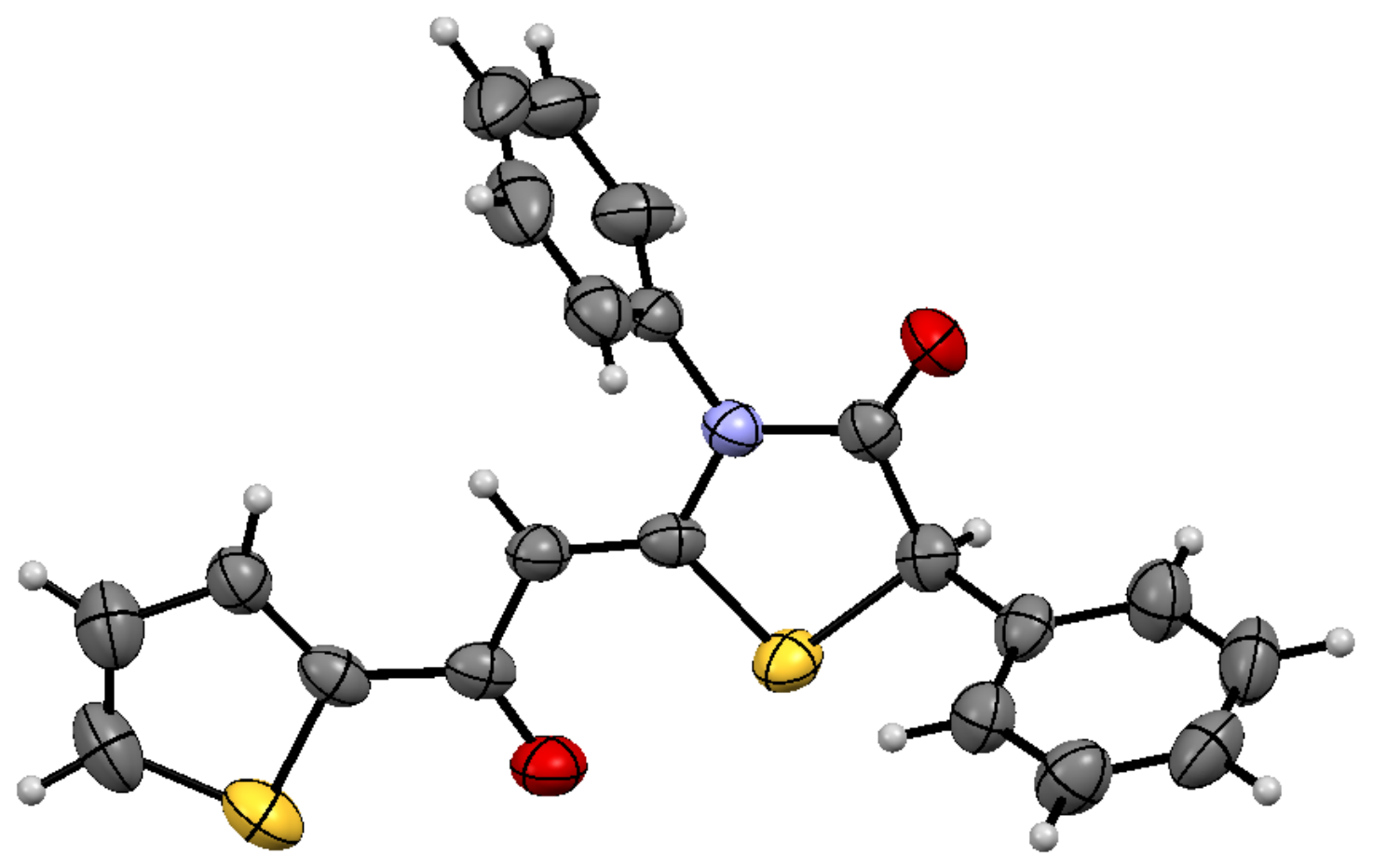

Figure S1. Thermal ellipsoids are plotted at the 50\% probability level 
ORTEP diagram of compound 3e (CCDC No 1977512)

\begin{tabular}{|c|c|c|c|}
\hline Bond precision: & $\mathrm{C}-\mathrm{C}=0.0038 \mathrm{~A}$ & Wavelengt $\mathrm{h}=0$ & $=0.71073$ \\
\hline Cell: & $\begin{array}{l}a=11.6074(6) \\
a l p h a=90\end{array}$ & $\begin{array}{l}\mathrm{b}=13.4571(7) \\
\text { beta }=114.599(1)\end{array}$ & $\begin{array}{l}\mathrm{c}=12.8460(7) \\
\text { gamma }=90\end{array}$ \\
\hline \multirow[t]{2}{*}{ Temperature: } & $298 \mathrm{~K}$ & & \\
\hline & Calculated & Reported & \\
\hline Volume & $1824.46(17)$ & $1824.46(17)$ & \\
\hline Space group & P $21 / n$ & P $21 / n$ & \\
\hline Hall group & $-\mathrm{P} 2 \mathrm{yn}$ & $-\mathrm{P} 2 \mathrm{yn}$ & \\
\hline Moiety formula & $\mathrm{C} 21 \mathrm{H} 15 \mathrm{~N}$ O2 $\mathrm{S} 2$ & C21 H15 N O & $\mathrm{O} 2 \mathrm{~S} 2$ \\
\hline Sum formula & $\mathrm{C} 21 \mathrm{H} 15 \mathrm{~N}$ O2 $\mathrm{S} 2$ & $\mathrm{C} 21 \mathrm{H} 15 \mathrm{~N} \mathrm{O}$ & $\mathrm{O} 2 \mathrm{~s} 2$ \\
\hline $\mathrm{Mr}$ & 377.46 & 377.46 & \\
\hline $\mathrm{Dx}, \mathrm{g} \quad \mathrm{cm}-3$ & 1.374 & 1.374 & \\
\hline z & 4 & 4 & \\
\hline $\mathrm{Mu} \quad(\mathrm{mm}-1)$ & 0.307 & 0.307 & \\
\hline F000 & 784.0 & 784.0 & \\
\hline F000' & 785.29 & & \\
\hline $\mathrm{h}, \mathrm{k}, \mathrm{lmax}$ & $15,17,16$ & $15,17,16$ & \\
\hline Nref & 4425 & 4417 & \\
\hline Tmin, Tmax & $0.912,0.912$ & $0.912,0.912$ & \\
\hline Tmin' & 0.912 & & \\
\hline
\end{tabular}

Correction method= \# Reported T Limits: Tmin=0.912 Tmax=0.912

$\mathrm{AbsCorr}=$ MULTI-SCAN

Data completeness $=0.998$

$\operatorname{Theta}(\max )=28.041$

$R($ reflections $)=0.0499(3028)$

$\mathrm{WR} 2($ reflections $)=0.1242(4417)$

$S=1.024$

Npar $=236$

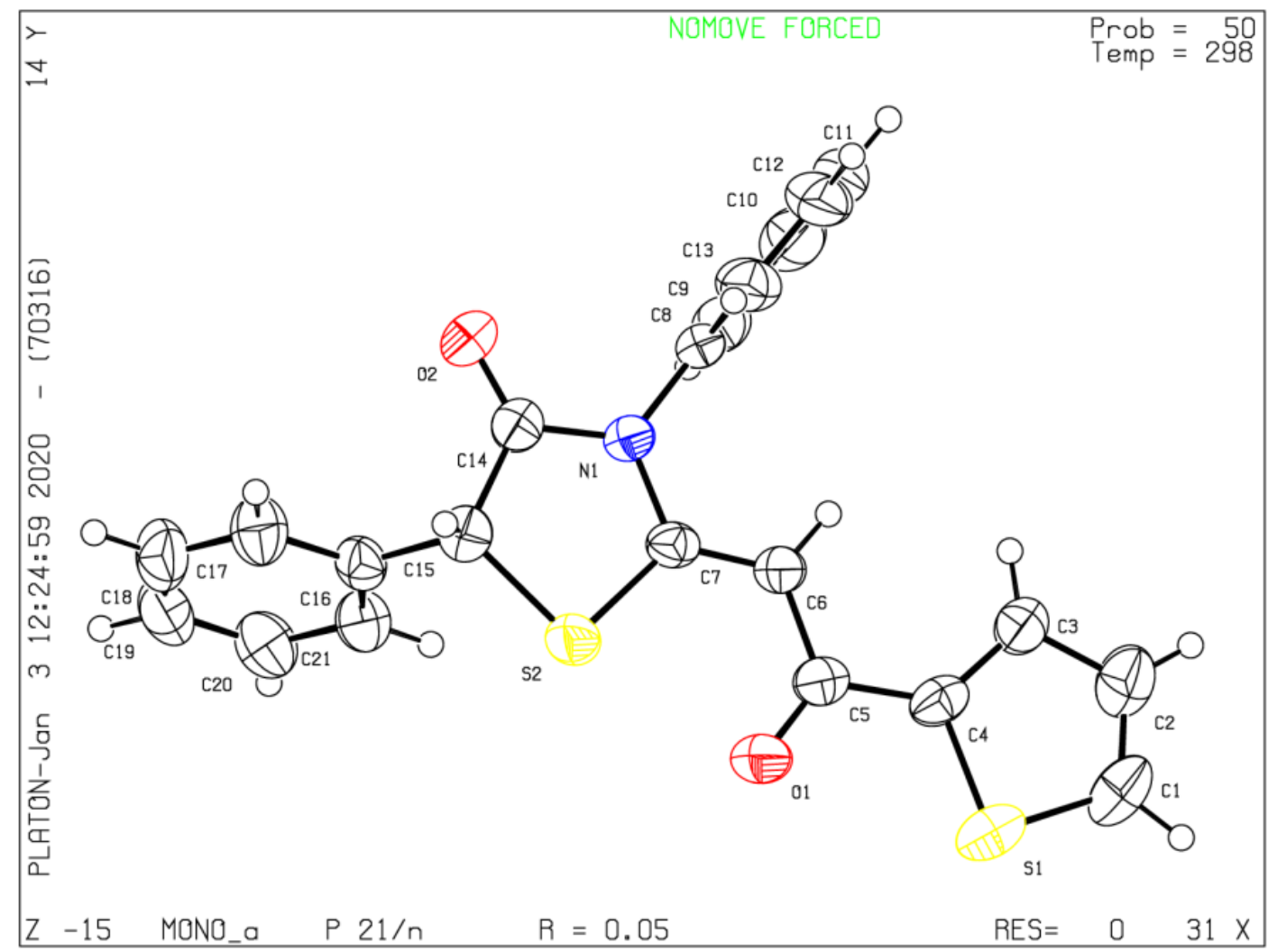




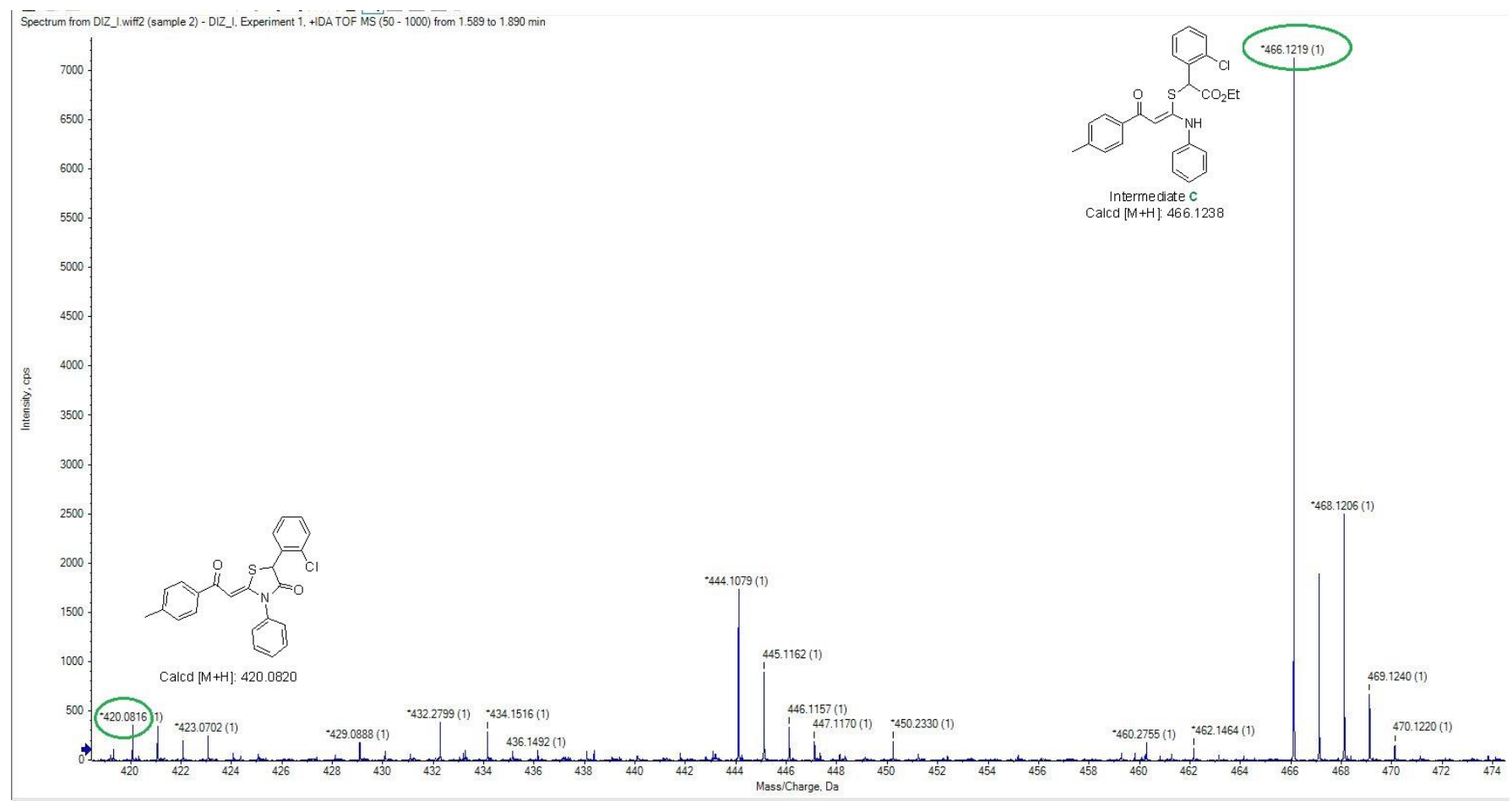

Figure S2: The positive-ion ESI mass spectrum of reaction mixture showing existence of $N, S$-acetal intermediate $\mathbf{C}$ 